\title{
Normal Approximation in LARGE NETWORK MODELS*
}

\author{
Michael P. Leung ${ }^{\dagger} \quad$ Hyungsik Roger Moon ${ }^{\ddagger}$
}

February 17, 2023

\begin{abstract}
We prove a central limit theorem for network moments in a model of network formation with strategic interactions and homophilous agents. Since data often consists of observations on a single large network, we consider an asymptotic framework in which the network size diverges. We argue that a modification of "exponential stabilization" conditions from the literature on geometric graphs provides a useful high-level formulation of weak dependence, which we use to establish an abstract central limit theorem. We then derive primitive conditions for stabilization using results in branching process theory. We discuss practical inference procedures justified by our results and outline a methodology for deriving primitive conditions that can be applied more broadly to other large network models with strategic interactions.
\end{abstract}

JEL Codes: C31, C57, D85

KEYWORDS: social networks, strategic interactions, weak dependence, network formation

${ }^{*}$ We thank the editor and referees for suggestions that helped improve the exposition of the paper. We thank seminar audiences at OSU, UBC, UC Berkeley, Yale, the 2018 Turing workshop on the Statistics of Network Analysis, and the 2018 Shanghai Workshop of Econometrics. Leung and Moon gratefully acknowledge financial support from the National Science Foundation under grants SES-1755100 and SES-1625586, respectively.

${ }^{\dagger}$ Department of Economics, UC Santa Cruz. E-mail: leungm@ucsc.edu.

${ }^{\ddagger}$ Department of Economics, University of Southern California and Yonsei University. E-mail: moonr@usc.edu. 


\section{LEUNG AND MOON}

\section{Introduction}

Network models have attracted considerable attention in economics as tractable representations of non-market interactions, such as peer effects and social learning, and economic relationships, such as financial and trade networks. The economic perspective on networks emphasizes the importance of strategic interactions or externalities (Jackson et al., 2021). One strand of the literature studies social interactions, how an individual's behavior interacts with those of her social contacts. A second strand studies network formation, why an individual chooses particular social contacts in the first place, and how her choices affect those of others. In both cases, externalities create a wedge between choices that are optimal from the individual's perspective and those that are efficient for society, which has important consequences for policymaking. For instance, they motivate associational redistribution, policies that intervene on the network structure (Durlauf, 1996). However, when preferences are misaligned with the policy objective, the endogenous response to the intervention may diverge from the intended outcome (Carrell et al., 2013). It is therefore of interest to develop econometric methodologies for measuring network externalities.

The challenge is that network data typically consists of observations on a single large network, and strategic interactions induce a non-standard form of cross-sectional dependence between observations. A large-sample theory requires conditions under which the amount of "independent information" grows with the number of nodes or agents in the network, despite network dependence.

The main contribution of this paper is a central limit theorem (CLT) for a model of strategic network formation. We discuss practical inference procedures justified by the result and also provide a method of proof that can be applied more broadly to other network models. ${ }^{1}$ Our CLT applies to a large class of network moments computed from a single large network, which are averages of node-level statistics $n^{-1} \sum_{i=1}^{n} \psi_{i}$, where $\psi_{i}$ is some function of the network (or network time series) and node types. A simple example is the degree of node $i$, which is the number of links involving $i$. More generally, the class of moments includes the average clustering coefficient, regression estimators, and subnetwork counts, the latter of which is useful for inference in econometric models of network formation (Sheng, 2020).

\footnotetext{
${ }^{1} \mathrm{~A}$ previous version of the paper also considers applications to dynamic network formation (Leung and Moon, 2019), and a subsequent paper by Leung (2019a) applies the methodology to discrete games on networks.
} 


\section{Normal Approximation in Large Network Models}

Such models are useful to understand the incentives for developing social connections, for example the formation of risk-sharing networks in the rural Philippines (Fafchamps and Gubert, 2007) and research partnerships in the biotechnology industry (Powell et al., 2005). They can be used to distinguish between different mechanisms for link formation proposed in the theory literature, including preferential attachment (Barabási and Albert, 1999), strategic transitivity (Mele, 2022; Ridder and Sheng, 2022), and homophily (McPherson et al., 2001). Finally, these models are useful for forecasting the effects of counterfactual interventions (Mele, 2020) and accounting for dependence between the network and unobservables of a social interactions model (Badev, 2013; Goldsmith-Pinkham and Imbens, 2013).

We emphasize two main technical contributions. The first is an abstract CLT that holds under high-level conditions, which is an extension of limit theorems from the literature on geometric graphs. The key condition is a modification of "stabilization" assumptions from that literature that provides a useful formulation of weak dependence for our purposes. Stabilization essentially requires $\psi_{i}$ to only be a function of a random subset of nodes whose size has exponential tails. In our applications, this random subset is generally a union of network components, which are difficult combinatorial objects to analyze. Our primary contribution is to develop a methodology and set of useful lemmas for deriving tail bounds for complex objects of this type. We show how the methodology can be used to derive primitive sufficient conditions for stabilization in models of strategic network formation.

The basic idea behind the methodology is that, in the presence of strategic interactions, the pairwise stability of a link can depend on the existence of "neighboring" links, which in turn can depend on other links, and so on. The longer the lengths of these chains of dependent links, the stronger the extent of autocorrelation. We adopt a well-known technique in random graph theory and bound the lengths of these chains by subcritical branching processes whose sizes we prove to have exponential tails. Primitive conditions for subcriticality can be interpreted as weak-dependence conditions that restrict the strength of strategic interactions, analogous to conditions imposed on spatial and temporal autoregressive models that bound the magnitude of the autoregressive parameter below one.

A growing literature studies frequentist inference in network formation models when the econometrician observes a single network. Leung (2019b) and Menzel (2017) develop laws of large numbers for models of strategic network formation. The former 


\section{LEUNG AND MOON}

paper modifies a weaker stabilization condition due to Penrose and Yukich (2003) and uses branching processes to derive primitive sufficient conditions. Our paper tackles the more difficult problem of obtaining a normal approximation, which naturally requires a stronger stabilization condition, and discusses practical inference procedures newly justified by the result. We prove the CLT by extending results in Penrose and Yukich (2005) and Penrose (2007); a more detailed discussion of our contributions relative to this literature can be found in $§ 3.3$. Kuersteiner (2019) takes a different approach, using a novel conditional mixingale type assumption defined in terms of a random metric of distance.

Leung (2015b) and Ridder and Sheng (2022) study strategic network formation under incomplete information. In this setting, links are independent conditional on observables, whereas the model we study can be microfounded as games of complete information and allow for unobserved heterogeneity, which generates dependence between potential links even conditional on observables.

Charbonneau (2017), Dzemski (2019), Graham (2017), and Jochmans (2018) consider dyadic link formation models without strategic interactions but allow for nodelevel fixed effects. A large statistics literature studies models without strategic interactions, for example stochastic block models (Bickel et al., 2011) and latent-space models (Hoff et al., 2002). These are useful for their parsimony and tasks such as community detection. Chandrasekhar and Jackson (2021) and Boucher and Mourifié (2017) study other statistical models allowing for interdependence between links.

The next section presents a model of strategic network formation and defines network moments. In $\S 3$, we state high-level conditions for a CLT and outline its proof. For readers interested in applying a CLT for network formation, we suggest skipping $\S 3$ and reading $\S 4$, which provides primitive sufficient conditions for the key high-level weak-dependence condition in $\S 3$. It also outlines a general methodology for verifying the high-level condition that can be applied to other network models. In $\S 5$, we discuss practical inference procedures, and $\S 6$ concludes. All proofs are given in the appendix and supplemental appendix.

We introduce standard notation and terminology for networks. We represent a network on a set of $n$ nodes an $n \times n$ adjacency matrix, where the $i j$ th entry $A_{i j}$, termed the potential link, is an indicator for whether nodes $i, j$ are connected. Following the usual convention, we require that $A_{i i}=0$ for all nodes $i$, meaning that there are no self-links. We focus on undirected networks, so $A_{i j}=A_{j i}$. For two 


\section{Normal Approximation in Large Network Models}

networks $\boldsymbol{A}, \boldsymbol{A}^{\prime}$, we say that $\boldsymbol{A}$ is a subnetwork of $\boldsymbol{A}^{\prime}$ if every link in $\boldsymbol{A}$ is a link in $\boldsymbol{A}^{\prime}$. A path in a network from node $i$ to $j$ is a sequence of distinct nodes starting with $i$ and ending with $j$ such that for each consecutive node pair $k, k^{\prime}$ in this sequence, $A_{k k^{\prime}}=1$. Its length is the number of links it involves. The path distance between two nodes $i, j$ in $\boldsymbol{A}$ is the length of the shortest path that connects them if a path exists and $\infty$ otherwise. The $K$-neighborhood of a node $i$ in $\boldsymbol{A}$, denoted by $\mathcal{N}_{\boldsymbol{A}}(i, K)$, is the set of all nodes $j$ of path distance at most $K$ from $i$ (including $i$ ). Finally the component of a node $i$ with respect to a network $\boldsymbol{A}$ is the set of all nodes $j$ at finite path distance from $i$.

\section{Model}

Let $\mathcal{N}_{n}=\{1, \ldots, n\}$ be a set of nodes, where each $i \in \mathcal{N}_{n}$ is endowed with a type $\left(X_{i}, Z_{i}\right)$. We distinguish $X_{i} \in \mathbb{R}^{d}$ as the position of node $i$, a continuously distributed vector of homophilous (defined below) attributes with density $f$. Each node pair $(i, j)$ is endowed with a real-valued random utility shock $\zeta_{i j}=\zeta_{j i}$. Let $\left\{\left(X_{i}, Z_{i}\right)\right\}_{i \in \mathbb{N}}$ and $\left\{\zeta_{i j}: i \in \mathcal{N}_{n}, j>i\right\}$ both be i.i.d. and independent.

The observed network $\boldsymbol{A}$ satisfies

$$
A_{i j}=\mathbf{1}\left\{V\left(r_{n}^{-1}\left\|X_{i}-X_{j}\right\|, S_{i j}, Z_{i}, Z_{j}, \zeta_{i j}\right)>0\right\}
$$

where $V(\cdot)$ is an $\mathbb{R}$-valued function, $\|\cdot\|$ a norm on $\mathbb{R}^{d}, r_{n}$ a positive constant that will control the network's sparsity, and $S_{i j}$ a vector of statistics that can depend on $\boldsymbol{A}$ and captures strategic interactions. If we interpret $V(\cdot)$ as the joint surplus agents $i$ and $j$ enjoy from forming a link, then (1) corresponds to the well-known pairwise-stability solution concept under transferable utility (Jackson, 2010).

Example 1. Consider the linear latent index

$$
V\left(r_{n}^{-1}\left\|X_{i}-X_{j}\right\|, S_{i j}, Z_{i}, Z_{j}, \zeta_{i j}\right)=\left(S_{i j}, Z_{i}, Z_{j}\right)^{\prime} \theta-r_{n}^{-1}\left\|X_{i}-X_{j}\right\|+\zeta_{i j}
$$

and a simple example of $S_{i j}$

$$
S_{i j}=\max _{k} A_{i k} A_{j k}
$$

The latter is an indicator for whether $i$ and $j$ share a common friend (used for example 


\section{LEUNG AND MOON}

by Menzel, 2017). If the coefficient of $S_{i j}$ is positive, then node pairs $(i, j)$ sharing a common neighbor $\left(S_{i j}=1\right)$ are more likely to form a link. This rationalizes the well-known stylized fact that networks are commonly highly clustered, meaning that nodes with common neighbors are themselves typically neighbors (Jackson, 2010).

Example 2. Sheng (2020) studies the specification

$$
\begin{aligned}
V\left(r_{n}^{-1}\left\|X_{i}-X_{j}\right\|,\right. & \left.S_{i j}, Z_{i}, Z_{j}, \zeta_{i j}\right)=\beta_{0}+\left(Z_{i}+Z_{j}\right)^{\prime} \beta_{1} \\
& +\beta_{2} r_{n}^{-1}\left\|X_{i}-X_{j}\right\|+\gamma_{1} \sum_{k=1}^{n}\left(A_{i k}+A_{j k}\right)+\gamma_{2} \sum_{k=1}^{n} A_{i k} A_{j k}+\zeta_{i j}
\end{aligned}
$$

This also corresponds to a linear latent index but with

$$
S_{i j}=\left(\sum_{k=1}^{n} A_{i k} A_{j k}, \sum_{k=1}^{n} A_{i k}, \sum_{k=1}^{n} A_{j k}\right) .
$$

The first component of (4) plays a role analogous to (2), being a count of the number of common neighbors. The second and third components are respectively the degrees (number of neighbors) of $i$ and $j$. If $\gamma_{1}>0$, this captures a popularity effect.

It should be noted that the assumptions we impose for a CLT require $S_{i j}$ to be uniformly bounded, and existing work on large-sample theory for network formation shares this limitation (Boucher and Mourifié, 2017; Menzel, 2017; Ridder and Sheng, 2022). Statistics such as (4) may be modified to satisfy uniform boundedness by truncation, for example $\min \left\{\sum_{k=1}^{n} A_{i k}, \Delta\right\}$ for some user-specified constant $\Delta$.

In $\S 2.1$ below, we state an assumption that clarifies how the first component of $V(\cdot)$ generates homophily and sparsity. In $\S 2.2$, we state a general restriction specifying how $S_{i j}$ may depend on $\boldsymbol{A}$. We also introduce an equilibrium selection mechanism since strategic interactions generally result in multiple equilibria (Sheng, 2020). Finally, in $\S 2.3$, we define the class of network moments for which provide a CLT.

\section{$2.1 \quad$ Homophily and Sparsity}

A common feature of social networks is homophily, the tendency for those with similar characteristics to associate. If the joint-surplus function $V(\cdot)$ is decreasing in the 


\section{Normal Approximation in Large Network Models}

first component, then it implies that nodes that are "socially dissimilar" in terms of positions $X_{i}$ are less likely to form links, which captures homophily in positions. Positions can abstractly represent node locations in an underlying "social space," as in latent-space models (Breza et al., 2020; Hoff et al., 2002). They may also represent attributes such as income and geographic location.

Another common feature is sparsity, meaning that the number of connections formed by the typical node is of smaller order than the number of nodes (Barabási, 2015; Chandrasekhar, 2016). This is often accomplished by scaling the sequence of models such that the expected degree of any node is asymptotically bounded:

$$
\frac{1}{n} \sum_{i=1}^{n} \sum_{j \neq i} \mathbf{E}\left[A_{i j}\right]=O(1)
$$

Our first assumption restricts the joint-surplus function $V(\cdot)$ and the distribution of $\zeta_{i j}$, so that the network generated by model (1) satisfies homophily and sparsity. Let $\bar{\Phi}_{\zeta}(z)=\mathbf{P}\left(\zeta_{i j} \geqslant z\right)$. Define $\bar{V}\left(r_{n}^{-1}\left\|X_{i}-X_{j}\right\|, \zeta_{i j}\right)=\sup _{s, z, z^{\prime}} V\left(r_{n}^{-1} \| X_{i}-\right.$ $\left.X_{j} \|, s, z, z^{\prime}, \zeta_{i j}\right)$, where the supremum is taken with respect to the support of $\left(S_{i j}, Z_{i}, Z_{j}\right)$ (which is identical across $i, j$ ). Finally, recall $d$ is the dimension of $X_{1}$.

Assumption 1 (Homophily and Sparsity). For any $\delta \in \mathbb{R}_{+}, \bar{V}(\delta, \cdot)$ is invertible in its second argument, and its inverse $\bar{V}^{-1}(\delta, \cdot)$ satisfies $\lim \sup _{\delta \rightarrow \infty} \delta^{-1} \log \bar{\Phi}_{\zeta}\left(\bar{V}^{-1}(\delta, 0)\right)<$ 0 . Furthermore, there exists $\kappa>0$ such that, for any $n \in \mathbb{N}$,

$$
r_{n} \equiv(\kappa / n)^{1 / d}
$$

Invertibility is satisfied, for example, if the random-utility shock $\zeta_{i j}$ is additively separable in $V(\cdot)$. Homophily is a consequence of the first sentence because $\mathbf{P}\left(A_{i j}=\right.$ $\left.1 \mid r_{n}^{-1}\left\|X_{i}-X_{j}\right\|=\delta\right) \leqslant \bar{\Phi}_{\zeta}\left(\bar{V}^{-1}(\delta, 0)\right)$, which is required to decrease exponentially with $\delta$. If $V(\cdot)$ is not too nonlinear in its first component and the distribution of $\zeta_{i j}$ has exponential tails, then this assumption is satisfied, as shown in the next example.

Example 3. Consider Example 1, and suppose $S_{i j}$ and $Z_{i}$ have uniformly bounded 


\section{LEUNG AND MOON}

support. Then for some constant $M$,

$$
\begin{aligned}
\mathbf{P}\left(V\left(r_{n}^{-1}\left\|X_{i}-X_{j}\right\|, S_{i j}, Z_{i}, Z_{j}, \zeta_{i j}\right)>0 \mid r_{n}^{-1}\left\|X_{i}-X_{j}\right\|=\delta\right) & \\
& \leqslant \mathbf{P}(\underbrace{M-\delta+\zeta_{i j}}_{\bar{V}\left(\delta, \zeta_{i j}\right)}>0)=\bar{\Phi}_{\zeta}(\underbrace{\delta-M}_{\bar{V}^{-1}(\delta, 0)}),
\end{aligned}
$$

which decays to zero exponentially with $\delta$ if the distribution of $\zeta_{i j}$ has exponential tails.

Sparsity holds if $r_{n}$ tends to zero at a certain rate with $n$. Intuitively, as $n$ increases, the number of opportunities to form links grows, which promotes density, whereas as $r_{n}$ decreases, the number of attractive partners decreases due to homophily, which promotes sparsity. Our choice (5) in Assumption 1 balances these two forces so that the expected degree remains asymptotically bounded but nonzero. Under this sequence, the expected degree is

$$
\begin{gathered}
\frac{1}{n} \sum_{i=1}^{n} \sum_{j \neq i} \mathbf{E}\left[A_{i j}\right]=(n-1) \mathbf{P}\left(V\left(r_{n}^{-1}\left\|X_{i}-X_{j}\right\|, S_{i j}, Z_{i}, Z_{j}, \zeta_{i j}\right)>0\right) \\
\quad \leqslant(n-1) \mathbf{P}\left(\zeta_{i j}>\bar{V}^{-1}\left(r_{n}^{-1}\left\|X_{i}-X_{j}\right\|, 0\right)\right) \\
=(n-1) r_{n}^{d} \int_{\mathbb{R}^{d}} \int_{\mathbb{R}^{d}} \mathbf{P}\left(\zeta_{i j}>\bar{V}^{-1}\left(\left\|x-x^{\prime}\right\|, 0\right)\right) f(x) f\left(x+r_{n}\left(x^{\prime}-x\right)\right) \mathrm{d} x \mathrm{~d} x^{\prime}
\end{gathered}
$$

by a change of variables. By (5), if $f$ is continuous, this converges to

$$
\kappa \int_{\mathbb{R}^{d}} \int_{\mathbb{R}^{d}} \bar{\Phi}_{\zeta}\left(\bar{V}^{-1}\left(\left\|x-x^{\prime}\right\|, 0\right)\right) f(x)^{2} \mathrm{~d} x^{\prime} \mathrm{d} x,
$$

which is finite because the integrand decays exponentially with $\left\|x-x^{\prime}\right\|$ by Assumption 1 .

\subsection{Strategic Interactions and Equilibrium Selection}

We next formally define the statistics $S_{i j}$ that capture strategic interactions. For any $H \subseteq \mathbb{N}$, let $r_{n}^{-1} \boldsymbol{T}_{H}=\left(\left(r_{n}^{-1} X_{i}, Z_{i}\right)\right)_{i \in H}$, the array of (scaled) types for nodes in $H$, and $\boldsymbol{\zeta}_{H}=\left(\zeta_{i j}: i \neq j, i, j \in H\right)$, the corresponding array of random-utility shocks. In the case where $H=\mathcal{N}_{n}$, we abbreviate $r_{n}^{-1} \boldsymbol{T}_{\mathcal{N}_{n}}=r_{n}^{-1} \boldsymbol{T}_{n}$ and $\boldsymbol{\zeta}_{\mathcal{N}_{n}}=\boldsymbol{\zeta}_{n}$. 


\section{Normal Approximation in Large Network Models}

For any $n \in \mathbb{N}$, we posit the existence of a vector-valued function $S_{n}(\cdot)$ such that

$$
S_{i j}=S_{n}\left(i, j, r_{n}^{-1} \boldsymbol{T}_{n}, \boldsymbol{A}\right)
$$

for any nodes $i, j$ and $S_{n}(\cdot)$ is invariant to permutations of node labels. ${ }^{2}$

Under (7), $S_{i j}$ is allowed to be any arbitrary function of the network and nodes' types. We next impose the common restriction that $S_{n}(\cdot)$ only depends on its arguments through the 1-neighborhoods of $i$ and $j$ in $\boldsymbol{A}$. For any $H \subseteq \mathcal{N}_{n}$, let $\boldsymbol{A}_{H}=\left(A_{k l}: k \neq l,\{k, l\} \subseteq H\right)$, the subnetwork of $\boldsymbol{A}$ on $H$. Recall from $\S 1$ that $\mathcal{N}_{\boldsymbol{A}}(i, 1)$ denotes $i$ 's 1-neighborhood.

Assumption 2 (Local Externalities). For any $n \in \mathbb{N}, i, j \in \mathcal{N}_{n}$ with $i \neq j$, and $H=\mathcal{N}_{\boldsymbol{A}}(i, 1) \cup \mathcal{N}_{\boldsymbol{A}}(j, 1)$,

$$
S_{n}\left(i, j, r_{n}^{-1} \boldsymbol{T}_{n}, \boldsymbol{A}\right)=S_{|H|}\left(i, j, r_{n}^{-1} \boldsymbol{T}_{H}, \boldsymbol{A}_{H}\right) .^{3}
$$

This states that $S_{i j}$ is only a function of nodes linked to either $i$ or $j$. Most statistics used in the literature satisfy this assumption, including those in Examples 1 and 2, which appear to be the most common choices (Christakis et al., 2020; Goldsmith-Pinkham and Imbens, 2013; Leung, 2019b; Mele, 2017; Sheng, 2020). Dependence of $S_{n}(\cdot)$ on types also allows for covariate-weighted versions of these examples, such as $\sum_{k=1}^{n} A_{i k} Z_{k}$.

The model thus far is incomplete since multiple networks $\boldsymbol{A}$ may satisfy (1) due to strategic interactions. Let $\mathcal{E}\left(r_{n}^{-1} \boldsymbol{T}_{n}, \boldsymbol{\zeta}_{n}\right)$ denote the set of such networks (the set of pairwise stable equilibria). To complete the model, we introduce a selection mechanism, which is the reduced-form mapping from primitives to the observed network.

Assumption 3 (Equilibrium Selection). For any $n \in \mathbb{N}$, an equilibrium exists in that $\mathcal{E}\left(r_{n}^{-1} \boldsymbol{T}_{n}, \boldsymbol{\zeta}_{n}\right)$ is non-empty. There exists a function $\lambda_{n}(\cdot)$ such that $\boldsymbol{A}=\lambda_{n}\left(r_{n}^{-1} \boldsymbol{T}_{n}, \boldsymbol{\zeta}_{n}\right)$, where $\lambda_{n}(\cdot)$ maps $\left(r_{n}^{-1} \boldsymbol{T}_{n}, \boldsymbol{\zeta}_{n}\right)$ to an element of $\mathcal{E}\left(r_{n}^{-1} \boldsymbol{T}_{n}, \boldsymbol{\zeta}_{n}\right)$.

The selection mechanism $\lambda_{n}(\cdot)$ denotes the latent social process by which nodes co-

\footnotetext{
${ }^{2}$ Formally, for any bijection $\pi: \mathcal{N}_{n} \rightarrow \mathcal{N}_{n}$, abuse notation by defining $\pi\left(r_{n}^{-1} \boldsymbol{T}_{n}\right)=$ $\left(\left(r_{n}^{-1} X_{\pi(i)}, Z_{\pi(i)}\right)\right)_{i \in \mathcal{N}_{n}}$ and similarly $\pi(\boldsymbol{A})$. Invariance of $S_{n}(\cdot)$ to permutations means $S_{n}\left(i, j, r_{n}^{-1} \boldsymbol{T}_{n}, \boldsymbol{A}\right)=S_{n}\left(\pi(i), \pi(j), \pi\left(r_{n}^{-1} \boldsymbol{T}_{n}\right), \pi(\boldsymbol{A})\right)$, which means that node labels are arbitrary.

${ }^{3}$ Strictly speaking, we require this to hold for any positive sequence $\left\{r_{n}\right\}_{n \in \mathbb{N}}$ for which $n r_{n}^{d} \rightarrow \kappa$, the constant defined in Assumption 1, but we will omit this for brevity in this and future assumptions.
} 


\section{LEUNG AND MOON}

ordinate on an equilibrium. The assumption is standard in the empirical games literature and also used by Sheng (2020). ${ }^{4}$

\subsection{Network Moments}

Our objective is to prove a CLT for network moments, which we define as averages of node statistics

$$
\frac{1}{n} \sum_{i=1}^{n} \psi_{n}\left(i, r_{n}^{-1} \boldsymbol{T}_{n}, \boldsymbol{\zeta}_{n}, \boldsymbol{A}\right),
$$

where $\psi_{n}(\cdot)$ is $\mathbb{R}^{d_{\psi}}$-valued.

Example 4 (Subnetwork Counts). A simple network moment is the average degree, for which $\psi_{i}\left(\mathcal{N}_{n}\right)$ is node $i$ 's degree $\sum_{j=1}^{n} A_{i j}$. This is proportional to the dyad count (number of links), and more generally, we can count any other connected subnetwork, such as the number of triangles, $k$-stars, or complete networks on $k$-tuples. For instance, the triangle count is proportional to $\sum_{i \neq j \neq k} A_{i j} A_{j k} A_{i k}$, with corresponding node statistic $\sum_{j, k: i \neq j \neq k} A_{i j} A_{j k} A_{i k}$. See $\S \mathrm{B}$ for a formal definition of subnetwork counts.

Subnetwork counts are useful for structural inference. Suppose $V(\cdot)$ is known up to some vector of parameters $\theta_{0}$. Sheng (2020) defines an identified set for $\theta_{0}$ in terms of moment inequalities of the form

$$
\frac{1}{n} \sum_{i=1}^{n} \mathbf{E}\left[G_{i}-H_{i}\left(\theta_{0}\right)\right] \leqslant \mathbf{0},
$$

where $\sum_{i=1}^{n} G_{i}$ is a vector of subnetwork counts and $H_{i}(\cdot)$ is a deterministic function of the observed subvector of $\left(\left(X_{i}, Z_{i}\right)\right)_{i=1}^{k}$, where $k$ is the size of the subnetwork being counted (e.g. 3 for triangles). Under the assumptions of Sheng $(2020), H_{i}(\cdot)$ can be computed by simulation.

\footnotetext{
${ }^{4}$ In the empirical games literature, it is perhaps more common to represent the equilibrium selection mechanism as a distribution on $\mathcal{E}\left(r_{n}^{-1} \boldsymbol{T}_{n}, \boldsymbol{\zeta}_{n}\right)$ conditional on the primitives $\left(r_{n}^{-1} \boldsymbol{T}_{n}, \boldsymbol{\zeta}_{n}\right)$. Our representation is equivalent. First note that, if we were to extend the domain of $\lambda_{n}(\cdot)$ to incorporate an additional random vector $\nu$ independent of the primitives, then any desired conditional distribution can be represented as $\mathbf{P}\left(\lambda_{n}\left(r_{n}^{-1} \boldsymbol{T}_{n}, \boldsymbol{\zeta}_{n}, \nu\right)=\boldsymbol{A} \mid r_{n}^{-1} \boldsymbol{T}_{n}, \boldsymbol{\zeta}_{n}\right)$. Second, it is without loss of generality to not explicitly include $\nu$ in the domain because we can always include in each $Z_{i}$ random variables that are payoff-irrelevant in the sense that they do not enter $V(\cdot)$ and $S_{n}(\cdot)$.
} 


\section{Normal Approximation in Large Network Models}

More generally, we consider the following class of node statistics that includes the previous example. Recall that $\mathcal{N}_{\boldsymbol{A}}(i, K)$ is $i$ 's $K$-neighborhood.

Assumption 4 (K-Locality). For some integer $K$ and any $n \in \mathbb{N}, i \in \mathcal{N}_{n}$, and $H=\mathcal{N}_{\boldsymbol{A}}(i, K)$,

$$
\psi_{n}\left(i, r_{n}^{-1} \boldsymbol{T}_{n}, \boldsymbol{\zeta}_{n}, \boldsymbol{A}\right)=\psi_{|H|}\left(i, r_{n}^{-1} \boldsymbol{T}_{H}, \boldsymbol{\zeta}_{H}, \boldsymbol{A}_{H}\right) .
$$

This states that $\psi_{i}\left(\mathcal{N}_{n}\right)$ only depends on the types, random-utility shocks, and the subnetwork of nodes in $i$ 's $K$-neighborhood. The average degree and triangle count both satisfy this for $K=1$, and more generally, subnetwork counts satisfy this for appropriate choices of $K$. A more complex example is the number of nodes at most path distance $D$ from node $i$, which satisfies this assumption for $K=D$.

Example 5. Returning to Example $4, n^{-1} \sum_{i=1}^{n} H_{i}\left(\theta_{0}\right)$ is proportional to a U-statistic of order $k$, so by the Hoeffding decomposition, $n^{-1} \sum_{i=1}^{n} H_{i}\left(\theta_{0}\right)=n^{-1} \sum_{i=1}^{n} \tilde{H}_{i}(\theta)+$ $o_{p}\left(n^{-1 / 2}\right)$ for some $\tilde{H}_{i}\left(\theta_{0}\right)$ that is a non-random function of $i$ 's type $\left(X_{i}, Z_{i}\right)$ (e.g. Leung, 2015a, Proposition B.2). Hence, $\tilde{H}_{i}\left(\theta_{0}\right)$ satisfies Assumption 4 for $K=0$. Since $G_{i}$ is also a node statistic satisfying Assumption 4 , so is $G_{i}-\tilde{H}_{i}(\theta)$, so our results apply to the sample moments $n^{-1} \sum_{i=1}^{n}\left(G_{i}-H_{i}\left(\theta_{0}\right)\right)$.

Finally, without loss of generality, we assume that, for any $i \in \mathcal{N}_{n}$ and $x \in \mathbb{R}^{d}$,

$$
\begin{aligned}
& \psi_{n}\left(i, r_{n}^{-1} \boldsymbol{T}_{n}, \boldsymbol{\zeta}_{n}, \boldsymbol{A}\right)=\psi_{n}\left(i,\left(\left(r_{n}^{-1} X_{j}+x, Z_{j}\right)\right)_{j=1}^{n}, \boldsymbol{\zeta}_{n}, \boldsymbol{A}\right) \quad \text { and } \\
& \left.\lambda_{n}\left(r_{n}^{-1} \boldsymbol{T}_{n}, \boldsymbol{\zeta}_{n}\right)=\lambda_{n}\left(\left(r_{n}^{-1} X_{j}+x, Z_{j}\right)\right)_{j=1}^{n}, \boldsymbol{\zeta}_{n}\right) .
\end{aligned}
$$

This says that scaled positions $\left\{r_{n}^{-1} X_{i}\right\}_{i=1}^{n}$ only enter node statistics and the selection mechanism through distances $r_{n}^{-1}\left\|X_{i}-X_{j}\right\|$, as distances are invariant to shifting positions by $x$. Because $X_{i}$ and $Z_{i}$ may be arbitrarily dependent, $X_{i}$ may also enter the model as a subvector of $Z_{i}$, so this is without loss of generality.

\section{Stabilization}

This section establishes a CLT under a high-level weak-dependence condition ("stabilization") and discusses the proof technique. In $\S 4$, we provide primitive sufficient conditions for stabilization, so those interested in the application of the result to 


\section{LEUNG AND MOON}

strategic network formation may wish to skip to that section, most of which can be read independently.

Recall from Assumption 3 that, for any $H \subseteq \mathcal{N}_{n}, \lambda_{|H|}\left(r_{n}^{-1} \boldsymbol{T}_{H}, \boldsymbol{\zeta}_{H}\right)$ is the selected equilibrium network on $H$ under the counterfactual in which the set of nodes is $H$, rather than $\mathcal{N}_{n}$. Abusing notation, we define $i$ 's counterfactual node statistic

$$
\psi_{i}(H) \equiv \psi_{|H|}\left(i, r_{n}^{-1} \boldsymbol{T}_{H}, \boldsymbol{\zeta}_{H}, \lambda_{|H|}\left(r_{n}^{-1} \boldsymbol{T}_{H}, \boldsymbol{\zeta}_{H}\right)\right) .
$$

The counterfactual interpretation is due entirely to the last component. It is useful to contrast this with

$$
\psi_{|H|}\left(i, r_{n}^{-1} \boldsymbol{T}_{H}, \boldsymbol{\zeta}_{H}, \boldsymbol{A}_{H}\right)
$$

which is not counterfactual and can be computed directly from the realized primitives by restricting to the observed subnetwork on $H$. For example, if $\psi_{i}\left(\mathcal{N}_{n}\right)$ is the degree of node $i$ on the full network, then (10) is $i$ 's degree on the subnetwork restricted to $H$. Generally, (10) differs from (9) because the networks $\boldsymbol{A}_{H}$ and $\lambda_{|H|}\left(r_{n}^{-1} \boldsymbol{T}_{H}, \boldsymbol{\zeta}_{H}\right)$ do not coincide. This is due to strategic interactions, which induce nodes in $H$ to form different links when nodes $\mathcal{N}_{n} \backslash H$ are absent from the model.

For any $B \subseteq \mathbb{R}^{d}$ (recalling that $d$ is the dimension of $X_{1}$ ), let $\mathcal{N}_{n}(B)=\{i \in$ $\left.\mathcal{N}_{n}: X_{i} \in B\right\}$, the set of nodes positioned in $B$.

Definition 1. For any $n \in \mathbb{N}$ and $i \in \mathcal{N}_{n}$, the radius of stabilization

$$
\boldsymbol{R}_{i}\left(n, r_{n}\right) \equiv \boldsymbol{R}\left(i, r_{n}^{-1} \boldsymbol{T}_{n}, \boldsymbol{\zeta}_{n}, \lambda_{n}\right)
$$

is the smallest random variable $R \geqslant 0$ such that $\psi_{i}\left(\mathcal{N}_{n}\right)=\psi_{i}\left(\mathcal{N}_{n}\left(\mathcal{B}_{i}\right)\right)$ for all sets $\mathcal{B}_{i} \subseteq \mathbb{R}^{d}$ containing $B\left(r_{n}^{-1} X_{i}, R\right)$.

The radius of stabilization is essentially the smallest radius $R$ such that $i$ 's node statistic has the same value under all counterfactuals that remove from the model nodes positioned outside of a neighborhood $B\left(r_{n}^{-1} X_{i}, R\right)$ of $i$ 's position. The model primitives (types and random-utility shocks) are fixed under these counterfactuals.

The main idea is as follows. If this radius were "small," then $\psi_{i}\left(\mathcal{N}_{n}\right)$ would primarily depend on a small set of nodes positioned near $i$, in which case node statistics would be weakly dependent. To establish a law of large numbers (LLN), Leung (2019b) requires the radius to be $O_{p}(1)$, analogous to a condition due to Penrose and Yukich 


\section{Normal Approximation in Large Network Models}

(2003). However, as subsequent work by Penrose and coauthors established, a CLT requires stronger control on the distribution of the radius, in particular the thickness of its tails. We next adapt their ideas to our setting, which accommodates models with strategic interactions such as (1).

\subsection{Abstract CLT}

For technical reasons, we need to consider sequences of models indexed by $n$ in which the network size is not actually $n$ but rather of the same asymptotic order as $n$. Let $\left\{N_{n}\right\}_{n \in \mathbb{N}}$ be a sequence of random variables independent of all model primitives such that

$$
N_{n} \sim \operatorname{Poisson}(n)
$$

A de-Poissonization argument discussed in $\S 3$ requires us to consider two different network sizes: a random size $N_{n}+k$, where $k$ is a constant, and a non-random size $m+k$, where $m=m_{n}$ with $m_{n} / n \rightarrow c \in(0, \infty)$. Our original setup with $n$ nodes corresponds to $m=n$ and $k=0$, and no intuition is lost to the reader who only considers this case. The need to consider models with different network sizes will be clarified in the proof sketch in $\S 3.2$.

Let $f$ be the density of $X_{1}, \operatorname{supp}(f)$ be the support of $f, \operatorname{supp}(f)^{k}=\times_{i=1}^{k} \operatorname{supp}(f)$, and $\boldsymbol{X}_{k}=\left(X_{j}\right)_{j=1}^{k}$.

Assumption 5 (Exponential Stabilization). For any $k \in\{1, \ldots, 4\}$ and $k^{\prime} \in\{1,2\}$, there exist $n_{0}, \epsilon>0$ such that $\lim \sup _{w \rightarrow \infty} w^{-1} \max \left\{\log \tau_{b, \epsilon}(w), \log \tau_{p}(w)\right\}<0$, where

$$
\begin{aligned}
& \tau_{b, \epsilon}(w)=\sup _{n>n_{0}} \sup _{m \in((1-\epsilon) n,(1+\epsilon) n)} \sup _{\boldsymbol{x}_{k} \in \operatorname{supp}(f)^{k}} \mathbf{P}\left(\boldsymbol{R}_{1}\left(m+k, r_{n}\right)>w \mid \boldsymbol{X}_{k}=\boldsymbol{x}_{k}\right), \\
& \tau_{p}(w)=\sup _{n>n_{0}} \sup _{\boldsymbol{x}_{k^{\prime}} \in \operatorname{supp}(f)^{k^{\prime}}} \mathbf{P}\left(\boldsymbol{R}_{1}\left(N_{n}+k^{\prime}, r_{n}\right)>w \mid \boldsymbol{X}_{k^{\prime}}=\boldsymbol{x}_{k^{\prime}}\right) .
\end{aligned}
$$

The assumption says that the distribution of the radius of stabilization has an exponential tail under various models with $N_{n}+k$ and $m+k$ nodes. The use of node 1 in, for example, $\boldsymbol{R}_{1}\left(m+k, r_{n}\right)$ is arbitrary since nodes are exchangeable. The condition is analogous to those used by Penrose and Yukich (2005) and Penrose (2007), and our method of proof for the result below is based on theirs but with important differences in setup discussed in $\S 3.3$. 


\section{LEUNG AND MOON}

Contrast Assumption 5 with the more familiar concept of $M$-dependence, which states that an observation is only correlated with nodes in some non-random neighborhood of known radius $M$. Stabilization generalizes this to allow neighborhoods to be node-specific, random, and potentially highly complex functions of the primitives, including unobservables, as will be clear from the next subsection. In general, bounding the size of this set is far from trivial, and one of our main contributions (discussed in $\S 4.4)$ is to demonstrate that branching processes can be used for this purpose.

The next assumption is a moment condition. Let $\|x\|_{\infty}$ denote the component-wise maximum of a vector $x$.

Assumption 6 (Bounded Moments). There exist $p>2, \epsilon>0, M<\infty$, and $n_{0} \in \mathbb{N}$ such that for all $n>n_{0}, k \in\{1,2\}, m \in((1-\epsilon) n,(1+\epsilon) n)$, and $\boldsymbol{x}_{k} \in \operatorname{supp}(f)^{k}$,

$\max \left\{\mathbf{E}\left[\left\|\psi_{1}\left(\mathcal{N}_{m+k}\right)\right\|_{\infty}^{p} \mid \boldsymbol{X}_{k}=\boldsymbol{x}_{k}\right], \mathbf{E}\left[\left\|\psi_{1}\left(\mathcal{N}_{N_{n}+k} \cap\left(H_{n} \cup\{1\}\right)\right)\right\|_{\infty}^{p} \mid \boldsymbol{X}_{k}=\boldsymbol{x}_{k}\right]\right\}<M$

for any sequence of random sets $H_{n} \subseteq\left\{2, \ldots, N_{n}+1\right\}$ that may depend on $\left(X_{i}\right)_{i=1}^{N_{n}+1}$. Additionally, $\operatorname{supp}(f)$ is a bounded set.

This says that node statistics have uniformly bounded $p>2$ moments. Proposition B.1 verifies this for subnetwork counts (Example 4).

Let $\boldsymbol{\Sigma}_{n}=n^{-1} \operatorname{Var}\left(\sum_{i=1}^{n} \psi_{i}\left(\mathcal{N}_{n}\right)\right), \lambda_{\min }\left(\boldsymbol{\Sigma}_{n}\right)$ its smallest eigenvalue, $\left\|\boldsymbol{\Sigma}_{n}\right\|$ its entrywise maximum, and $\boldsymbol{I}_{n}$ the identity matrix with the same dimensions as $\boldsymbol{\Sigma}_{n}$.

Theorem 1. Under Assumptions 5 and $6, \sup _{n}\left\|\boldsymbol{\Sigma}_{n}\right\|<\infty$, and if additionally $\liminf _{n \rightarrow \infty} \lambda_{\min }\left(\Sigma_{n}\right)>0$, then

$$
\boldsymbol{\Sigma}_{n}^{-1 / 2} \frac{1}{\sqrt{n}} \sum_{i=1}^{n}\left(\psi_{i}\left(\mathcal{N}_{n}\right)-\mathbf{E}\left[\psi_{i}\left(\mathcal{N}_{n}\right)\right]\right) \stackrel{d}{\longrightarrow} \mathcal{N}\left(\mathbf{0}, \boldsymbol{I}_{n}\right)
$$

ProOF. See §SA.1.

Other than the independence assumptions at the top of $\S 2$ and the normalization (8), the proof does not utilize the assumptions in $\S 2$. While Assumption 3 seems to be used implicitly in the definition of $\psi_{i}\left(\mathcal{N}_{n}\right)$, the proof of Theorem 1 only makes use of the fact that $\lambda_{n}(\cdot)$ is a deterministic function of the structural primitives, not even that its range is the space of networks on $n$ nodes. As such, the result can be 


\section{Normal Approximation in Large Network Models}

applied more broadly to other network models. For example, in Leung (2019a), $\lambda_{n}(\cdot)$ is the reduced-form mapping that takes as input the primitives and outputs both the network and outcome of a social interactions model given the network.

The difficulty with applying Theorem 1 is verifying Assumption 5. In $\S 4$, we introduce primitive conditions to verify the assumption for model (1).

\subsection{Outline of Proof}

Step 1. We first establish a CLT for the "Poissonized" model in which the number of nodes is $N_{n}$ defined in (11), so-called because $\left\{X_{i}\right\}_{i=1}^{N_{n}}$ has the same distribution as a Poisson point process with intensity function $n f(\cdot)$ (Penrose, 2003, Proposition 1.5). Specifically, we show

$$
\tilde{\boldsymbol{\Sigma}}_{n}^{-1 / 2} \frac{1}{\sqrt{n}}\left(\sum_{i=1}^{N_{n}} \psi_{i}\left(\mathcal{N}_{N_{n}}\right)-\mathbf{E}\left[\sum_{i=1}^{N_{n}} \psi_{i}\left(\mathcal{N}_{N_{n}}\right)\right]\right) \stackrel{d}{\longrightarrow} \mathcal{N}\left(\mathbf{0}, \boldsymbol{I}_{n}\right),
$$

where $\tilde{\boldsymbol{\Sigma}}_{n}=n^{-1} \operatorname{Var}\left(\sum_{i=1}^{N_{n}} \psi_{i}\left(\mathcal{N}_{N_{n}}\right)\right)$. This is simpler to prove directly because, unlike $\left\{X_{i}\right\}_{i=1}^{n}$, the Poisson process $\left\{X_{i}\right\}_{i=1}^{N_{n}}$ possesses a well-known spatial independence property: for any disjoint subsets $S_{1}, S_{2} \subseteq \mathbb{R}^{d},\left|\left\{X_{i}\right\}_{i=1}^{N_{n}} \cap S_{1}\right| \Perp\left|\left\{X_{i}\right\}_{i=1}^{N_{n}} \cap S_{2}\right|$.

We prove (13) by adapting a theorem due to Penrose and Yukich (2005) (see our Theorem SA.1.1). We proceed by first partitioning the support of $X_{1}$ into cubes $Q_{1}, \ldots, Q_{V_{n}}$ of slowly growing volume and representing the moment $n^{-1} \sum_{i=1}^{n} \psi_{i}\left(\mathcal{N}_{N_{n}}\right)$

as a double sum over cubes and nodes within cubes $n^{-1} \sum_{i=1}^{V_{n}} \sum_{j=1}^{N_{i}} \psi_{i j}\left(\mathcal{N}_{N_{n}}\right)$, where $N_{i}$ is the number of nodes positioned in $Q_{i}$ and $i j$ indexes the $j$ th node positioned in cube $i$. Spatial independence of the Poisson process implies independence of node positions across cubes. On the other hand, node statistics are complex functionals of the process and hence are generally not independent across cubes.

However, since stabilization implies that node statistics $\psi_{i j}\left(\mathcal{N}_{n}\right)$ are primarily determined by nodes relatively proximate to $i j$, and hence, cubes relatively proximate $Q_{i}$, it can be shown that the dependence structure of $\left\{\sum_{j=1}^{N_{i}} \psi_{i j}\left(\mathcal{N}_{N_{n}}\right)\right\}_{i=1}^{V_{n}}$ is "approximately" characterized by a certain "dependency graph." This is a network in which, roughly speaking, two observations are linked if and only if they are dependent. In our case, observations are cubes, which we connect if and only if they are relatively proximate. Careful construction of the cubes ensures a small approximation error for the dependency graph characterization, and applying a CLT for dependency graphs 


\section{LEUNG AND MOON}

(Chen and Shao, 2004) delivers the result. For precise details, see §SA.1.1.

Step 2. Since $N_{n} / n \stackrel{p}{\longrightarrow} 1,\left\{X_{i}\right\}_{i=1}^{n}$ and $\left\{X_{i}\right\}_{i=1}^{N_{n}}$ should be "similar," so given (13), we expect a similar result for the original model. The second "de-Poissonization" step of the proof, which follows Penrose (2007), shows that this intuition is correct, provided we properly adjust $\tilde{\boldsymbol{\Sigma}}_{n}$ downward to obtain the correct variance $\boldsymbol{\Sigma}_{n}$ (see our Theorem SA.1.2). Intuitively, an adjustment is required because $N_{n}$ contributes extra randomness to the asymptotic distribution.

More specifically, define the add-one cost

$$
\Xi_{n}=\psi_{n+1}\left(\mathcal{N}_{n+1}\right)+\sum_{i=1}^{n}\left(\psi_{i}\left(\mathcal{N}_{n+1}\right)-\psi_{i}\left(\mathcal{N}_{n}\right)\right)
$$

This is the aggregate counterfactual impact on the total $\sum_{i=1}^{n} \psi_{i}\left(\mathcal{N}_{n}\right)$ from adding a new node labeled $n+1$ to the model. The first term is the direct effect of adding $n+1$, which is its own node statistic. The second term is the indirect effect, which is the new node's impact on the statistics of all other nodes. A key step of the proof establishes that

$$
\begin{aligned}
n^{-1 / 2}\left(\sum_{i=1}^{N_{n}} \psi_{i}\left(\mathcal{N}_{N_{n}}\right)-\mathbf{E}\left[\sum_{i=1}^{N_{n}} \psi_{i}\left(\mathcal{N}_{N_{n}}\right)\right]\right) & n^{-1 / 2}\left(\sum_{i=1}^{n} \psi_{i}\left(\mathcal{N}_{n}\right)-\mathbf{E}\left[\sum_{i=1}^{n} \psi_{i}\left(\mathcal{N}_{n}\right)\right]\right) \\
& +n^{-1 / 2}\left(N_{n}-n\right) \mathbf{E}\left[\Xi_{N_{n}}\right]+o_{p}(1) .
\end{aligned}
$$

This may be viewed as a first-order expansion with respect to the number of nodes, comparing $N_{n}$ to $n$. The "derivative" is $\mathbf{E}\left[\Xi_{N_{n}}\right]$ since it captures the change in moments as a result of a unit increment in the number of nodes. By (13), the left-hand side is asymptotically normal, and by the Poisson CLT, so is $n^{-1 / 2}\left(N_{n}-n\right) \mathbf{E}\left[\Xi_{N_{n}}\right]$. Because $N_{n}$ is independent of all other primitives, we can then establish that the first term on the right-hand side is asymptotically normal by an argument using characteristic functions; for details see the end of $\S \mathrm{SA} .1 .2$. 


\section{Normal Approximation in Large Network Models}

\subsection{Related Literature}

The proof is closely based on arguments in Penrose and Yukich (2005) and Penrose (2007), whose results pertain to geometric graphs without strategic interactions. The innovation in Theorem 1 is primarily conceptual, namely, the recognition that an appropriate modification of stabilization allows us to adapt their results to econometric models of interest. Our main technical innovation is the use of branching processes in the proof of Theorem 2 in $\S 4$ to derive primitive conditions for stabilization in models with strategic interactions. Leung (2019b) uses branching processes to establish an LLN, while we tackle the more difficult task of proving a CLT, which requires us to establish new exponential tail bounds for the radius of stabilization (Lemma A.4).

The setup and assumptions used in Penrose's work are not directly applicable to our setting, so we cannot simply verify their conditions. However, we show that their proofs can be translated to our setting, which differs in three main aspects. The first is the definition of the radius of stabilization. We reformulate the definition in terms of counterfactual models (9) and require invariance of $i$ 's node statistic to the removal of nodes outside of $\mathcal{N}_{n}\left(\mathcal{B}_{i}\right)$. In contrast, the literature's definitions demand invariance to the removal and addition of new nodes. Invariance to addition is typically violated in the models we study due to strategic interactions. Second, $X_{i}$ may be correlated with $Z_{i}$ in our setup, whereas the literature requires independence, but this turns out to have little effect on the proofs. Third, our model includes pair-specific shocks $\zeta_{i j}$, which intuitively poses little problem due to the high degree of independence. They are independent across pairs, whereas type pairs $\left(\left(X_{i}, Z_{i}\right),\left(X_{j}, Z_{j}\right)\right)$ are correlated across pairs sharing a common node, for example $(i, j)$ and $(i, k)$.

\section{CLT for Network Formation}

Theorem 1 establishes a CLT in an abstract setup under an exponential stabilization condition, which is a high-level formulation of weak dependence for the node statistics $\left\{\psi_{i}\left(\mathcal{N}_{n}\right)\right\}_{i=1}^{n}$. This section provides a CLT for the network formation model in $\S 2$ under primitive conditions. We also outline a methodology for deriving primitive conditions for stabilization in other network models. 


\section{LEUNG AND MOON}

\subsection{Strength of Interactions}

Consider the following measure of the strength of strategic interactions:

$$
\begin{aligned}
p_{r_{n}}\left(X_{i}, Z_{i}, X_{j}, Z_{j}\right) & =\mathbf{P}\left(\sup _{s} V\left(r_{n}^{-1}\left\|X_{i}-X_{j}\right\|, s, Z_{i}, Z_{j}, \zeta_{i j}\right)>0 \mid X_{i}, Z_{i}, X_{j}, Z_{j}\right) \\
& -\mathbf{P}\left(\inf _{s} V\left(r_{n}^{-1}\left\|X_{i}-X_{j}\right\|, s, Z_{i}, Z_{j}, \zeta_{i j}\right)>0 \mid X_{i}, Z_{i}, X_{j}, Z_{j}\right)
\end{aligned}
$$

(assuming measurability). This is the effect on link formation of changing $S_{i j}$ from its "lowest" to its "highest" possible value, conditional on types. In other words, it is the maximal change in linking probability induced by the strategic interactions component of $V(\cdot)$.

Let $d_{z}$ be the dimension of $Z_{i}$ and $\Phi_{z}(\cdot \mid x)$ the conditional distribution of $Z_{i}$ given $X_{i}=x$. Recall that $f$ is the density of $X_{1}$. For any $h: \mathbb{R}^{d} \times \mathbb{R}^{d_{z}} \rightarrow \mathbb{R}$, define the mixed norm

$$
\|h\|_{\mathbf{m}}=\sup _{x \in \mathbb{R}^{d}}\left(\int_{\mathbb{R}^{d z}} h(x, z)^{2} \mathrm{~d} \Phi^{*}(z)\right)^{1 / 2},
$$

where $\Phi^{*}(\cdot)$ is a distribution on $\mathbb{R}^{d_{z}}$ given in the next assumption.

Assumption 7 (Subcriticality). There exists a distribution $\Phi^{*}(\cdot)$ on $\mathbb{R}^{d_{z}}$ such that, for any $(x, z) \in \operatorname{supp}\left(X_{1}, Z_{1}\right)$ and $x^{\prime} \in \operatorname{supp}(f)$,

$$
\int_{\mathbb{R}^{d z}} p_{1}\left(x, z ; x^{\prime}, z^{\prime}\right) d \Phi_{z}\left(z^{\prime} \mid x^{\prime}\right) \leqslant \int_{\mathbb{R}^{d z}} p_{1}\left(x, z ; x^{\prime}, z^{\prime}\right) d \Phi^{*}\left(z^{\prime}\right)
$$

Furthermore, for $\bar{f}=\sup _{x \in \mathbb{R}^{d}} f(x)$ and $\kappa$ in $(5)$,

$$
\left\|h_{\boldsymbol{D}}\right\|_{\mathbf{m}}<1 \quad \text { where } h_{\boldsymbol{D}}(x, z)=\kappa \bar{f} \int_{\mathbb{R}^{d}}\left(\int_{\mathbb{R}^{d z}} p_{1}\left(x, z ; x^{\prime}, z^{\prime}\right)^{2} d \Phi^{*}\left(z^{\prime}\right)\right)^{1 / 2} d x^{\prime}
$$

The first equation is a regularity condition. The substantive requirement is $\left\|h_{\boldsymbol{D}}\right\|_{\mathbf{m}}<$ 1 , which is a slightly stronger version of Assumption 6 of Leung (2019b). This restricts the partial equilibrium effect of strategic interactions because (15) captures the direct effect of $S_{i j}$ on $A_{i j}$ but not subsequent adjustments in the network that lead to a new equilibrium. Simulation results in Leung (2019b) show that the general equilibrium effect of strategic interactions can still be substantive under this assumption.

Requiring $\left\|h_{\boldsymbol{D}}\right\|_{\mathbf{m}}<1$ is the network analog of well-known weak-dependence con- 


\section{Normal Approximation in Large Network Models}

ditions for linear spatial or temporal autoregressive models. These require the magnitude of the autoregressive parameter to be bounded below one, which restricts the degree of spatial or temporal dependence. In the linear-in-means model, this corresponds to the requirement $|\beta|<1$, where $\beta$ is the endogenous peer effect (Bramoullé et al., 2009). For nonlinear models, deriving an analogous condition is necessarily more complicated; see for instance equation (9) of de Jong and Woutersen (2011), which pertains to dynamic binary choice models. Their condition has some similarities to ours, but ours is significantly more complicated to derive since we study a simultaneousequations model with multiple equilibria.

Sheng (2020) does not impose Assumption 7 because she considers the case of many small independent networks. Our condition is as primitive as possible at this level of generality, but we may obtain further insight by specializing to a linear model with normal errors.

Example 6. Consider the following variant of Example 3:

$$
V\left(r_{n}^{-1}\left\|X_{i}-X_{j}\right\|, S_{i j}, Z_{i}, Z_{j}, \zeta_{i j}\right)=\theta_{1}+S_{i j}^{\prime} \theta_{2}-\theta_{3}\left(r_{n}^{-1}\left\|X_{i}-X_{j}\right\|\right)^{2}+\zeta_{i j},
$$

where $X_{i} \sim \mathcal{U}\left([0,1]^{2}\right), \zeta_{i j} \sim \mathcal{N}(0,1)$, and $S_{i j}$ has uniformly bounded support, so that $\left|S_{i j}^{\prime} \theta_{2}\right| \leqslant \bar{S}^{\prime} \theta_{2}$ for some vector of constants $\bar{S}$. Then

$$
\begin{aligned}
h_{\boldsymbol{D}}\left(x^{\prime}, z^{\prime}\right) & =\kappa \bar{f} \int_{\mathbb{R}^{2}} \mathbf{P}\left(-\bar{S}^{\prime} \theta_{2}<\theta_{1}-\theta_{3}\left\|x-x^{\prime}\right\|^{2}+\zeta_{12} \leqslant 0\right) \mathrm{d} x \\
& =\kappa \int_{\mathbb{R}^{2}}\left[\Phi\left(\theta_{1}+\bar{S}^{\prime} \theta_{2}-\theta_{3}\|x\|^{2}\right)-\Phi\left(\theta_{1}-\theta_{3}\|x\|^{2}\right)\right] \mathrm{d} x \\
& =2 \kappa \pi \int_{0}^{\infty} z\left[\Phi\left(\theta_{1}+\bar{S}^{\prime} \theta_{2}-\theta_{3} z^{2}\right)-\Phi\left(\theta_{1}-\theta_{3} z^{2}\right)\right] \mathrm{d} z
\end{aligned}
$$

by a change of variables to polar coordinates, where $\pi$ is the transcendental constant. Let $h(x)=x \Phi(x)+\phi(x)$, where $\Phi(\cdot)$ and $\phi(\cdot)$ are the normal CDF and PDF, respectively. The integral has a closed-form expression, resulting in

$$
\left\|h_{\boldsymbol{D}}\right\|_{\boldsymbol{m}}=\frac{\kappa \pi}{\theta_{3}}\left(h\left(\theta_{1}+\bar{S}^{\prime} \theta_{2}\right)-h\left(\theta_{1}\right)\right) .
$$

In order for the right-hand side to be less than one, which is sufficient for Assumption 7, $\theta_{2}$ must not be too large in magnitude, which is a direct restriction on the strength of 


\section{LEUNG AND MOON}

strategic interactions. Similarly, in the case of Sheng's payoff function (3), Assumption 7 implicitly restricts the magnitudes of $\gamma_{1}$ and $\gamma_{2}$, which is analogous to standard restrictions discussed above on autoregressive parameters or the endogenous peer effect.

\subsection{Strategic Neighborhoods}

We use branching process theory to formulate Assumption 7. Leung (2019b) derives a similar condition, which ensures that a certain branching process has asymptotically bounded size (the "subcritical" regime), in which case node statistics are weakly dependent and an LLN may be derived. Assumption 7 slightly strengthens this condition, and the technical details are more involved because we seek to establish a CLT. For this, we need not just asymptotic boundedness but also appropriate tail bounds on the size of the branching process.

We next introduce some definitions to explain the intuition. They are also needed to state our second weak-dependence condition below. Let

$$
\begin{aligned}
D_{i j}=1\left\{\left\{\sup _{s} V\left(r_{n}^{-1}\left\|X_{i}-X_{j}\right\|, s, Z_{i}, Z_{j}, \zeta_{i j}\right)>0\right\}\right. & \\
& \left.\cap\left\{\inf _{s} V\left(r_{n}^{-1}\left\|X_{i}-X_{j}\right\|, s, Z_{i}, Z_{j}, \zeta_{i j}\right) \leqslant 0\right\}\right\},
\end{aligned}
$$

whose conditional expectation is (15). If $\inf _{s} V\left(r_{n}^{-1}\left\|X_{i}-X_{j}\right\|, s, Z_{i}, Z_{j}, \zeta_{i j}\right)>0$, then the link between $i$ and $j$ is robust in the sense that the pairwise stability condition (1) holds regardless of the state of the network $\boldsymbol{A}$, which enters $V(\cdot)$ only through $S_{i j}$. Likewise, if $\sup _{s} V\left(r_{n}^{-1}\left\|X_{i}-X_{j}\right\|, s, Z_{i}, Z_{j}, \zeta_{i j}\right) \leqslant 0$, the potential link is robustly absent in the sense that, regardless of the network state, no link forms. In either case, $D_{i j}=0$. If instead $D_{i j}=1$, then $A_{i j}$ is non-robust in the sense that changes to the ambient network may well affect the pairwise stability of $A_{i j}$.

Let $\boldsymbol{D}$ be the $n \times n$ matrix with $i j$ th entry $D_{i j}$ for $i \neq j$ and $D_{i i}=0$ for all $i$. Viewing $\boldsymbol{D}$ as a network, let $C_{i}$ denote $i$ 's component in $\boldsymbol{D}$, recalling from $\S 1$ that a component is a connected subnetwork that is disconnected from the rest of the network. Define i's strategic neighborhood

$$
C_{i}^{+}=C_{i} \cup\left\{j \in \mathcal{N}_{n}: \max _{k \in C_{i}} \inf _{s} V\left(r_{n}^{-1}\left\|X_{j}-X_{k}\right\|, s, Z_{j}, Z_{k}, \zeta_{j k}\right)>0\right\},
$$

which adds to $C_{i}$ the set of all nodes that possess a robust link to some member 


\section{Normal Approximation in Large Network Models}

of $C_{i}$. Consider, for example, the network depicted in Figure 1, where dotted lines represent non-robust links (those of the network $\boldsymbol{D}$ ), solid lines represent robust links, and the absence of a line between two nodes represents a robustly absent link. The observed network $\boldsymbol{A}$ is a subnetwork of the depicted network since $A_{i j}$ may be 1 or 0 if $D_{i j}=1$. The components of $\boldsymbol{D}$ are the two islands obtained by removing the solid line: $\{1,2,3\}$ and $\{4,5,6\}$. The strategic neighborhoods are $\{1,2,3,4\}$ and $\{3,4,5,6\}$.

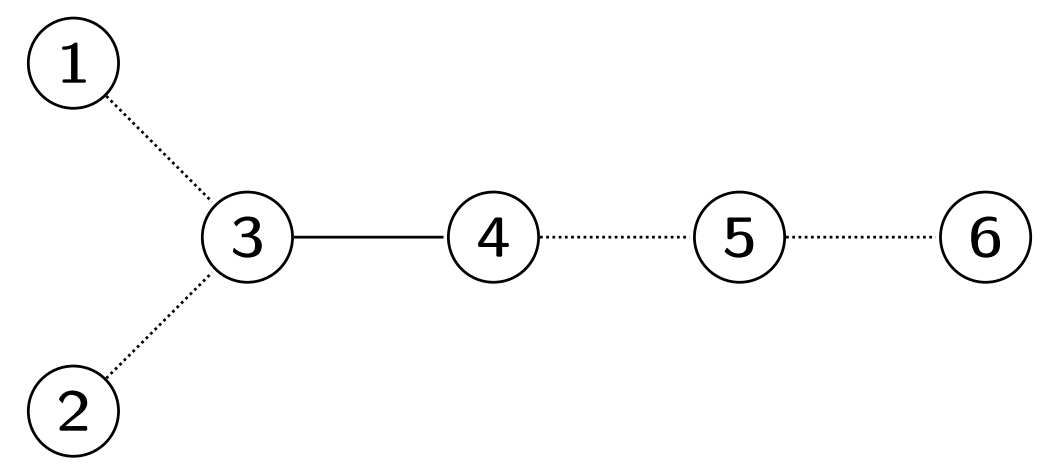

Figure 1: A network with two strategic neighborhoods.

Strategic neighborhoods play a crucial role in our analysis. They roughly correspond to weakly dependent network subunits or distinct games (although they may have nodes in common, as in the previous example). To see this, consider a counterfactual policy intervention that alters the link between nodes 5 and 6 , while keeping fixed all other structural primitives. Because $D_{45}=1, A_{45}$ is non-robust, so this policy intervention may affect the joint surplus enjoyed by 4 and 5 . As a result, their best (myopic) response may be to change their potential link. If they do so, their choice may in turn affect the links of other nodes. However, in Figure 1 no other potential links end up affected because the potential link $A_{34}$ is robust. Consequently, the subnetwork on the strategic neighborhood $\{1,2,3,4\}$ is unaffected by counterfactual manipulations of potential links involving nodes outside of this neighborhood. The following result, which corresponds to Proposition 1 of Leung (2019b), formalizes this observation, showing that the pairwise stability of the subnetwork on a strategic neighborhood $C_{i}^{+}$is invariant to the counterfactual removal of nodes $\mathcal{N}_{n} \backslash C_{i}^{+}$from the model. 


\section{LEUNG AND MOON}

Proposition 1. Under Assumption 2, for any $n \in \mathbb{N}$ and strategic neighborhood $C^{+}$,

$$
\mathcal{E}\left(r_{n}^{-1} \boldsymbol{T}_{C^{+}}, \boldsymbol{\zeta}_{C^{+}}\right)=\left\{\boldsymbol{A}_{C^{+}}: \boldsymbol{A} \in \mathcal{E}\left(r_{n}^{-1} \boldsymbol{T}_{n}, \boldsymbol{\zeta}_{n}\right)\right\} \quad \text { a.s. }
$$

The left-hand side is the set of pairwise stable networks under the counterfactual model in which the set of nodes is $C^{+}$, rather than $\mathcal{N}_{n}$. The right-hand side is the set of subnetworks on $C^{+}$of all pairwise stable networks under the model with nodes $\mathcal{N}_{n}$. The proposition asserts the two are equivalent, and in this sense, the game on $C_{i}^{+}$is separate from the game on any other strategic neighborhood. This property is unusual because if we consider an arbitrary set of nodes $H$, the subnetwork of $\boldsymbol{A}$ on $H$ would generally fail to be pairwise stable under the counterfactual that $\mathcal{N}_{n} \backslash H$ is removed from the model since nodes in $H$ would typically adjust their links in best response to the deletion of the remainder of the network. Strategic neighborhoods are the exception to this rule. Leung (2019b) uses this stability property to prove an LLN, while Leung (2020) exploits it to devise an algorithm for computing $\mathcal{E}\left(r_{n}^{-1} \boldsymbol{T}_{n}, \boldsymbol{\zeta}_{n}\right)$ in polynomial time. We utilize it to establish a CLT.

Because strategic neighborhoods roughly correspond to weakly dependent subunits, we seek to obtain conditions under which we have many such neighborhoods, which is the case when the distribution of neighborhood size has well-behaved tails. To establish the latter, we employ a well-known technique used in random graph theory for bounding the size of a network component $C_{i}$, which is to construct a branching process whose size stochastically dominates that of the component (e.g. Bollobás and Riordan, 2012). Our constructions can be found in §A.1.

The basic idea is to explore the component via breadth-first search by starting at an arbitrary node in $C_{i}$, branching to its neighbors, the neighbors of its neighbors, and so on. This is akin to growing a branching process, a model of population growth in which individuals in a given generation independently produce a random number of offspring. If the average number of offspring is less than one, the total size of the process is finite with probability one, in which case the process is called "subcritical," so the size of $C_{i}$ is asymptotically bounded. In $\S$ A.2, we utilize a tail bound on the size of subcritical branching processes to obtain tail bounds for sizes of strategic neighborhoods under Assumption 7. This is a key ingredient for verifying Assumption 5. 


\section{Normal Approximation in Large Network Models}

\subsection{Coordination}

Our final weak-dependence condition imposes a restriction on the selection mechanism. The basic issue is that if we have, for example, an entry game played in two separate markets, these markets are not independent if their selection mechanisms both depend on a common signal. In our setting, markets are strategic neighborhoods, so there exist selection mechanisms $\lambda_{n}(\cdot)$ that introduce arbitrary dependence ("coordination") between the realizations of subnetworks on different strategic neighborhoods.

Example 7. Consider Figure 1, and suppose that types and random utility shocks are realized such that the strategic neighborhood $\{1,2,3,4\}$ has two possible pairwise stable subnetworks, which we denote $\boldsymbol{A}_{1}$ and $\boldsymbol{A}_{2}$. That is, $\mathcal{E}\left(r_{n}^{-1} \boldsymbol{T}_{H}, \boldsymbol{\zeta}_{H}\right)=\left\{\boldsymbol{A}_{1}, \boldsymbol{A}_{2}\right\}$ for $H=\{1,2,3,4\}$. Further suppose there exists a unique pairwise stable subnetwork on $\{3,4,5,6\}$, so that $\left|\mathcal{E}\left(r_{n}^{-1} \boldsymbol{T}_{H^{\prime}}, \boldsymbol{\zeta}_{H^{\prime}}\right)\right|=1$ for $H^{\prime}=\{3,4,5,6\} .{ }^{5}$ Then $\mathcal{E}\left(r_{n}^{-1} \boldsymbol{T}_{n}, \boldsymbol{\zeta}_{n}\right)=\left\{\boldsymbol{A}_{1}{ }^{\prime}, \boldsymbol{A}_{2}^{\prime}\right\}$, where $\boldsymbol{A}_{1}^{\prime}\left(\boldsymbol{A}_{2}^{\prime}\right)$ denotes the equilibrium in which the subnetwork on $\{1,2,3,4\}$ is $\boldsymbol{A}_{1}\left(\boldsymbol{A}_{2}\right)$. Consider a selection mechanism $\lambda_{n}(\cdot)$ that chooses $\boldsymbol{A}_{1}^{\prime}$ if $Z_{5}$ lies in a certain set and otherwise chooses $\boldsymbol{A}_{2}$. Then the realization of all potential links in the network depends on $Z_{5}$, so potential links generally fail to be weakly dependent. The effective problem is that all nodes in the network "coordinate" on the same public signal $Z_{5}$.

Our next assumption rules out coordination of this type. It requires a form of "decentralization" under which a given strategic neighborhood $C^{+}$selects its pairwise stable subnetwork on the basis of the types and random-utility shocks of nodes in $\mathrm{C}^{+}$alone. Intuitively, strategic neighborhoods correspond to distinct games, and equilibrium selection needs to be independent across games that have no nodes in common (those with nodes in common, such as the two in Figure 1, are naturally allowed to be dependent).

As with our Assumption 7, Sheng (2020) does not impose such an assumption because she considers a setting with many small independent networks. However, this necessarily implies that equilibrium selection is independent across network observations. Our condition is the analog for the single-network setting.

\footnotetext{
${ }^{5}$ Since $A_{34}$, for example, is a robust link, its realization is necessarily the same under all such subnetworks.
} 


\section{LEUNG AND MOON}

To formally state the assumption, let $\left.\lambda_{n}\left(r_{n}^{-1} \boldsymbol{T}_{n}, \boldsymbol{\zeta}_{n}\right)\right|_{H}$ be the restriction of the range of $\lambda_{n}(\cdot)$ to networks on $H$. For example, under Assumption $3, \lambda_{n}\left(r_{n}^{-1} \boldsymbol{T}_{n}, \boldsymbol{\zeta}_{n}\right)=$ $\boldsymbol{A}$, so $\left.\lambda_{n}\left(r_{n}^{-1} \boldsymbol{T}_{n}, \boldsymbol{\zeta}_{n}\right)\right|_{H}=\boldsymbol{A}_{H}$.

Assumption 8 (Decentralized Selection). For any $n \in \mathbb{N}, i \in \mathcal{N}_{n}$, and $H=C_{i}^{+}$, $\left.\lambda_{n}\left(r_{n}^{-1} \boldsymbol{T}_{n}, \boldsymbol{\zeta}_{n}\right)\right|_{H}=\lambda_{|H|}\left(r_{n}^{-1} \boldsymbol{T}_{H}, \boldsymbol{\zeta}_{H}\right)$.

It is important to understand the difference between the left- and right-hand sides of the equality. On the left-hand side, we have an $n$-node model in which the selection mechanism chooses network $\boldsymbol{A}$. We then take the subnetwork $\boldsymbol{A}_{H}$ on $i$ 's strategic neighborhood $H=C_{i}^{+}$. On the right-hand side, we instead counterfactually remove nodes in $\mathcal{N}_{n} \backslash H$ from the model while fixing all other structural primitives, so that we have a $|H|$-node model, and the selection mechanism $\lambda_{|H|}(\cdot)$ chooses some network $\lambda_{|H|}\left(r_{n}^{-1} \boldsymbol{T}_{H}, \boldsymbol{\zeta}_{H}\right)$. For any arbitrary $H \subseteq \mathcal{N}_{n}$, it will typically not be the case that $\boldsymbol{A}_{H}$ (the realized subnetwork on $H$ ) equals $\lambda_{|H|}\left(r_{n}^{-1} \boldsymbol{T}_{H}, \boldsymbol{\zeta}_{H}\right)$ (the counterfactual subnetwork). However, if $H$ is a strategic neighborhood, then as discussed in the previous subsection, the set of pairwise stable subnetworks on $H$, namely $\mathcal{E}\left(r_{n}^{-1} \boldsymbol{T}_{H}, \boldsymbol{\zeta}_{H}\right)$, is equivalent to the set subnetworks on $H$ of pairwise stable networks on $\mathcal{N}_{n}$, namely $\left\{\boldsymbol{A}_{H}: \boldsymbol{A} \in \mathcal{E}\left(r_{n}^{-1} \boldsymbol{T}_{n}, \boldsymbol{\zeta}_{n}\right)\right\}$. It is therefore well-defined to impose Assumption 8.

Assumption 8 corresponds to Assumption 7 of Leung (2019b). The assumption rules out selection mechanisms in which all nodes coordinate on the basis of a common signal, such as a single node's type. In the special case where there exists a unique equilibrium on $\mathcal{N}_{n}$, for instance if there are no strategic interactions, the assumption holds trivially. More generally, the condition is satisfied by variants of myopic best-response dynamics, which are the most widely used selection mechanisms in the theoretical and econometric literature on dynamic network formation (e.g. Jackson, 2010; Mele, 2017; Leung, 2019b). A simple example of myopic best-response dynamics is the following.

Example 8. Arbitrarily order all pairs of nodes, and begin with the empty network $\boldsymbol{A}_{0}$ (for example). For the first pair of nodes $(i, j)$, update $\boldsymbol{A}_{0}$ by setting $A_{i j}=$ $\mathbf{1}\left\{V\left(r_{n}^{-1}\left\|X_{i}-X_{j}\right\|, S_{i j}, Z_{i}, Z_{j}, \zeta_{i j}\right)>0\right\}$, where $S_{i j}=S_{n}\left(i, j, r_{n}^{-1} \boldsymbol{T}_{n}, \boldsymbol{A}_{0}\right)$, the network

statistics evaluated at the prior network. Repeat for all pairs of nodes to obtain $\boldsymbol{A}_{1}$, and then repeat this process until convergence. 


\section{Normal Approximation in Large Network Models}

These dynamics may be run on the entire set of nodes $\mathcal{N}_{n}$ to obtain an equilibrium. Equivalently, they may be run separately on each strategic neighborhood, after which we take their "Cartesian product" to obtain the same equilibrium (Leung, 2020).

\subsection{Main Results}

The last assumption we require is a regularity condition.

Assumption 9 (Regularity). Either $p_{r}\left(X_{1}, Z_{1} ; X_{2}, Z_{2}\right)=0$ a.s. for any $r>0$ in a neighborhood of zero, or

$$
\inf _{(x, z) \in \operatorname{supp}\left(X_{1}, Z_{1}\right)} \lim _{n \rightarrow \infty} n \mathbf{E}\left[p_{r_{n}}\left(X_{1}, Z_{1} ; X_{2}, Z_{2}\right) \mid X_{1}=x, Z_{1}=z\right]>0 .
$$

The choice of nodes 1 and 2 is arbitrary because nodes are exchangeable. The case $p_{r}\left(X_{1}, Z_{1} ; X_{2}, Z_{2}\right)=0$ corresponds to a model without strategic interactions, which is only mentioned for completeness. For the more interesting case, the assumption essentially requires that strategic interactions are sufficiently nontrivial for all nodes in the sense that $p_{r_{n}}\left(X_{1}, Z_{1} ; X_{2}, Z_{2}\right)$ is at least order $n^{-1}$, which is a very mild requirement. It is typically satisfied because the two probabilities in (15) are upper and lower bounds on the probability of link formation, and the latter is order $n^{-1}$ under sparsity by (6).

Example 9. Consider Example 6, and recall the notation used there. Following the derivation in that example and (6),

$$
\begin{aligned}
n \mathbf{E}\left[p_{r}\left(X_{1}, Z_{1} ; X_{2}, Z_{2}\right) \mid X_{1}\right. & \left.=x, Z_{1}=z\right] \\
& =2 n r^{2} \pi \int_{0}^{1} z\left[\Phi\left(\theta_{1}+\bar{S}^{\prime} \theta_{2}-\theta_{3} z^{2}\right)-\Phi\left(\theta_{1}-\theta_{3} z^{2}\right)\right] \mathrm{d} z .
\end{aligned}
$$

If $\theta_{2}=0$, this corresponds to the case $p_{r}\left(X_{1}, Z_{1} ; X_{2}, Z_{2}\right)=0$ a.s. Otherwise, Assumption 9 holds if $\bar{S} \neq \mathbf{0}$ since $n r_{n}^{2}=\kappa>0$ by (5).

Theorem 2. Assumptions 1-4 and 7-9 imply Assumption 5.

Proof. See $\S$ A. We provide a proof sketch below. 


\section{LEUNG AND MOON}

Theorems 1 and 2 immediately imply the following CLT for the model in $§ 2$.

Corollary 1. Under Assumptions 1-4 and 6-9, $\sup _{n}\left\|\boldsymbol{\Sigma}_{n}\right\|<\infty$, and if additionally $\liminf _{n \rightarrow \infty} \lambda_{\min }\left(\boldsymbol{\Sigma}_{n}\right)>0$, then (12) holds.

At this level of generality, these conditions are close to as primitive as possible, but we can derive conditions that are more primitive still with additional structure. We illustrate with Example 6.

Corollary 2. Consider the network formation model

$$
V\left(r_{n}^{-1}\left\|X_{i}-X_{j}\right\|, S_{i j}, Z_{i}, Z_{j}, \zeta_{i j}\right)=\theta_{1}+S_{i j}^{\prime} \theta_{2}-\theta_{3}\left(r_{n}^{-1}\left\|X_{i}-X_{j}\right\|\right)^{2}+\zeta_{i j},
$$

where $r_{n}$ is defined in (5), $\left\{X_{i}\right\} \stackrel{\text { iid }}{\sim} \mathcal{U}\left([0,1]^{2}\right),\left\{\zeta_{i j}\right\} \stackrel{\text { iid }}{\sim} \mathcal{N}(0,1)$, and there exists a vector of strictly positive constants $\bar{S}$ such that $\left|S_{i j}^{\prime} \theta_{2}\right| \leqslant \bar{S}^{\prime} \theta_{2}$ a.s. for any $n$. Assume the following.

(a) For $h(x)=x \Phi(x)+\phi(x)$, where $\Phi(\cdot)$ and $\phi(\cdot)$ are the normal CDF and PDF, respectively,

$$
\frac{\kappa \pi}{\theta_{3}}\left(h\left(\theta_{1}+\bar{S}^{\prime} \theta_{2}\right)-h\left(\theta_{1}\right)\right)<1
$$

(Example 6 for an economic interpretation.)

(b) Externalities, as determined by $S_{i j}$, are local in the sense of Assumption 2.

(c) The equilibrium is selected via myopic best-response dynamics as in Example 8.

If $\sum_{i=1}^{n} \psi_{i}\left(\mathcal{N}_{n}\right)$ is a vector of subnetwork counts (Example 4), then (12) holds.

PROOF. We verify the conditions of Corollary 1. Assumption 1 holds because

$$
\bar{\Phi}_{\zeta}\left(\bar{V}^{-1}(\delta, 0)\right)=\bar{\Phi}_{\zeta}\left(\theta_{3} \delta^{2}-\theta_{1}-\bar{S}^{\prime} \theta_{2}\right)
$$

which decays to zero exponentially with $\delta$ since $\bar{\Phi}_{\zeta}$ is the complementary CDF of the standard normal distribution. Assumption 2 holds by (b). Assumptions 3 and 8 hold by (c). Assumption 4 holds because we consider subnetwork counts (see Example 4). Assumption 6 follows from Proposition B.1. Assumption 7 follows from (a) (see Example 6). Finally, Assumption 9 holds because $\bar{S} \neq \mathbf{0}$ (see Example 9). 


\section{Normal Approximation in Large Network Models}

\subsection{Method of Proof}

We next detail the method of proof for Theorem 2, which has been applied to other network models, including games on networks (Leung, 2019a) and dynamic network formation (Leung and Moon, 2019). We illustrate the idea for the case of the average degree moment where $\psi_{i}\left(\mathcal{N}_{n}\right)=\sum_{j=1}^{n} A_{i j}$. The goal is to construct an upper bound on the radius of stabilization that has exponential tails.

Step 1. Recalling (9), we construct a set of nodes $J_{i} \subseteq \mathcal{N}_{n}$ positioned near $i$ such that

$$
\psi_{i}\left(\mathcal{N}_{n}\right)=\psi_{i}\left(J_{i}\right)
$$

That is, $i$ 's node statistic is invariant to the counterfactual removal of nodes outside of $J_{i}$. A trivial choice is $J_{i}=\mathcal{N}_{n}$, but the challenge is to find a set that is relatively small so that step two below is possible. Given such a set, the radius of stabilization is upper bounded by the largest neighborhood about $i$ containing $J_{i}$ :

$$
\boldsymbol{R}_{i}\left(n, r_{n}\right) \leqslant \tilde{\boldsymbol{R}}_{i}\left(n, r_{n}\right) \equiv \max _{j \in J_{i}} r_{n}^{-1}\left\|X_{i}-X_{j}\right\|
$$

In the case of degree, which is simply the 1-neighborhood size, a naive choice of $J_{i}$ might be $i$ 's 1-neighborhood $\mathcal{N}_{\boldsymbol{A}}(i, 1)$. To see why this does not generally satisfy (18), consider node 6 in Figure 1, whose 1-neighborhood is $\{5,6\}$. Due to strategic interactions and the fact that $D_{56}=1$ (their potential link is non-robust), $A_{56}$ may differ under the counterfactual that removes node $4 \in \mathcal{N}_{n} \backslash\{5,6\}$ from the model.

A better choice is $J_{i}=C_{i}^{+}$, which does satisfy (18). To see this in Figure 1, notice that $C_{6}^{+}=\{3,4,5,6\}$. Under Assumption 8, the equilibrium subnetwork on $C_{6}^{+}$is invariant to the removal of $\{1,2\}$. Since $C_{i}^{+}$necessarily contains $i$ 's 1-neighborhood, (18) holds.

Step 2. We show that $\left|J_{i}\right|$ has exponential tails. First, §A.1 establishes that $\left|J_{i}\right|$ is stochastically bounded above by the size of a certain branching process when $J_{i}$ consists of components (e.g. $C_{i}$ ) and $K$-neighborhoods. Then Lemma A.3 shows that the size of the branching process has exponential tails using Assumptions 7 and 9 and auxiliary lemmas in $\S \mathrm{SA} .2$.

In the case of average degree, $J_{i}=C_{i}^{+}$is the union of a component of a network $\boldsymbol{D}$ and certain 1-neighborhoods due to the addition of robust links in the definition 


\section{LEUNG AND MOON}

of $C_{i}^{+}$. For more general node statistics satisfying Assumption 4, we construct $J_{i}$ in (A.2) as a union of components and $K$-neighborhoods. Leung (2019a) provides a different construction of $J_{i}$ for games on networks and Leung and Moon (2019) for dynamic network formation, but all constructions are unions of these same objects, so the auxiliary lemmas in $§ S A .2$ may be applied to obtain the desired tail bounds.

Step 3. We translate the tail bound for $\left|J_{i}\right|$ into one for $\tilde{\boldsymbol{R}}_{i}\left(n, r_{n}\right)$. Intuitively, if $\left|J_{i}\right|$ is small, then so will be $\tilde{\boldsymbol{R}}_{i}\left(n, r_{n}\right)$ since nodes are homophilous in positions (Assumption 1), so each $j \in J_{i}$ will typically be close to $i$ in terms of distance $r_{n}^{-1} \| X_{i}-$ $X_{j} \|$. Lemma A.4 provides the formal argument.

\section{Applications to Inference}

We close with a discussion of inference procedures justified by Theorem 2 .

\subsection{Inference on Network Moments}

Define $\mu_{0}=\mathbf{E}\left[\psi_{1}\left(\mathcal{N}_{n}\right)\right]$ and $\hat{\mu}=n^{-1} \sum_{i=1}^{n} \psi_{i}\left(\mathcal{N}_{n}\right)$. Consider testing the null hypothesis

$$
H_{0}: \mu_{0}=\mu \text {. }
$$

This is empirically relevant for the reporting of stylized facts in the networks literature. Such facts are obtained by computing various network summary statistics, which are seldom accompanied by formal uncertainty quantification due to a lack of available methods. Leung (2022) discusses two particular examples: testing for nontrivial clustering (his §3.3) and testing for a power law degree distribution (his $\S 3.4)$. Both cases fall within the setup here. We next discuss two tests available in the literature that are justified by our CLT. Confidence regions for $\mu_{0}$ can be obtained by test inversion.

Single large network. If the sample consists of a single network, we may apply the resampling procedure proposed by Leung (2022). Let $\alpha$ be the desired level of the test, $R_{n}=(n / 2)^{4 / 3}$ rounded to the nearest integer, $\Pi$ be the set of all bijections

(permutation functions) on $\{1, \ldots, n\}$, and $\pi=\left(\pi_{r}\right)_{r=1}^{R_{n}}$ be i.i.d. uniform draws from П. Let $\hat{\boldsymbol{V}}=n^{-1} \sum_{i=1}^{n}\left(\psi_{i}\left(\mathcal{N}_{n}\right)-\hat{\mu}\right)\left(\psi_{i}\left(\mathcal{N}_{n}\right)-\hat{\mu}\right)^{\prime}$, the sample variance. Define the test 


\section{Normal Approximation in Large Network Models}

statistic

$$
T_{U}(\mu ; \pi)=\frac{1}{\sqrt{d_{\psi} R_{n}}} \sum_{r=1}^{R_{n}}\left(\psi_{\pi_{r}(1)}\left(\mathcal{N}_{n}\right)-\mu\right)^{\prime} \hat{\boldsymbol{V}}^{-1}\left(\psi_{\pi_{r}(2)}\left(\mathcal{N}_{n}\right)-\mu\right)
$$

recalling that $d_{\psi}$ is the dimension of the range of $\psi(\cdot)$. Let $z_{1-\alpha}$ be the $1-\alpha$ quantile of the standard normal distribution. The test rejects if and only if

$$
T_{U}(\mu ; \pi)>z_{1-\alpha}
$$

Theorem A.1 of Leung (2022) provides conditions under which the test is asymptotically level $\alpha$ under $H_{0}$. The main assumption that needs to be verified is $\sqrt{n}$ consistency of $\hat{\mu}$, which is a consequence of our Theorem $2 .^{6}$

For intuition on the importance of $\sqrt{n}$-consistency, consider a simpler test statistic studied by Leung (2019a):

$$
\tilde{T}_{M}(\mu ; \pi)=\frac{1}{\sqrt{R_{n}^{M}}} \sum_{r=1}^{R_{n}^{M}} \hat{\boldsymbol{V}}^{-1 / 2}\left(\psi_{\pi_{r}(1)}\left(\mathcal{N}_{n}\right)-\mu\right)
$$

for $R_{n}^{M}=\sqrt{n}$ rounded to the nearest integer. To understand its asymptotic behavior, we add and subtract its conditional mean:

$$
\begin{aligned}
\frac{1}{\sqrt{R_{n}^{M}}} \sum_{r=1}^{R_{n}^{M}} \hat{\boldsymbol{V}}^{-1 / 2}\left(\psi_{\pi_{r}(1)}\left(\mathcal{N}_{n}\right)\right. & \left.-\mathbf{E}\left[\psi_{\pi_{r}(1)}\left(\mathcal{N}_{n}\right) \mid\left\{\psi_{i}\left(\mathcal{N}_{n}\right)\right\}_{i=1}^{n}\right]\right) \\
& +\frac{1}{\sqrt{R_{n}^{M}}} \sum_{r=1}^{R_{n}^{M}} \hat{\boldsymbol{V}}^{-1 / 2}\left(\mathbf{E}\left[\psi_{\pi_{r}(1)}\left(\mathcal{N}_{n}\right) \mid\left\{\psi_{i}\left(\mathcal{N}_{n}\right)\right\}_{i=1}^{n}\right]-\mu\right) .
\end{aligned}
$$

Conditional on the data $\left\{\psi_{i}\left(\mathcal{N}_{n}\right)\right\}_{i=1}^{n}$, the permutations $\pi_{r}$ are independent, so the first term is an average of $R_{n}^{M}$ conditionally independent observations and therefore obeys a CLT. The second is a bias term that can be shown to equal $\left(R_{n}^{M} / n\right)^{1 / 2} \hat{\boldsymbol{V}}^{-1 / 2} \sqrt{n}(\hat{\mu}-\mu)$. Hence, under $H_{0}$ and $\sqrt{n}$-consistency, this is order $\left(R_{n}^{M} / n\right)^{1 / 2}=o(1)$.

Multiple large networks. A drawback of the previous procedure is that it is inefficient, having a rate of convergence slower than $\sqrt{n}$ since $R_{n}^{M}=o(n)$. If the sample consists of multiple independent large networks, then more powerful methods

\footnotetext{
${ }^{6}$ The LLN in Leung (2019b) does not deliver a rate of convergence.
} 


\section{LEUNG AND MOON}

are available from the cluster-robust inference literature. Consider a sequence of $L$ independent networks indexed by $n$, where each network $\ell=1, \ldots, L$ has size $n_{\ell}$ satisfying $n_{\ell} / n \rightarrow c_{\ell} \in(0, \infty)$ as $n \rightarrow \infty$. Let $\hat{\mu}^{\ell}=n_{\ell}^{-1} \sum_{i=1}^{n_{\ell}} \psi_{i}\left(\mathcal{N}_{n_{\ell}}\right)$, the network moment computed on network $\ell$. Assume there exists a universal population moment $\mu_{0}$ such that $\mathbf{E}\left[\hat{\mu}^{\ell}\right]=\mu_{0}+o\left(n^{-1 / 2}\right)$ for all $\ell$. We seek to test the null $H_{0}: \mu_{0}=\mu$.

We consider the randomization test proposed by Canay et al. (2017). For $S_{n, \ell}=$ $\sqrt{n_{\ell}}\left(\hat{\mu}^{\ell}-\mu\right)$ and $S_{n}=\left(S_{n, \ell}\right)_{\ell=1}^{L}$, define the Wald statistic

$$
T\left(S_{n}\right)=\left(\frac{1}{\sqrt{L}} \sum_{\ell=1}^{L} S_{n, \ell}^{\prime}\right)\left(\frac{1}{L} \sum_{\ell=1}^{L} S_{n, \ell} S_{n, \ell}^{\prime}\right)^{-1}\left(\frac{1}{\sqrt{L}} \sum_{\ell=1}^{L} S_{n, \ell}\right)
$$

We obtain critical values from the randomization distribution $\left\{T\left(\pi S_{n}\right): \pi \in\{-1,1\}^{L}\right\}$ where $\pi S_{n}=\left(\pi_{\ell} S_{n, \ell}\right)_{\ell=1}^{L}$ for $\pi=\left(\pi_{\ell}\right)_{\ell=1}^{L}$. Let $\alpha$ be the desired level of the test, $q=2^{L}(1-\alpha)$ rounded up to the nearest integer, and $c_{L, 1-\alpha}$ be the $q$ th largest value of $\left\{T\left(\pi S_{n}\right): \pi \in\{-1,1\}^{L}\right\}$. The test rejects if and only if

$$
T\left(S_{n}\right)>c_{L, 1-\alpha}
$$

The test is asymptotically level $\alpha$ if the limit distribution of the vector of network moments is asymptotically normal, which is a consequence of our Theorem 2. Intuitively, under $H_{0}$ and asymptotic normality, $\left\{S_{n, \ell}\right\}_{\ell=1}^{L}$ are approximately independent draws from a mean-zero normal distribution. Hence, multiplying these draws by \pm 1 does not change the asymptotic distribution of $T\left(S_{n}\right)$, which is the key justification for the validity of randomization tests (see $\S 2$ of Canay et al., 2017, for a review of such tests).

\subsection{Structural Inference}

We revisit Example 4, which concerns structural inference using moment inequalities proposed by Sheng (2020). Recalling the setup there, let $\theta_{0}$ denote the true parameters of $V(\cdot)$. We maintain the assumptions of Corollary 1 in order to apply our CLT. To test the hypothesis $H_{0}: \theta_{0}=\theta$, we test the moment inequality

$$
\mu_{0} \equiv \mathbf{E}\left[G_{1}-H_{1}(\theta)\right] \leqslant \mathbf{0}
$$




\section{Normal Approximation in Large Network Models}

using the moment inequality test proposed by Leung (2022).

Returning to the single-network setting, let $\mu_{k}$ be the $k$ th component of $\mu_{0}$, $\psi_{i k}\left(\mathcal{N}_{n}\right)$ the $k$ th component of $\psi_{i}\left(\mathcal{N}_{n}\right)$, and $T_{U, k}\left(\mu_{k} ; \pi\right)$ the U-type statistic defined in the previous subsection but computed with scalar data $\left\{\psi_{i k}\left(\mathcal{N}_{n}\right)\right\}_{i=1}^{n}$. Also let $\hat{\mu}_{k}$ be the $k$ th component of $\hat{\mu}$ and $\hat{\boldsymbol{V}}_{k k}$ the $k$ th diagonal entry of $\hat{\boldsymbol{V}}$. Define the test statistic

$$
\begin{aligned}
& Q_{n}(\pi)=\max _{1 \leqslant k \leqslant d_{\psi}}\left\{T_{U, k}(0 ; \pi)-\mu_{k}^{*} \mathbf{1}\left\{\hat{\mu}_{k}<0\right\}\right\}, \quad \text { where } \\
& \mu_{k}^{*}=\hat{\mu}_{k} \hat{\boldsymbol{V}}_{k k}^{-1} \frac{1}{\sqrt{d_{\psi} R_{n}}} \sum_{r=1}^{R_{n}}\left(\psi_{\pi_{r}(1), k}\left(\mathcal{N}_{n}\right)+\psi_{\pi_{r}(2), k}\left(\mathcal{N}_{n}\right)\right)-\sqrt{\frac{R_{n}}{d_{\psi}}} \hat{\boldsymbol{V}}_{k k}^{-1} \hat{\mu}_{k}^{2} .
\end{aligned}
$$

Let $\tilde{\pi}_{1}, \ldots, \tilde{\pi}_{L}$ be i.i.d. with the same distribution as $\pi$. Let $q=L(1-\alpha)$ rounded up to the nearest integer and $c_{L, 1-\alpha}$ be $q$ th largest value of $\left\{\max _{1 \leqslant k \leqslant d_{\psi}} T_{U, k}\left(\hat{\mu}_{k} ; \tilde{\pi}_{\ell}\right)\right\}_{\ell=1}^{L}$. The test rejects if and only if

$$
Q_{n}(\pi)>c_{L, 1-\alpha} .
$$

Theorem 2 of Leung (2022) provides conditions under which the test is asymptotically level $\alpha$ under $H_{0}$. The main assumption that needs to be verified is $\sqrt{n}$-consistency of $\hat{\mu}$, which is a consequence of Theorem 2 .

In order to construct more powerful tests, we require either a consistent estimate of $\boldsymbol{\Sigma}_{n}$ or a valid resampling procedure, topics we leave to future research.

\section{Conclusion}

This paper develops a large-sample theory for a model of network formation with strategic interactions and homophilous agents when the data consists of a small sample of large networks or possibly a single network. We prove a general CLT under a high-level weak-dependence condition and provide a general methodology for its verification. We apply the methodology to obtain primitive conditions for a CLT for moments of pairwise stable networks. The methodology can be more broadly applied to dynamic network formation (Leung and Moon, 2019) and models of discrete choice with social interactions (Leung, 2019a). There are several important directions for future work. Concentration inequalities for stabilization would be useful, for example, for deriving lower-level conditions for uniform convergence of nonparametric or high-dimensional estimators using network data. It is also of interest to develop effi- 


\section{LEUNG AND MOON}

cient alternatives to the inference procedures in $\S 5$ that only require a single network observation.

\section{A Proof of Theorem 2}

The results in this section make use of the following notation. Because we are verifying Assumption 5, we consider a model with $m$ nodes and sparsity parameter $r_{n}$ with $m$ potentially different from $n$. For $m, n \in \mathbb{N}$, we let $\boldsymbol{A}=\lambda\left(r_{n}^{-1} \boldsymbol{T}_{m}, \boldsymbol{\zeta}_{m}\right)$, per Assumption 3. For the same primitives $\left(r_{n}^{-1} \boldsymbol{T}_{m}, \boldsymbol{\zeta}_{m}\right)$, let $\boldsymbol{D} \equiv \boldsymbol{D}(m)$ be the network on $\mathcal{N}_{m}$ with $i j$ th entry $D_{i j}$ defined in (16). Let $C_{i} \equiv C_{i}(m)$ be $i$ 's component in $\boldsymbol{D}(m)$, and define $C_{i}^{+} \equiv C_{i}^{+}(m)$ as in (17). Define $\boldsymbol{M} \equiv \boldsymbol{M}(m)$ as the $m \times m$ adjacency matrix with all $M_{i i}=0$ and $i j$ th entry

$$
M_{i j}=1\left\{\sup _{s} V\left(r_{n}^{-1}\left\|X_{i}-X_{j}\right\|, s, Z_{i}, Z_{j}, \zeta_{i j}\right)>0\right\} .
$$

Finally, for any $J \subseteq \mathcal{N}_{m}$ and $i \in J$, abbreviate

$$
\psi_{i}(J) \equiv \psi\left(i, r_{n}^{-1} \boldsymbol{T}_{J}, \boldsymbol{\zeta}_{J}, \lambda_{|J|}\right) \equiv \psi\left(i, r_{n}^{-1} \boldsymbol{T}_{J}, \boldsymbol{\zeta}_{J}, \lambda_{|J|}\left(r_{n}^{-1} \boldsymbol{T}_{J}, \boldsymbol{\zeta}_{J}\right)\right)
$$

Proof of Theorem 2. Lemma A.1 constructs, for any $i \in \mathcal{N}_{m}$, a set $J_{i}$ satisfying (18). In $\S \mathrm{A} .1$, we construct a branching process $\mathfrak{X}_{r_{n}}^{H}\left(X_{i}, Z_{i} ; K\right)$, defined in (A.7), whose size stochastically dominates $\left|J_{i}\right|$ (Lemma A.2). Lemma SA.2.3 derives an exponential tail bound on the size of the branching process, which is used to establish a corresponding tail bound for $\left|J_{i}\right|$ in Lemma A.3. Finally, Lemma A.4 translates this to a uniform exponential tail bound on $\tilde{\boldsymbol{R}}_{i}\left(m, r_{n}\right)$ defined in (19). Since this is an upper bound on the radius of stabilization, we have verified Assumption 5.

The following lemma corresponds to Lemma 1 of Leung (2019b), which constructs $J_{i}$ satisfying (18). Recall that $\mathcal{N}_{\boldsymbol{M}}(i, K)$ is $i$ 's $K$-neighborhood in the network $\boldsymbol{M}$.

Lemma A.1. Under Assumptions 2, 4, and 8, for any $m, n \in \mathbb{N}$ and $i \in \mathcal{N}_{m}$, $\psi_{i}\left(\mathcal{N}_{m}\right)=\psi_{i}\left(J_{i}\right)$ a.s. for

$$
J_{i} \equiv J_{i}(m)=\bigcup\left\{C_{j}^{+}(m): j \in \mathcal{N}_{\boldsymbol{M}(m)}(i, K)\right\}
$$




\section{Normal Approximation in Large Network Models}

\section{A.1 Branching Process Bound}

We next define branching processes used to stochastically bound the sizes of $C_{i}^{+}$ and $\mathcal{N}_{\boldsymbol{M}}(i, K)$, defined in the previous subsection. These are used to construct a process whose size stochastically dominates $\left|J_{i}\right|$ defined in (A.2). The use of branching processes to bound component and neighborhood sizes is a well-known technique in random graph theory (e.g. Bollobás and Riordan, 2012).

Let $x \in \mathbb{R}^{d}, z \in \mathbb{R}^{d_{z}}, r>0$, and $\bar{f}=\sup _{x \in \mathbb{R}^{d}} f(x)$. Recall the definition of $\Phi^{*}(\cdot)$ from Assumption 7 and $p_{1}\left(x, z ; x^{\prime}, z^{\prime}\right)$ from (15). Denote by $\mathfrak{X}_{r}^{D}(x, z)$ the multi-type Galton-Walton branching process with type space $\mathbb{R}^{d} \times \mathbb{R}^{d_{z}}$ starting at a particle of type $(x, z)$ with the following two properties (see e.g. Bollobás et al., 2007, §2.1). First, each particle of type $\left(x^{\prime}, z^{\prime}\right)$ is replaced in the next generation by a set of particles (its "offspring") distributed as a Poisson point process on $\mathbb{R}^{d} \times \mathbb{R}^{d_{z}}$ with intensity

$$
\mathrm{d} \varphi_{r}\left(x^{\prime}, z^{\prime} ; x^{\prime \prime}, z^{\prime \prime}\right)=\kappa \bar{f}(1+r) p_{1}\left(x^{\prime}, z^{\prime} ; x^{\prime \prime}, z^{\prime \prime}\right) \mathrm{d} \Phi^{*}\left(z^{\prime \prime}\right) \mathrm{d} x^{\prime \prime} .
$$

Second, conditional on particle types at generation $t-1$, the Poisson point processes that create the particles of generation $t$ are independent across generation $t-1$ particles. We can interpret $\mathfrak{X}_{r}^{D}(x, z)$ as a tree network. The root of the tree, corresponding to the first generation of the process, consists of a single node (particle) of type $(x, z)$. Its neighbors are its offspring, which constitute the second generation and are nodes with types distributed according to the Poisson point process described above. Then conditional on the types of the second generation, each node in the second generation of type $\left(x^{\prime}, z^{\prime}\right)$ independently generates neighbors with types distributed as the Poisson point process above. These constitute the third generation. The process is repeated indefinitely. Let $\left|\mathfrak{X}_{r}^{D}(x, z)\right|$ denote the number of particles ultimately generated, which may be infinite. We write $\left(x^{\prime}, z^{\prime}\right) \in \mathfrak{X}_{r}^{D}(x, z)$ to mean that $\left(x^{\prime}, z^{\prime}\right)$ is a type of a particle generated at some point in the process, viewing $\mathfrak{X}_{r}^{D}(x, z)$ as the set of all such particles.

We next define a "fixed-depth" branching process, which is required to terminate after a given number of generations. Recalling the definitions in Assumption 1, let

$$
\left.\bar{p}_{r}\left(x ; x^{\prime}\right)=\mathbf{P}\left(\bar{V}\left(r^{-1}\left\|x-x^{\prime}\right\|, \zeta_{i j}\right)>0\right\}\right) .
$$

Let $\mathfrak{X}_{r}^{M}(x, z ; K)$ denote the branching process on $\mathbb{R}^{d} \times \mathbb{R}^{d_{z}}$ that terminates after $K+1$ 


\section{LEUNG AND MOON}

generations, starting at a particle of type $(x, z)$, with the following two properties. First, any particle of type $\left(x^{\prime}, z^{\prime}\right)$ is replaced in the next generation by offspring with types distributed as a Poisson point process on $\mathbb{R}^{d} \times \mathbb{R}^{d_{z}}$ with intensity

$$
\mathrm{d} \bar{\varphi}_{r}\left(x^{\prime}, z^{\prime} ; x^{\prime \prime}, z^{\prime \prime}\right)=\kappa \bar{f}(1+r) \bar{p}_{1}\left(x^{\prime} ; x^{\prime \prime}\right) \mathrm{d} \Phi^{*}\left(z^{\prime \prime}\right) \mathrm{d} x^{\prime \prime}
$$

Second, conditional on the types of generation $t-1$, the point processes that constitute generation $t$ are independent. Thus, the process is generated the same way as $\mathfrak{X}_{r}^{D}(x, z)$, except the intensity measure is different, and once the $(K+1)$-th generation is born, no further offspring are generated. Define $\left|\mathfrak{X}_{r}^{M}(x, z)\right|$ and $\left(x^{\prime}, z^{\prime}\right) \in \mathfrak{X}_{r}^{M}(x, z)$ as we did for $\mathfrak{X}_{r}^{D}(x, z)$.

We use $\left|\mathfrak{X}_{r_{n}}^{D}\left(X_{i}, Z_{i}\right)\right|$ and $\left|\mathfrak{X}_{r_{n}}^{M}\left(X_{i}, Z_{i} ; K\right)\right|$ to stochastically bound $\left|C_{i}\right|$ and $\left|\mathcal{N}_{\boldsymbol{M}}(i, K)\right|$, respectively. Intuitively, the number of offspring of any particle stochastically dominates the degree of a node in the associated network $\boldsymbol{D}$ or $\boldsymbol{M}$.

Lemma A.2. Under Assumptions 1, 7, and 9, for $m, n$ sufficiently large and any $i \in$ $\mathcal{N}_{m},\left|C_{i}(m)\right|$ and $\left|\mathcal{N}_{M(m)}(i, K)\right|$ are respectively stochastically dominated by $\left|\mathfrak{X}_{r_{n}}^{D}\left(X_{i}, Z_{i}\right)\right|$ and $\left|\mathfrak{X}_{r_{n}}^{M}\left(X_{i}, Z_{i} ; K\right)\right|$ conditional on $\left(X_{i}, Z_{i}\right)$. The same result holds if we replace $m$ with $N_{n}+1$.

Proof. The result for $C_{i}(m)$ follows from Lemma SA.3.1 of Leung (2020). The key argument in the lemma is that we can stochastically bound the degree distribution of any node $j$ in $\boldsymbol{D}(m)$, which is conditionally binomial, with the Poisson point process with intensity (A.3). A similar argument establishes the result for $m=N_{n}+1$, which is even simpler since the degree distribution in this case is exactly Poisson. The corresponding result for $\mathcal{N}_{\boldsymbol{M}(m)}(i, K)$ follows from the same arguments and only requires Assumption 1. The proof for this case is even simpler because we terminate the branching process after $K+1$ generations rather than branching indefinitely.

With this result in hand, we may stochastically bound $\left|J_{i}\right|$ as follows. Let $C_{i}^{*} \equiv$ $C_{i}^{*}(m)=C_{i}(m) \cup \cup_{j \in C_{i}(m)} \mathcal{N}_{\boldsymbol{M}(m)}(j, 1)$. Then $C_{i}^{+} \subseteq C_{i}^{*}$. Define

$$
J_{i}^{*} \equiv J_{i}^{*}(m)=\bigcup\left\{C_{j}^{*}(m): j \in \mathcal{N}_{\boldsymbol{M}(m)}(i, K)\right\},
$$




\section{Normal Approximation in Large Network Models}

which contains $J_{i}$. We construct a hybrid branching process

$$
\mathfrak{X}_{r_{n}}^{H}\left(X_{i}, Z_{i} ; K\right)
$$

whose size dominates $\left|J_{i}^{*}\right|$ for sufficiently large $m, n$. First, for any $(x, z) \in \operatorname{supp}\left(X_{i}, Z_{i}\right)$, define a smaller hybrid branching process

$$
\mathfrak{X}_{r_{n}}^{+}(x, z),
$$

constructed by generating $\mathfrak{X}_{r_{n}}^{D}(x, z)$, and then for each particle (in any generation) of type $\left(x^{\prime}, z^{\prime}\right)$ in $\mathfrak{X}_{r_{n}}^{D}(x, z)$, generating a branching process $\mathfrak{X}_{r_{n}}^{M}\left(x^{\prime}, z^{\prime} ; 1\right)$ independently across particles conditional on $\mathfrak{X}_{r_{n}}^{D}(x, z)$. Using Lemma A.2, $\left|\mathfrak{X}_{r_{n}}^{+}(x, z)\right|$ stochastically dominates $\left|C_{i}^{*}\right|$ conditional on $\left(X_{i}, Z_{i}\right)=(x, z)$. Next construct (A.7) by generating the fixed-depth process $\mathfrak{X}_{r_{n}}^{M}\left(X_{i}, Z_{i} ; K\right)$ (whose size dominates that of $\mathcal{N}_{\boldsymbol{M}}(i, K)$ in (A.6)), and then for each particle (in any generation) of type $\left(x^{\prime}, z^{\prime}\right)$ in $\mathfrak{X}_{r_{n}}^{M}\left(X_{i}, Z_{i} ; K\right)$, generating the smaller hybrid process $\mathfrak{X}_{r_{n}}^{+}\left(x^{\prime}, z^{\prime}\right)$ independently across particles conditional on $\mathfrak{X}_{r_{n}}^{M}\left(X_{i}, Z_{i} ; K\right)$. Using Lemma A.2, $\left|\mathfrak{X}_{r_{n}}^{H}\left(X_{i}, Z_{i} ; K\right)\right|$ stochastically dominates $\left|J_{i}^{*}(m)\right|$, and therefore $\left|J_{i}(m)\right|$, as desired. The same result holds if we replace $m$ with $N_{n}+1$.

\section{A.2 Tail Bounds}

Recall the definition of $J_{i}(m)$ from (A.2).

Lemma A.3. Under Assumptions 1, 7, and 9, for any $k \in\{1, \ldots, 4\}$ and $k^{\prime} \in\{1,2\}$, there exist $n_{0}, \epsilon>0$ such that $\lim \sup _{w \rightarrow \infty} w^{-1} \max \left\{\log \tilde{\tau}_{b, \epsilon}(w), \log \tilde{\tau}_{p}(w)\right\}<0$, where

$$
\begin{aligned}
& \tilde{\tau}_{b, \epsilon}(w)=\sup _{n>n_{0}} \sup _{m \in((1-\epsilon) n,(1+\epsilon) n)} \sup _{\boldsymbol{x}_{k} \in \operatorname{supp}(f)^{k}} \mathbf{P}\left(\left|J_{1}(m+k)\right|>w \mid \boldsymbol{X}_{k}=\boldsymbol{x}_{k}\right), \\
& \tilde{\tau}_{p}(w)=\sup _{n>n_{0}} \sup _{\boldsymbol{x}_{k^{\prime}} \in \operatorname{supp}(f)^{k^{\prime}}} \mathbf{P}\left(\left|J_{1}\left(N_{n}+k^{\prime}\right)\right|>w \mid \boldsymbol{X}_{k^{\prime}}=\boldsymbol{x}_{k^{\prime}}\right) .
\end{aligned}
$$

Proof. We prove the result for $\tilde{\tau}_{b, \epsilon}(w), k=3$, and $k^{\prime}=2$. The argument for the other cases is similar. Fix $m \in \mathbb{N}$, and recall the definition of $J_{i}^{*}(m)$ from (A.6). For any $H \subseteq \mathcal{N}_{m}$ with $i \notin H$, let $J_{i}^{*}(m, H) \equiv J_{i}^{*}(m) \backslash H$. We first prove

$$
J_{1}^{*}(m) \subseteq J_{1}^{*}(m,\{2\}) \cup J_{2}^{*}(m,\{1\}) .
$$




\section{LEUNG AND MOON}

Let $\boldsymbol{M}^{-}$and $\boldsymbol{D}^{-}$be the networks obtained from $\boldsymbol{M}$ and $\boldsymbol{D}$ respectively by deleting node 1 and its links. Fix $i \in J_{1}^{*}(m)$. If $i=2$, then clearly $i \in J_{2}^{*}(m,\{1\})$, so suppose $i \neq 2$. Then either (a) $i \in \mathcal{N}_{\boldsymbol{M}}(1, K)$ or (b) $i \in C_{j}^{*}$ for some $j \in \mathcal{N}_{\boldsymbol{M}}(1, K)$, where $C_{j}^{*}$ is defined prior to (A.6). For case (a), if $i \notin J_{1}^{*}(m,\{2\})$, then node 2 must lie on a path of length at most $K$ in $\boldsymbol{M}$ connecting $i$ and node 1 . Therefore $i \in \mathcal{N}_{\boldsymbol{M}^{-}}(2, K)$, so $i \in J_{2}^{*}(m,\{1\})$. For case (b), if $i \notin J_{1}^{*}(m,\{2\})$, then there are two possibilities. The first is that $\boldsymbol{M}_{2 i}=1$, in which case $i \in \mathcal{N}_{\boldsymbol{M}^{-}}(2, K)$. The second is that node 2 lies on some path in $\boldsymbol{D}$ connecting $i$ and some node $j$, in which case nodes 2 and $i$ are connected through a path in $\boldsymbol{D}^{-}$. Thus, in both cases, either $i \in J_{1}^{*}(m,\{2\})$ or $i \in J_{2}^{*}(m,\{1\})$.

Applying the logic of (A.8) several times yields

$$
\begin{aligned}
J_{1}^{*}(m) \subseteq J_{1}^{*}(m & ,\{3\}) \cup J_{3}^{*}(m,\{1\}) \\
& \subseteq\left(J_{1}^{*}(m,\{2,3\}) \cup J_{2}^{*}(m,\{1,3\})\right) \cup\left(J_{3}^{*}(m,\{1,2\}) \cup J_{2}^{*}(m,\{1,3\})\right) .
\end{aligned}
$$

Since $\left|J_{i}(m)\right| \leqslant\left|J_{i}^{*}(m)\right|$, by the previous equation, it suffices to prove the result replacing $J_{1}(m+3)$ and $J_{1}\left(N_{n}+2\right)$ in the definitions of $\tilde{\tau}_{b, \epsilon}(t)$ and $\tilde{\tau}_{p}(t)$ with $J_{3}^{*}(m+$ $3,\{1,2\})$ and $J_{1}^{*}\left(N_{n}+2,\{2\}\right)$, respectively. For sufficiently large $m, n$, the sizes of these sets are both stochastically dominated by $\left|\mathfrak{X}_{r_{n}}^{H}\left(X_{3}, Z_{3} ; K\right)\right|$ defined in (A.7), as shown in $\S$ A.1. By Lemma SA.2.3, $\left|\mathfrak{X}_{r_{n}}^{H}\left(X_{3}, Z_{3} ; K\right)\right|$ satisfies the desired exponential tail bound.

Lemma A.4. Suppose Assumptions 1, 7, and 9 and the conclusion of Lemma A.3 hold. For $n, m \in \mathbb{N}$ sufficiently large, $k \in\{1, \ldots, 4\}$, and $k^{\prime} \in\{1,2\}$, there exist $n_{0}, \epsilon>0$ such that $\lim \sup _{w \rightarrow \infty} w^{-1} \max \left\{\log \tau_{b, \epsilon}^{*}(w), \log \tau_{p}^{*}(w)\right\}<0$, where

$$
\begin{aligned}
& \tau_{b, \epsilon}^{*}(w)=\sup _{n>n_{0}} \sup _{m \in((1-\epsilon) n,(1+\epsilon) n)} \sup _{\boldsymbol{x}_{k} \in \operatorname{supp}(f)^{k}} \mathbf{P}\left(\max _{i \in J_{1}(m+k)} r_{n}^{-1}\left\|X_{1}-X_{i}\right\|>w \mid \boldsymbol{X}_{k}=\boldsymbol{x}_{k}\right), \\
& \tau_{p}^{*}(w)=\sup _{n>n_{0}} \sup _{\boldsymbol{x}_{k^{\prime}} \in \operatorname{supp}(f)^{k^{\prime}}} \mathbf{P}\left(\max _{i \in J_{1}\left(N_{n}+k^{\prime}\right)} r_{n}^{-1}\left\|X_{1}-X_{i}\right\|>w \mid \boldsymbol{X}_{k^{\prime}}=\boldsymbol{x}_{k^{\prime}}\right) .
\end{aligned}
$$

Proof. Since $J_{i}(m) \subseteq J_{i}^{*}(m)$ (A.6), it suffices to prove the result substituting $J_{1}^{*}(m+k)$ and $J_{1}^{*}\left(N_{n}+k^{\prime}\right)$ for $J_{1}(m+k)$ and $J_{1}\left(N_{n}+k^{\prime}\right)$, respectively. We prove the result for $J_{1}^{*}(m+k)$, as the argument is the same for $J_{1}^{*}\left(N_{n}+k^{\prime}\right)$. By the law of 


\section{Normal Approximation in Large Network Models}

total probability,

$$
\begin{aligned}
\mathbf{P}\left(\max _{i \in J_{1}^{*}(m+k)} r_{n}^{-1}\left\|X_{1}-X_{i}\right\|>w \mid \boldsymbol{X}_{k}=\boldsymbol{x}_{k}\right) \leqslant \mathbf{P}\left(\left|J_{1}^{*}(m+k)\right|>w^{\prime} \mid \boldsymbol{X}_{k}=\boldsymbol{x}_{k}\right) \\
+\mathbf{P}\left(\max _{i \in J_{1}^{*}(m+k)} r_{n}^{-1}\left\|X_{1}-X_{i}\right\|>w \cap\left|J_{1}^{*}(m+k)\right| \leqslant w^{\prime} \mid \boldsymbol{X}_{k}=\boldsymbol{x}_{k}\right) .
\end{aligned}
$$

Below we choose $w^{\prime}$ to be a linear, increasing function of $w$, so the first term on the right-hand side obeys the required exponential tail bound in $w$ by Lemma A.3.

Consider the second term. Under the event that $\left|J_{1}^{*}(m+k)\right| \leqslant w^{\prime}$, for $C_{i}^{*}(m+k)$ defined prior to (A.6), we have $C_{i}^{*}(m+k) \subseteq \mathcal{N}_{\boldsymbol{D}(m+k)}\left(i, w^{\prime}\right)$ for all $i \in \mathcal{N}_{\boldsymbol{M}(m+k)}(1, K)$. Defining

$$
\mathcal{N}_{\boldsymbol{J}(m+k)}\left(i, K, w^{\prime}\right)=\bigcup\left\{\mathcal{N}_{\boldsymbol{D}(m+k)}\left(j, w^{\prime}\right): j \in \mathcal{N}_{\boldsymbol{M}(m+k)}(i, K)\right\}
$$

this implies $J_{1}^{*}(m+k) \subseteq \mathcal{N}_{\boldsymbol{J}(m+k)}\left(1, K, w^{\prime}\right)$. Therefore,

$$
\begin{aligned}
\mathbf{P}\left(\max _{i \in J_{1}^{*}(m+k)} r_{n}^{-1}\left\|X_{1}-X_{i}\right\|>w \cap\left|J_{1}^{*}(m+k)\right| \leqslant w^{\prime} \mid \boldsymbol{X}_{k}=\boldsymbol{x}_{k}\right) \\
\quad \leqslant \mathbf{P}\left(\max _{i \in \mathcal{N}_{\boldsymbol{J}(m+k)}\left(1, K, w^{\prime}\right)} r_{n}^{-1}\left\|X_{1}-X_{i}\right\|>w \mid \boldsymbol{X}_{k}=\boldsymbol{x}_{k}\right) .
\end{aligned}
$$

Choose $w^{\prime}=\alpha w$ for some $\alpha>0$, and define the right-hand side of (A.10) as $\delta(w)$. By Lemma SA.2.4, $\delta(w)=O\left(w^{d+1} \exp \{-c w\}\right)$ for some $c>0$, so $\lim \sup _{w \rightarrow \infty} w^{-1} \log \delta(w)<$ 0 , as desired.

\section{B Bounded Subnetwork Moments}

This section formally defines subnetwork counts, introduced in Example 4, and verifies Assumption 6 for these moments. Fix $h \in \mathbb{N} \backslash\{1\}$, and let $a_{h}$ be a connected network on $\{1, \ldots, h\}$ (a network is connected if the path distance between any pair of nodes is finite). For $H \subset \mathcal{N}_{n}$ with $|H|=h$, we say $\boldsymbol{A}_{H}$ is isomorphic to $a_{h}$ if there exists a bijection $\pi: \mathcal{N}_{n} \rightarrow \mathcal{N}_{n}$ such that $\pi(\boldsymbol{A})_{H}=a_{h}$, where $\pi(\boldsymbol{A})$ is the permuted adjacency matrix $\left(A_{\pi(i) \pi(j)}\right)_{i, j=1}^{n}$. If $\boldsymbol{A}_{H}$ is isomorphic to $a_{h}$, we write $\boldsymbol{A}_{H} \cong a_{h}$. 


\section{LEUNG AND MOON}

Let $[n]_{h}$ be the set of subsets of $\mathcal{N}_{n}$ of size $h$. The subnetwork count for $a_{h}$ is

$$
\sum_{H \in[n]_{h}} \mathbf{1}\left\{\boldsymbol{A}_{H} \cong a_{h}\right\}
$$

This is counts "unlabeled" subnetworks isomorphic to $a_{h}$. To rewrite it as a (scaled) network moment $\sum_{i=1}^{n} \psi_{i}\left(\mathcal{N}_{n}\right)$, we observe that this equals the corresponding count of "labeled" subnetworks divided by a constant. Formally,

$$
h ! \sum_{H \in[n]_{h}} \mathbf{1}\left\{\boldsymbol{A}_{H} \cong a_{h}\right\}=\sum_{i_{1} \in \mathcal{N}_{n}} \underbrace{\sum_{i_{2} \in \mathcal{N}_{n} \backslash\left\{i_{1}\right\}} \ldots \sum_{i_{h} \in \mathcal{N}_{n} \backslash\left\{i_{1}, \ldots, i_{h-1}\right\}} \mathbf{1}\left\{\boldsymbol{A}_{\left\{i_{1}, \ldots, i_{h}\right\}} \cong a_{h}\right\}}_{\psi_{i_{1}}\left(\mathcal{N}_{n}\right)} .
$$

The right-hand side counts labeled subnetworks isomorphic to $a_{h}$, which will be our object of analysis in what follows.

Equation (5.3) of Sheng (2020) uses subnetwork counts to define moment inequalities. She scales the counts by $\left(\begin{array}{l}n \\ h\end{array}\right)^{-1}$ since she considers a setting with many independent networks. In our sparse, single large network setting, the correct scaling is instead $n^{-1}$, resulting in the network moment $n^{-1} \sum_{i=1}^{n} \psi_{i}\left(\mathcal{N}_{n}\right)$ for $\psi_{i}\left(\mathcal{N}_{n}\right)$ defined in (B.1).

Proposition B.1. Under Assumption 1, Assumption 6 holds for node statistics $\psi_{i}\left(\mathcal{N}_{n}\right)$ of the form given in (B.1).

Proof. Per the setup of Assumption 6, let the number of nodes be $m+k$, where either $m$ is a nonrandom element of $\mathbb{N}$ or $m=N_{n}$. We focus on the case $k=1$ since $k=2$ is similar. In the case where $m=N_{n}$, the setup of Assumption 6 also considers models where the set of nodes is a subset $H_{n} \cup\{1\}$ of $\mathcal{N}_{m+k}$, but since this only reduces the subnetwork count, it is sufficient to consider a model with all nodes. Define

$$
\psi_{1}\left(\mathcal{N}_{m+k}\right)=\sum_{i_{1} \in \mathcal{N}_{m+k} \backslash\{1\}} \ldots \sum_{i_{h-1} \in \mathcal{N}_{m+k} \backslash\left\{1, i_{1}, \ldots, i_{h-2}\right\}} \mathbf{1}\left\{\boldsymbol{A}_{\left\{1, i_{1}, \ldots, i_{h-1}\right\}} \cong a_{h}\right\}
$$

Since $a_{h}$ is a connected network on $\{1, \ldots, h\}$, any node in the network is at most path distance $h-1$ from node 1 . Therefore, without loss of generality, we may restrict the summations in (B.2) to each be over elements of $\mathcal{N}_{\boldsymbol{A}}(1, h-1)$. Then the right-hand 
side of (B.2) is bounded by $\left|\mathcal{N}_{\boldsymbol{A}}(1, h-1)\right|^{h-1}$.

Recall the definition of the network $\boldsymbol{M}$ from (A.1). Since $A_{i j} \leqslant M_{i j}, \boldsymbol{A}$ is a subnetwork of $\boldsymbol{M}$, so

$$
\left|\mathcal{N}_{\boldsymbol{A}}(1, h-1)\right|^{h-1} \leqslant\left|\mathcal{N}_{\boldsymbol{M}}(1, h-1)\right|^{h-1} .
$$

By Lemma A.2, $\left|\mathcal{N}_{\boldsymbol{M}}(1, h-1)\right|$ is stochastically dominated by the size of a branching process $\left|\mathfrak{X}_{r_{n}}^{M}(x, z ; h-1)\right|$ conditional on $\left(X_{1}, Z_{1}\right)=(x, z)$. By Lemma SA.2.2, the distribution of $\left|\mathfrak{X}_{r}^{M}(x, z ; h-1)\right|$ has exponential tails uniformly in $x, z, r$. It follows that $\left|\mathcal{N}_{\boldsymbol{M}}(1, h-1)\right|^{h-1}$ has uniformly bounded $p$ moments for any $p>2$.

\section{References}

Badev, A., "Discrete Games in Endogenous Networks: Theory and Policy," FRB working paper, 2013.

Barabási, A., Network Science, Cambridge University Press, 2015.

Barabási, A. and R. Albert, "Emergence of Scaling in Random Networks," Science, 1999, 286 (5439), 509-512.

Bickel, P., A. Chen, and E. Levina, "The Method of Moments and Degree Distributions for Network Models," Annals of Statistics, 2011, 39 (5), 2280-2301.

Bollobás, B. and O. Riordan, "A Simple Branching Process Approach to the Phase Transition in $G(n, p)$," Electronic Journal of Combinatorics, 2012, 19 (4), P21.

Bollobás, B., S. Janson, and O. Riordan, "The Phase Transition in Inhomogeneous Random Graphs," Random Structures and Algorithms, 2007, 31 (1), 3-122.

Boucher, V. and I. Mourifié, "My Friend Far Far Away: A Random Field Approach to Exponential Random Graph Models," Econometrics Journal, 2017, 20 (3), S14-S46.

Bramoullé, Y., H. Djebbari, and B. Fortin, "Identification of Peer Effects through Social Networks," Journal of Econometrics, 2009, 150 (1), 41-55. 


\section{LEUNG AND MOON}

Breza, E., A. Chandrasekhar, T. McCormick, and M. Pan, "Using Aggregated Relational Data to Feasibly Identify Network Structure without Network Data," American Economic Review, 2020, 110 (8), 2454-2484.

Canay, I., J. Romano, and A. Shaikh, "Randomization Tests Under an Approximate Symmetry Assumption," Econometrica, 2017, 85 (3), 1013-1030.

Carrell, S., B. Sacerdote, and J. West, "From Natural Variation to Optimal Policy? The Importance of Endogenous Peer Group Formation," Econometrica, 2013, $81(3), 855-882$.

Chandrasekhar, A., "Econometrics of Network Formation," in Y. Bramoullé, A. Galeotti, and B. Rogers, eds., Oxford Handbook on the Econometrics of Networks, 2016.

_ and M. Jackson, "A Network Formation Model Based on Subgraphs," Stanford working paper, 2021.

Charbonneau, K., "Multiple Fixed Effects in Binary Response Panel Data Models," The Econometrics Journal, 2017, 20 (3), S1-S13.

Chen, L. and Q. Shao, "Normal Approximation Under Local Dependence," Annals of Probability, 2004, 32 (3), 1985-2028.

Christakis, N., J. Fowler, G. Imbens, and K. Kalyanaraman, "An Empirical Model for Strategic Network Formation," in "The Econometric Analysis of Network Data," Elsevier, 2020, pp. 123-148.

de Jong, Robert M. and Tiemen Woutersen, "Dynamic Time Series Binary Choice," Econometric Theory, 2011, 27, 673-702.

Durlauf, S., "Associational Redistribution: A Defense," Politics 6 Society, 1996, 24 (4), 391-410.

Dzemski, A., "An Empirical Model of Dyadic Link Formation in a Network with Unobserved Heterogeneity," Review of Economics and Statistics, 2019, 101 (5), $763-776$.

Fafchamps, M. and F. Gubert, "The Formation of Risk-Sharing Networks," Journal of Economic Development, 2007, 83, 326-350. 
Normal Approximation in Large Network Models

Goldsmith-Pinkham, P. and G. Imbens, "Social Networks and the Identification of Peer Effects," Journal of Business and Economic Statistics, 2013, 31 (3), 253264.

Graham, B., "An Econometric Model of Network Formation with Degree Heterogeneity," Econometrica, 2017, 85 (1), 1033-1063.

Hoff, P., A. Raftery, and M. Handcock, "Latent Space Approaches to Social Network Analysis," Journal of the American Statistical Association, 2002, 97 (460), 1090-1098.

Jackson, M., Social and Economic Networks, Princeton University Press, 2010.

_ , B. Rogers, and Y. Zenou, "Networks: An Economic Perspective," in "Oxford Handbook of Social Network Analysis" 2021, pp. 535-562.

Jochmans, K., "Semiparametric Analysis of Network Formation," Journal of Business and Economic Statistics, 2018, 36 (4), 705-713.

Klenke, A., Probability Theory: A Comprehensive Course, Springer Science \& Business Media, 2013.

Kuersteiner, G., "Limit Theorems for Data with Network Structure," arXiv preprint arXiv:1908.02375, 2019.

Last, G. and M. Penrose, Lectures on the Poisson Process, Vol. 7, Cambridge University Press, 2017.

Leung, M., "A Random-Field Approach to Inference in Large Models of Network Formation," Stanford working paper, 2015.

_ , "Two-Step Estimation of Network-Formation Models with Incomplete Information," Journal of Econometrics, 2015, 188 (1), 182-195.

_ , "Inference in Models of Discrete Choice with Social Interactions Using Network Data," arXiv preprint arXiv:1911.07106, 2019.

_ , "A Weak Law for Moments of Pairwise-Stable Networks," Journal of Econometrics, 2019, 210 (2), 310-326. 


\section{LEUNG AND MOON}

_ , "Equilibrium Computation in Discrete Network Games," Quantitative Economics, 2020, 11 (4), 1325-1347.

_ , "Dependence-Robust Inference Using Resampled Statistics," Journal of Applied Econometrics, 2022, 37 (2), 270-285.

— and R. Moon, "Normal Approximation in Large Network Models," arXiv preprint arXiv:1904.11060v1, 2019.

McPherson, M., L. Smith-Lovin, and J. Cook, "Birds of a Feather: Homophily in Social Networks," Annual Review of Sociology, 2001, 27, 415-444.

Mele, A., "A Structural Model of Dense Network Formation," Econometrica, 2017, $85(3), 825-850$.

_ , "Does School Desegregation Promote Diverse Interactions? An Equilibrium Model of Segregation Within Schools," American Economic Journal: Economic Policy, 2020, $12(2), 228-57$.

_ , "A Structural Model of Homophily and Clustering in Social Networks," Journal of Business and Economic Statistics, 2022, 40 (3), 1377-1389.

Menzel, K., "Strategic Network Formation with Many Agents," NYU working paper, 2017.

Penrose, M., Random Geometric Graphs, Oxford University Press, 2003.

_ , "Gaussian Limits for Random Geometric Measures," Electronic Journal of Probability, 2007, 12, 989-1035.

_ and J. Yukich, "Weak Laws of Large Numbers in Geometric Probability," Annals of Applied Probability, 2003, pp. 277-303.

_ and _, "Normal Approximation in Geometric Probability," Stein's Method and Applications, Lecture Note Series, Institute for Mathematical Sciences, National University of Singapore, 2005, 5, 37-58.

Powell, W., D. White, K. Koput, and J. Owen-Smith, "Network Dynamics and Field Evolution: The Growth of Interorganizational Collaboration in the Life Sciences," American Journal of Sociology, 2005, 110 (4), 1132-1205. 


\section{Normal Approximation in Large Network Models}

Ridder, G. and S. Sheng, "Two-Step Estimation of a Strategic Network Formation Model with Clustering," arXiv preprint arXiv:2001.03838, 2022.

Schneider, R. and W. Weil, Stochastic and Integral Geometry, Springer Science \& Business Media, 2008.

Sheng, S., "A Structural Econometric Analysis of Network Formation Games Through Subnetworks," Econometrica, 2020, 88 (5), 1829-1858. 


\section{Supplementary Appendix}

\section{SA.1 Proof of Theorem 1}

Proof of TheOrem 1. Recall that $d_{\psi}$ is the dimension of the range of $\psi(\cdot)$. For the case $d_{\psi}=1$, the result follows directly from Theorem SA.1.2. For the case $d_{\psi}>1$, let $t \in \mathbb{R}^{d_{\psi}} \backslash\{\mathbf{0}\}$. The 1 -dimensional statistic $\psi_{i}^{*}\left(\mathcal{N}_{n}\right) \equiv t^{\prime} \psi_{i}\left(\mathcal{N}_{n}\right)$ satisfies Assumptions 5 and 6 since $\psi(\cdot)$ satisfies them, so the result follows from the Cramér-Wold device.

\section{SA.1.1 Poissonization}

This subsection proves a CLT for the Poissonized model in which the set of nodes is $\mathcal{N}_{N_{n}}$ rather than $\mathcal{N}_{n}$, where $N_{n}$ is defined in (11). Throughout, we assume $d_{\psi}=1$. Define $\tilde{\sigma}_{n}^{2}=n^{-1} \operatorname{Var}\left(\sum_{i=1}^{N_{n}} \psi_{i}\left(\mathcal{N}_{N_{n}}\right)\right)$.

Theorem SA.1.1. Suppose $d_{\psi}=1$. Under Assumptions 5 and $6, \sup _{n} \tilde{\sigma}_{n}^{2}<\infty$, and if additionally $\liminf \operatorname{in}_{n \rightarrow \infty} \tilde{\sigma}_{n}^{2}>0$, then

$$
\tilde{\sigma}_{n}^{-1} \frac{1}{\sqrt{n}}\left(\sum_{i=1}^{N_{n}} \psi_{i}\left(\mathcal{N}_{N_{n}}\right)-\mathbf{E}\left[\sum_{i=1}^{N_{n}} \psi_{i}\left(\mathcal{N}_{N_{n}}\right)\right]\right) \stackrel{d}{\longrightarrow} \mathcal{N}(0,1)
$$

The proof follows that of Theorem 2.1 of Penrose and Yukich (2005) (henceforth PY). For completeness, we next restate its key elements using our notation.

Remark SA.1.1 (Related Literature). That PY's proof essentially carries over to our setting is perhaps not obvious due to several differences between our settings. First, our definition of exponential stabilization differs since, as discussed in $\S 3.3$, we only require invariance of node statistics to the removal of nodes outside the radius of stabilization, whereas PY demand invariance to removal and addition. Second, PY's model has no random-utility shocks $\zeta_{i j}$, but adding these to the model turns out to have no effect on the argument due to their high degree of independence; they are 


\section{Normal Approximation in Large Network Models}

independent across pairs, whereas types are dependent across pairs with a common node. Third, they define $\boldsymbol{T}_{N_{n}}$ as a marked Poisson point process, which is equivalent to our representation because (a) our set of positions $\boldsymbol{X}_{N_{n}}=\left(X_{i}\right)_{i=1}^{N_{n}}$ has the same distribution as $\mathcal{P}_{n f}$, the Poisson point process on $\mathbb{R}^{d}$ with intensity function $n f(\cdot)$ (Penrose, 2003, Proposition 1.5), and (b) the "marks" $Z_{i}$ associated with each $X_{i}$ are independent across nodes, each only potentially correlated with its own position $X_{i}{ }^{7}$ Fourth, PY consider the simpler case where $X_{i} \Perp Z_{i}$ for each $i$ since this is sufficient for their applications. We allow for dependence, but this turns out not to affect the argument. Finally, PY consider moments of the form

$$
\frac{1}{n} \sum_{i=1}^{N_{n}} \xi\left(\left(X_{i}, Z_{i}\right), r_{n}^{-1} \boldsymbol{T}_{N_{n}}\right)
$$

This differs from $\psi(\cdot)$ because $\boldsymbol{A}$ is not an argument (nor is $\boldsymbol{\zeta}_{N_{n}}$ for reasons previously discussed). However, since $\boldsymbol{A}=\lambda_{N_{n}}\left(r_{n}^{-1} \boldsymbol{T}_{N_{n}}, \boldsymbol{\zeta}_{N_{n}}\right)$ by Assumption 3, we can define

$$
\xi\left(i, r_{n}^{-1} \boldsymbol{T}_{N_{n}}, \boldsymbol{\zeta}_{N_{n}}\right) \equiv \psi\left(i, r_{n}^{-1} \boldsymbol{T}_{N_{n}}, \boldsymbol{\zeta}_{N_{n}}, \lambda_{N_{n}}\left(r_{n}^{-1} \boldsymbol{T}_{N_{n}}, \boldsymbol{\zeta}_{N_{n}}\right)\right),
$$

in which case this coincides with PY's setup except for the presence of $\boldsymbol{\zeta}_{N_{n}}$.

The basis of the proof is a dependency graph CLT due to Chen and Shao (2004). The technique is to approximate $\sum_{i=1}^{N_{n}} \psi_{i}\left(\mathcal{N}_{N_{n}}\right)$ by a sum $\sum_{i=1}^{n} W_{i}$, where the dependence structure of the summands can be characterized in terms of a network ("dependency graph") $\boldsymbol{G}$ in the sense that observations unlinked in $\boldsymbol{G}$ are independent.

Definition 2. A network $\boldsymbol{G}$ over $n$ nodes (with self-links) is a dependency graph for data $\left\{W_{i}\right\}_{i=1}^{n}$ if for any $S_{1}, S_{2} \subseteq \mathcal{N}_{n}$ such that $G_{i j}=0$ for all $i \in S_{1}$ and $j \in S_{2}$, we have $\left\{W_{i}: i \in S_{1}\right\} \Perp\left\{W_{j}: j \in S_{2}\right\}$.

Let $\|W\|_{p}$ be the $L_{p^{-}}$-norm of $W$ and $\Phi(\cdot)$ the standard normal CDF.

Lemma SA.1.1 (Dependency Graph CLT). Let $q \in(2,3)$ and $W=\sum_{i=1}^{n} W_{i}$. Suppose $\boldsymbol{G}$ is a dependency graph for $\left\{W_{i}\right\}_{i=1}^{n}$, and let $\Gamma=\max _{i} \sum_{j \neq i} G_{i j}$. Further suppose

\footnotetext{
${ }^{7}$ See e.g. Last and Penrose (2017) for the definition of a marked Poisson point process.
} 


\section{LEUNG AND MOON}

$\mathbf{E}\left[W^{2}\right]=1, \mathbf{E}\left[W_{i}\right]=0$, and $\left\|W_{i}\right\|_{q} \leqslant \theta$ for all $i$ and some $\theta>0$. Then

$$
\sup _{t}|\mathbf{P}(W \leqslant t)-\Phi(t)| \leqslant 75 \theta^{q} \Gamma^{5(q-1)} n .
$$

Proof. This is Theorem 2.7 of Chen and Shao (2004).

We next introduce some notation. Let $\rho_{n}=\alpha \log n$ for some $\alpha$ sufficiently large that, for some $C>0$, all $n \geqslant 1$, and $\tau_{p}(\cdot)$ in Assumption 5,

$$
\rho_{n}^{d / p}\left(n \tau_{p}\left(\rho_{n}\right)\right)^{(q-2) /(2 q)}<C n^{-4}, \quad \tau_{p}\left(\rho_{n}\right)<C n^{-3}, \quad \rho_{n}^{d}<C n^{p /(p+2)}
$$

where $p$ is given in Assumption 6 and $d$ is the dimension of $X_{1}$.

As discussed above, $\boldsymbol{X}_{N_{n}}$ has the same distribution as the Poisson point process $\mathcal{P}_{n f}$. It can then be represented as a different collection of independent random variables that is more useful for the argument that follows. Let $s_{n}=r_{n} \rho_{n}$ and $V_{n}$ be the number of cubes of the form $Q=\prod_{i=1}^{d}\left[j_{i} s_{n},\left(j_{i}+1\right) s_{n}\right)$, where $j_{i} \in \mathbb{Z}$ for all $i$, such that $Q$ has positive density under $f$. Label these cubes $Q_{1}, \ldots, Q_{V_{n}}$. Fix any $1 \leqslant i \leqslant V_{n}$. Note that $\left|\boldsymbol{X}_{N_{n}} \cap Q_{i}\right|$ has the same distribution as $N_{i} \sim \operatorname{Poisson}\left(\nu_{i}\right)$ for $\nu_{i}=n \int_{Q_{i}} f(x) \mathrm{d} x$. Label the points of $\boldsymbol{X}_{N_{n}} \cap Q_{i}$ as $X_{i 1}, \ldots, X_{i N_{i}}$, which, conditional on $N_{i}$, are i.i.d. draws from a density $f(\cdot) / \int_{Q_{i}} f(x) \mathrm{d} x$. Under this representation,

$$
\boldsymbol{X}_{N_{n}}=\cup_{i=1}^{V_{n}}\left\{X_{i j}\right\}_{j=1}^{N_{i}}
$$

In what follows, we will often use this double-indexed labeling for nodes under this representation, where we label a node $k$ by $i j$ if its position $X_{k}$ corresponds to $X_{i j}$. We denote node $i j$ 's node statistic by

$$
\psi_{i j}\left(\mathcal{N}_{N_{n}}\right)
$$

The next lemma is mostly a restatement of Lemma 4.3 of Penrose and Yukich (2005), translated to our notation. ${ }^{8}$ The main difference relative to PY is the presence of random-utility shocks $\boldsymbol{\zeta}_{N_{n}}$. Since the proof only involves moment calculations that exploit the Poisson distribution of $N_{i}$, all of the expressions integrate over $\boldsymbol{\zeta}_{N_{n}}$.

\footnotetext{
${ }^{8}$ The translation dictionary is as follows: replace $\lambda$ (their notation) with $n$ (our notation), $\lambda^{-1 / d}$ with $r_{n}, \kappa$ with $f, A_{\lambda}$ with $\operatorname{supp}(f), f$ with the function mapping any real number to $1, \mathcal{P}_{\lambda}$ with $\boldsymbol{X}_{N_{n}}$, and $\xi_{\lambda}\left(X_{i, j}, \mathcal{P}_{\lambda}\right)$ with $\psi_{i j}\left(\mathcal{N}_{N_{n}}\right)$ (we define the $i j$ notation below).
} 


\section{Normal Approximation in Large Network Models}

Because the shocks are independent of $\boldsymbol{X}_{N_{n}}$, their presence does not materially affect the proof.

Lemma SA.1.2. For any $S \subseteq \mathbb{R}^{d}$, let $\mathcal{N}_{N_{n}}^{\prime}(S)=\left\{i \in \mathcal{N}_{N_{n}}: X_{i} \in S\right\}$. Under Assumption 6, for $p$ given in the assumption and $1<q<p$, there exists $C>0$ such that $\left\|\sum_{j=1}^{\infty}\left|\psi_{i j}\left(\mathcal{N}_{N_{n}}^{\prime}(S)\right) \mathbf{1}\left\{j \leqslant N_{i}\right\}\right|\right\|_{q} \leqslant C \rho_{n}^{d(p+1) / p}$ for all $1 \leqslant i \leqslant V_{n}$ and $S \subseteq \mathbb{R}^{d}$.

Since the lemma is a fairly straightforward consequence of Assumption 6, we omit the proof.

Proof of TheOrem SA.1.1. We mostly follow PY's proof of Theorem 2.1 with some minor modifications, reorganization, and elaboration. That $\sup _{n} \tilde{\sigma}_{n}^{2}<\infty$ is a consequene of Lemma SA.1.7 below. Abbreviate

$$
\Lambda_{n}=\sum_{i=1}^{N_{n}} \psi_{i}\left(\mathcal{N}_{N_{n}}\right)
$$

We prove the following stronger result: for $q \in(2,3]$ with $q<p$, there exists $C>0$ such that for all $n$ sufficiently large,

$$
\sup _{t}\left|\mathbf{P}\left(\operatorname{Var}\left(\Lambda_{n}\right)^{-1 / 2}\left(\Lambda_{n}-\mathbf{E}\left[\Lambda_{n}\right]\right) \leqslant t\right)-\Phi(t)\right| \leqslant C(\log n)^{d q} n \operatorname{Var}\left(\Lambda_{n}\right)^{-q / 2} .
$$

Since $\operatorname{Var}\left(\Lambda_{n}\right)^{-1}=O\left(n^{-1}\right)$ by assumption, the right-hand side of (SA.1.3) tends to zero, which proves the theorem.

Setup. Under representation (SA.1.2),

$$
\Lambda_{n}=\sum_{i=1}^{V_{n}} \sum_{j=1}^{N_{i}} \psi_{i j}\left(\mathcal{N}_{N_{n}}\right)
$$

The key idea is to approximate this with a similar sum to which the dependency graph CLT (Lemma SA.1.1) more usefully applies.

Recall $\boldsymbol{R}_{i}\left(n, r_{n}\right)$ from Definition 1. Since our setup now indexes nodes by $i j$ for $1 \leqslant i \leqslant V_{n}$ and $1 \leqslant j \leqslant N_{i}$, we instead write $\boldsymbol{R}_{i j}\left(n, r_{n}\right)$. Let $\mathcal{E}_{i j}$ be the event $\left\{\boldsymbol{R}_{i j}\left(N_{n}, r_{n}\right) \leqslant \rho_{n}\right\}$ for $\rho_{n}$ defined prior to (SA.1.1). For any $1 \leqslant i \leqslant V_{n}$, let $Q_{i}^{+}=$ 


\section{LEUNG AND MOON}

$\left\{x \in \mathbb{R}^{d}:\|x-y\| \leqslant r_{n} \rho_{n} \forall y \in Q_{i}\right\}$, the $r_{n} \rho_{n}$-neighborhood of $Q_{i}$. Define

$$
\Lambda_{n}^{\prime}=\sum_{i=1}^{V_{n}} \sum_{j=1}^{N_{i}} \psi_{i j}\left(\mathcal{N}_{N_{n}}^{\prime}\left(Q_{i}^{+}\right)\right) \quad \text { and } \quad \Psi_{i}=\operatorname{Var}\left(\Lambda_{n}^{\prime}\right)^{-1 / 2} \sum_{j=1}^{N_{i}} \psi_{i j}\left(\mathcal{N}_{N_{n}}^{\prime}\left(Q_{i}^{+}\right)\right)
$$

so that $\operatorname{Var}\left(\Lambda_{n}^{\prime}\right)^{-1 / 2} \Lambda_{n}^{\prime}=\sum_{i=1}^{V_{n}} \Psi_{i}{ }^{9}$

We derive a bound on the discrepancy between $\Lambda_{n}$ and $\Lambda_{n}^{\prime}$. By Lemma SA.1.2 and Minkowski's inequality,

$$
\max \left\{\left\|\Lambda_{n}\right\|_{q},\left\|\Lambda_{n}^{\prime}\right\|_{q}\right\} \leqslant C V_{n} \rho_{n}^{d(p+1) / p} \leqslant C n \rho_{n}^{d / p}
$$

By Definition 1, under the event $\mathcal{E}_{n}=\bigcap_{i=1}^{V_{n}} \bigcap_{j=1}^{\infty} \mathcal{E}_{i j}$, we have $\Lambda_{n}^{\prime}=\Lambda_{n}$, so

$$
\left\|\Lambda_{n}-\Lambda_{n}^{\prime}\right\|_{2} \leqslant\left\|\Lambda_{n}-\Lambda_{n}^{\prime}\right\|_{q} \mathbf{P}\left(\mathcal{E}_{n}^{c}\right)^{0.5-1 / q} \leqslant\left(\left\|\Lambda_{n}\right\|_{q}-\left\|\Lambda_{n}^{\prime}\right\|_{q}\right) \mathbf{P}\left(\mathcal{E}_{n}^{c}\right)^{(q-2) /(2 q)}
$$

by the Hölder and Minkowski inequalities. By the law of iterated expectations,

$$
\mathbf{P}\left(\mathcal{E}_{n}^{c}\right) \leqslant \mathbf{E}\left[\sum_{i=1}^{V_{n}} \sum_{j=1}^{N_{i}} \mathbf{1}\left\{\mathcal{E}_{i j}^{c}\right\}\right]=\mathbf{E}\left[\sum_{i=1}^{V_{n}} \sum_{j=1}^{N_{i}} \mathbf{E}\left[\mathbf{1}\left\{\mathcal{E}_{i j}^{c}\right\} \mid \boldsymbol{X}_{N_{n}}, N_{n}\right]\right]
$$

As in the setting of Penrose and Yukich (2005), this is an expectation of pointwise sums (i.e. over the points of $\boldsymbol{X}_{N_{n}}$ ) of functionals of a Poisson point process $\boldsymbol{X}_{N_{n}}$. We may then apply Theorem 1.6 of Penrose (2003) to obtain

$\mathbf{P}\left(\mathcal{E}_{n}^{c}\right) \leqslant \mathbf{E}\left[\sum_{i=1}^{V_{n}} \sum_{j=1}^{N_{i}} \mathbf{1}\left\{\mathcal{E}_{i j}^{c}\right\}\right]=n \int_{\mathbb{R}^{d}} \mathbf{P}\left(\boldsymbol{R}_{i j}\left(N_{n}+1, r_{n}\right)>\rho_{n} \mid X_{i j}=x\right) f(x) \mathrm{d} x \leqslant n \tau_{p}\left(\rho_{n}\right)$,

for $n$ sufficiently large. By (SA.1.4), (SA.1.5), (SA.1.6), our choice of $\rho_{n}$ (SA.1.1), and Assumption 5,

$$
\left\|\Lambda_{n}-\Lambda_{n}^{\prime}\right\|_{2} \leqslant C n^{-3}
$$

Dependency Graph. We construct a dependency graph $\boldsymbol{G}$ for $\left\{\Psi_{i}\right\}_{i=1}^{V_{n}}$ by setting $G_{i j}=1$ inf $\left.\left\{\|x-y\|: x \in Q_{i}, y \in Q_{j}\right\} \leqslant 2 r_{n} \rho_{n}\right\}$. To see that this is a dependency graph, observe that the value of $\Psi_{i}$ is determined solely by (the types of and randomutility shocks between) the subset of nodes with positions in $Q_{i}^{+}$. By Lemma SA.1.3

\footnotetext{
${ }^{9}$ The definition of $\Lambda_{n}^{\prime}$ differs from that of PY's $T_{\lambda}^{\prime}$ to account for the fact our notion of stabilization only demands invariance with respect to the removal, and not the addition, of nodes.
} 


\section{Normal Approximation in Large Network Models}

below, for any two disjoint sets $S_{1}, S_{2} \subseteq \mathbb{R}^{d}$,

$$
\left\{\left(X_{i}, X_{j}, Z_{i}, Z_{j}, \zeta_{i j}\right): i, j \in \mathcal{N}_{N_{n}}^{\prime}\left(S_{1}\right)\right\} \Perp\left\{\left(X_{i}, X_{j}, Z_{i}, Z_{j}, \zeta_{i j}\right): i, j \in \mathcal{N}_{N_{n}}^{\prime}\left(S_{2}\right)\right\} .
$$

Therefore, we have $\left\{\Psi_{k}: k \in Q_{i}\right\} \Perp\left\{\Psi_{\ell}: \ell \in Q_{j}\right\}$ whenever $\inf \left\{\|x-y\|: x \in Q_{i}, y \in\right.$ $\left.Q_{j}\right\}>2 r_{n} \rho_{n}$. Hence, $\boldsymbol{G}$ is a dependency graph.

Note that (SA.1.3) holds trivially when $\operatorname{Var}\left(\Lambda_{n}\right)<1$ for $n$ sufficiently large, so we henceforth consider the case where it is at least 1 . We apply Lemma SA.1.1 to $\left\{W_{i}\right\}_{i=1}^{V_{n}}$ for $W_{i}=\Psi_{i}-\mathbf{E}\left[\Psi_{i}\right]$. To determine a good choice of $\theta$ for applying Lemma SA.1.1, notice by Lemma SA.1.2 that

$$
\left\|\Psi_{i}\right\|_{q} \leqslant C \operatorname{Var}\left(\Lambda_{n}^{\prime}\right)^{-1 / 2}\left\|\sum_{j=1}^{\infty}\left|\psi_{i j}\left(\mathcal{N}_{N_{n}}^{\prime}\left(Q_{i}^{+}\right)\right) \mathbf{1}\left\{j \leqslant N_{i}\right\}\right|\right\|_{q} \leqslant C \operatorname{Var}\left(\Lambda_{n}^{\prime}\right)^{-1 / 2} \rho_{n}^{d(p+1) / p} .
$$

Therefore, we set

$$
\theta=C \operatorname{Var}\left(\Lambda_{n}^{\prime}\right)^{-1 / 2} \rho_{n}^{d(p+1) / p} .
$$

Let $W=\sum_{i=1}^{V_{n}}\left(\Psi_{i}-\mathbf{E}\left[\Psi_{i}\right]\right)$. Since $f$ has bounded support,

$$
V_{n}=O\left(n \rho_{n}^{-d}\right)
$$

Also, there are at most $5^{d}$ other cubes at most $2 r_{n} \rho_{n}$ from cube $Q_{i}$, so $\Gamma \leqslant 5^{d}$. By Lemma SA.1.1,

$$
\sup _{t}|\mathbf{P}(W \leqslant t)-\Phi(t)| \leqslant C n \rho_{n}^{-d} \operatorname{Var}\left(\Lambda_{n}^{\prime}\right)^{-q / 2} \rho_{n}^{d q(p+1) / p}
$$

Obtaining Bound (SA.1.3). We next show that we can replace the right-hand side of (SA.1.10) with the bound on the right-hand side of (SA.1.3). First, we bound the discrepancy between $\operatorname{Var}\left(\Lambda_{n}^{\prime}\right)$ and $\operatorname{Var}\left(\Lambda_{n}\right)$. Trivially,

$$
\operatorname{Var}\left(\Lambda_{n}\right)=\operatorname{Var}\left(\Lambda_{n}^{\prime}\right)+\operatorname{Var}\left(\Lambda_{n}-\Lambda_{n}^{\prime}\right)+2 \operatorname{Cov}\left(\Lambda_{n}^{\prime}, \Lambda_{n}-\Lambda_{n}^{\prime}\right)
$$

We can bound the first term on the right-hand side as follows. By construction, $\Lambda_{n}^{\prime}$ is the sum of $V_{n}$ random variables that have second moments bounded by a constant times $\rho_{n}^{2 d(p+1) / p}$ due to Lemma SA.1.2 for case $q=2$. Also, as previously established, the covariance between any pair of these variables is zero when their indices $i, j$ cor- 


\section{Leung And MoOn}

respond to non-adjacent cubes $Q_{i}, Q_{j}$. For adjacent cubes, the covariance is bounded by a constant times $\rho_{n}^{2 d(p+1) / p}$ by Cauchy-Schwarz. Consequently, by (SA.1.9),

$$
\operatorname{Var}\left(\Lambda_{n}^{\prime}\right)=O\left(n \rho_{n}^{d(p+2) / p}\right)
$$

Combining (SA.1.7), (SA.1.11), and (SA.1.12), by our choice of $\rho_{n}$ (SA.1.1) and Cauchy-Schwarz,

$$
\left|\operatorname{Var}\left(\Lambda_{n}\right)-\operatorname{Var}\left(\Lambda_{n}^{\prime}\right)\right| \leqslant C n^{-2} .
$$

Given $\operatorname{Var}\left(\Lambda_{n}\right) \geqslant 1$ from above, this implies that, for $n$ sufficiently large, $\operatorname{Var}\left(\Lambda_{n}^{\prime}\right) \geqslant$ $\operatorname{Var}\left(\Lambda_{n}\right) / 2$. Hence,

$$
(\mathrm{SA} .1 .10) \leqslant C n \operatorname{Var}\left(\Lambda_{n}\right)^{-q / 2} \rho_{n}^{d q},
$$

which delivers the bound on the right-hand side of (SA.1.3), given $\rho_{n}=\alpha \log n$.

Proving (SA.1.3). However, (SA.1.10) and (SA.1.14) do not immediately deliver (SA.1.3) because they apply to $W=\operatorname{Var}\left(\Lambda_{n}^{\prime}\right)^{-1 / 2}\left(\Lambda_{n}^{\prime}-\mathbf{E}\left[\Lambda_{n}^{\prime}\right]\right)$ rather than the target $\operatorname{Var}\left(\Lambda_{n}\right)^{-1 / 2}\left(\Lambda_{n}-\mathbf{E}\left[\Lambda_{n}\right]\right)$. Let $\tilde{W}=\operatorname{Var}\left(\Lambda_{n}^{\prime}\right)^{-1 / 2}\left(\Lambda_{n}-\mathbf{E}\left[\Lambda_{n}\right]\right)$. Using the Lipschitz property of $\Phi(\cdot)$ and (SA.1.14), elementary calculations yield

$$
\sup _{t}|\mathbf{P}(\tilde{W} \leqslant t)-\Phi(t)| \leqslant C \beta+C n \operatorname{Var}\left(\Lambda_{n}\right)^{-q / 2} \rho_{n}^{d q}+\mathbf{P}(|\tilde{W}-W| \geqslant \beta)
$$

for any $\beta>0$ (Penrose and Yukich, 2005, eq. (4.19)). Also,

$$
|\tilde{W}-W| \leqslant \operatorname{Var}\left(\Lambda_{n}^{\prime}\right)^{-1 / 2}\left(\left|\Lambda_{n}-\Lambda_{n}^{\prime}\right|+\mathbf{E}\left[\left|\Lambda_{n}-\Lambda_{n}^{\prime}\right|\right]\right)
$$

Note that (SA.1.7) implies $\mathbf{E}\left[\left|\Lambda_{n}-\Lambda_{n}^{\prime}\right|\right] \leqslant C n^{-3}$, so under $\mathcal{E}_{n}$, (SA.1.16) $\leqslant C n^{-3}$. On the other hand, $\mathbf{P}\left(\mathcal{E}_{n}^{c}\right) \leqslant C n^{-2}$ by (SA.1.6) and (SA.1.1). So choosing $\beta=C n^{-3}$ in (SA.1.15), we obtain

$$
\sup _{t}|\mathbf{P}(\tilde{W} \leqslant t)-\Phi(t)| \leqslant C n \operatorname{Var}\left(\Lambda_{n}\right)^{-q / 2} \rho_{n}^{d q}+C n^{-2}
$$




\section{Normal Approximation in Large Network Models}

Finally, we turn to bounding the left-hand side of (SA.1.3). We have

$$
\begin{array}{r}
\sup _{t}\left|\mathbf{P}\left(\operatorname{Var}\left(\Lambda_{n}\right)^{-1 / 2}\left(\Lambda_{n}-\mathbf{E}\left[\Lambda_{n}\right]\right) \leqslant t\right)-\Phi(t)\right| \\
\leqslant \sup _{t}\left|\mathbf{P}\left(\tilde{W} \leqslant t\left(\frac{\operatorname{Var}\left(\Lambda_{n}\right)}{\operatorname{Var}\left(\Lambda_{n}^{\prime}\right)}\right)^{1 / 2}\right)-\Phi\left(t\left(\frac{\operatorname{Var}\left(\Lambda_{n}\right)}{\operatorname{Var}\left(\Lambda_{n}^{\prime}\right)}\right)^{1 / 2}\right)\right| \\
\quad+\sup _{t}\left|\Phi\left(t\left(\frac{\operatorname{Var}\left(\Lambda_{n}\right)}{\operatorname{Var}\left(\Lambda_{n}^{\prime}\right)}\right)^{1 / 2}\right)-\Phi(t)\right| .
\end{array}
$$

As previously discussed, for $n$ large, $\operatorname{Var}\left(\Lambda_{n}^{\prime}\right) \geqslant \operatorname{Var}\left(\Lambda_{n}\right) / 2 \geqslant 0.5$, so by (SA.1.13), there exists $C^{\prime}>0$ such that for all $n>0$,

$$
\left|t\left(\frac{\operatorname{Var}\left(\Lambda_{n}\right)}{\operatorname{Var}\left(\Lambda_{n}^{\prime}\right)}\right)^{1 / 2}-t\right| \leqslant|t|\left|\frac{\operatorname{Var}\left(\Lambda_{n}\right)}{\operatorname{Var}\left(\Lambda_{n}^{\prime}\right)}-1\right| \leqslant C^{\prime} n^{-2}|t| .
$$

Then since $\Phi(\cdot)$ is Lipschitz, there exists $C^{\prime \prime}>0$ such that

$$
\sup _{t}\left|\Phi\left(t\left(\frac{\operatorname{Var}\left(\Lambda_{n}\right)}{\operatorname{Var}\left(\Lambda_{n}^{\prime}\right)}\right)^{1 / 2}\right)-\Phi(t)\right| \leqslant C^{\prime \prime} n^{-2} .
$$

Combined with (SA.1.17) and (SA.1.18), we obtain

$$
\sup _{t}\left|\mathbf{P}\left(\operatorname{Var}\left(\Lambda_{n}\right)^{-1 / 2}\left(\Lambda_{n}-\mathbf{E}\left[\Lambda_{n}\right]\right) \leqslant t\right)-\Phi(t)\right| \leqslant C(\log n)^{d q} n \operatorname{Var}\left(\Lambda_{n}\right)^{-q / 2}+O\left(n^{-2}\right) .
$$

By (SA.1.12) and (SA.1.13), $\operatorname{Var}\left(\Lambda_{n}\right)=O\left(n \rho_{n}^{d(p+2) / p}\right)$, so the first term on the righthand side dominates the second term, and (SA.1.3) follows.

Lemma SA.1.3. (SA.1.8) is true.

Proof. This is a consequence of the spatial independence property of Poisson point processes (e.g. Last and Penrose, 2017, Theorem 5.2). Here we provide an elementary proof from first principles. Let $N(S)=\left|\left\{i \in \mathcal{N}_{N_{n}}: X_{i} \in S\right\}\right|$. Let $\boldsymbol{T}_{S}=\left(T_{i}: X_{i} \in\right.$ $S), \boldsymbol{T}_{m}=\left(T_{i}\right)_{i=1}^{m}, \boldsymbol{T}_{m, \ldots, n}=\left(T_{i}\right)_{i=m}^{n}$, and similarly define $\boldsymbol{X}_{m}$ and $\boldsymbol{X}_{m, \ldots, n}$. First consider simple case where $S_{1}, S_{2}$ partition $\operatorname{supp}(f)$. For any $\mathcal{T}_{m} \subseteq \operatorname{supp}\left(\boldsymbol{T}_{m}\right)$ and 


\section{LEUNG AND MOON}

$\mathcal{Z}_{m} \subseteq \operatorname{supp}\left(\boldsymbol{\zeta}_{m}\right)$

$$
\begin{gathered}
\mathbf{P}\left(N\left(S_{1}\right)=m_{1}, \boldsymbol{T}_{S_{1}} \in \mathcal{T}_{m_{1}}, \boldsymbol{\zeta}_{S_{1}} \in \mathcal{Z}_{m_{1}}, N\left(S_{2}\right)=m_{2}, \boldsymbol{T}_{S_{2}} \in \mathcal{T}_{m_{2}}, \boldsymbol{\zeta}_{S_{2}} \in \mathcal{Z}_{m_{2}}\right) \\
=\mathbf{P}\left(N_{n}=m\right) \mathbf{P}\left(N\left(S_{1}\right)=m_{1}, \boldsymbol{T}_{S_{1}} \in \mathcal{T}_{m_{1}}, \boldsymbol{\zeta}_{S_{1}} \in \mathcal{Z}_{m_{1}}, N\left(S_{2}\right)=m_{2},\right. \\
\left.\boldsymbol{T}_{S_{2}} \in \mathcal{T}_{m_{2}}, \boldsymbol{\zeta}_{S_{2}} \in \mathcal{Z}_{m_{2}} \mid N_{n}=m\right) \\
=\frac{e^{-n} n^{m}}{m !} \frac{m !}{m_{1} ! m_{2} !} \mathbf{P}\left(\boldsymbol{X}_{m_{1}} \in S_{1}, \boldsymbol{T}_{m_{1}} \in \mathcal{T}_{m_{1}}, \boldsymbol{\zeta}_{m_{1}} \in \mathcal{Z}_{m_{1}}, \boldsymbol{X}_{m_{1}+1, \ldots, n} \in S_{2},\right. \\
\left.\boldsymbol{T}_{m_{1}+1, \ldots, n} \in \mathcal{T}_{m_{2}}, \boldsymbol{\zeta}_{m_{1}+1, \ldots, n} \in \mathcal{Z}_{m_{2}}\right) \\
=\frac{e^{-n \mathbf{P}\left(X_{1} \in S_{1}\right) n^{m_{1}}}}{m_{1} !} \mathbf{P}\left(\boldsymbol{X}_{m_{1}} \in S_{1}, \boldsymbol{T}_{m_{1}} \in \mathcal{T}_{m_{1}}, \boldsymbol{\zeta}_{m_{1}} \in \mathcal{Z}_{m_{1}}\right) \\
\times \frac{e^{-n \mathbf{P}\left(X_{1} \in S_{2}\right)} n^{m_{2}}}{m_{2} !} \mathbf{P}\left(\boldsymbol{X}_{m_{2}} \in S_{2}, \boldsymbol{T}_{m_{2}} \in \mathcal{T}_{m_{2}}, \boldsymbol{\zeta}_{m_{2}} \in \mathcal{Z}_{m_{2}}\right) .
\end{gathered}
$$

The second line is true because types and random-utility shocks are identically distributed across nodes. The last line is true because disjoint sets of nodes have independent types and random-utility shocks, and $S_{1}, S_{2}$ partition $\operatorname{supp}(f)$.

We perform similar calculations for the marginal distribution:

$$
\begin{aligned}
& \mathbf{P}\left(N\left(S_{1}\right)=m_{1}, \boldsymbol{T}_{S_{1}} \in \mathcal{T}_{m_{1}}, \boldsymbol{\zeta}_{S_{1}} \in \mathcal{Z}_{m_{1}}\right)=\sum_{m \geqslant m_{1}} \mathbf{P}\left(N_{n}=m\right) \mathbf{P}\left(N\left(S_{1}\right)=m_{1},\right. \\
& \left.\boldsymbol{T}_{S_{1}} \in \mathcal{T}_{m_{1}}, \boldsymbol{\zeta}_{S_{1}} \in \mathcal{Z}_{m_{1}}, N\left(S_{1}^{c}\right)=m-m_{1} \mid N_{n}=m\right) \\
& =\sum_{m \geqslant m_{1}} \frac{e^{-n} n^{m}}{m !} \frac{m !}{m_{1} !\left(m-m_{1}\right) !} \mathbf{P}\left(\boldsymbol{X}_{m_{1}} \in S_{1}, \boldsymbol{T}_{m_{1}} \in \mathcal{T}_{m_{1}}, \boldsymbol{\zeta}_{m_{1}} \in \mathcal{Z}_{m_{1}}, \boldsymbol{X}_{m_{1}+1, \ldots, n} \in S_{1}^{c}\right) \\
& =\sum_{m \geqslant m_{1}} \frac{e^{-n \mathbf{P}\left(X_{1} \in S_{1}\right)} n^{m_{1}}}{m_{1} !} \mathbf{P}\left(\boldsymbol{X}_{m_{1}} \in S_{1}, \boldsymbol{T}_{m_{1}} \in \mathcal{T}_{m_{1}}, \boldsymbol{\zeta}_{m_{1}} \in \mathcal{Z}_{m_{1}}\right) \\
& \quad \times \frac{e^{-n \mathbf{P}\left(X_{1} \in S_{1}^{c}\right)} n^{m-m_{1}}}{\left.m-\boldsymbol{X}_{m_{1}+1, \ldots, n} \in S_{1}^{c}\right)} \\
& =\frac{e^{-n \mathbf{P}\left(X_{1} \in S_{1}\right) n^{m_{1}}}}{m_{1} !} \mathbf{P}\left(\boldsymbol{X}_{m_{1}} \in S_{1}, \boldsymbol{T}_{m_{1}} \in \mathcal{T}_{m_{1}}, \boldsymbol{\zeta}_{m_{1}} \in \mathcal{Z}_{\left.m_{1}\right)}^{\sum_{m \geqslant m_{1}} \frac{e^{-n \mathbf{P}\left(X_{1} \in S_{1}^{c}\right)}\left(n \mathbf{P}\left(X_{1} \in S_{1}^{c}\right)\right)^{m-m_{1}}}{m-m_{1} !}} .\right.
\end{aligned}
$$


Therefore,

$$
\begin{aligned}
& \mathbf{P}\left(N\left(S_{1}\right)=m_{1}, \boldsymbol{T}_{S_{1}} \in \mathcal{T}_{m_{1}}, \boldsymbol{\zeta}_{S_{1}} \in \mathcal{Z}_{m_{1}}, N\left(S_{2}\right)=m_{2}, \boldsymbol{T}_{S_{2}} \in \mathcal{T}_{m_{2}}, \boldsymbol{\zeta}_{S_{2}} \in \mathcal{Z}_{m_{2}}\right) \\
& \quad=\mathbf{P}\left(N\left(S_{1}\right)=m_{1}, \boldsymbol{T}_{S_{1}} \in \mathcal{T}_{m_{1}}, \boldsymbol{\zeta}_{S_{1}} \in \mathcal{Z}_{m_{1}}\right) \mathbf{P}\left(N\left(S_{2}\right)=m_{2}, \boldsymbol{T}_{S_{2}} \in \mathcal{T}_{m_{2}}, \boldsymbol{\zeta}_{S_{2}} \in \mathcal{Z}_{m_{2}}\right),
\end{aligned}
$$

which proves (SA.1.8) for the simple case where $S_{1}, S_{2}$ partition $\operatorname{supp}(f)$. For the general case, we would only need to repeat the calculations for the joint distribution, now for

$$
\begin{aligned}
\mathbf{P}\left(N\left(S_{1}\right)=m_{1}, \boldsymbol{T}_{S_{1}} \in \mathcal{T}_{m_{1}}, \boldsymbol{\zeta}_{S_{1}} \in \mathcal{Z}_{m_{1}}, N\left(S_{2}\right)=\right. & m_{2}, \boldsymbol{T}_{S_{2}} \in \mathcal{T}_{m_{2}}, \boldsymbol{\zeta}_{S_{2}} \in \mathcal{Z}_{m_{2}}, \\
& \left.N\left(S_{3}\right)=m_{3}, \boldsymbol{T}_{S_{3}} \in \mathcal{T}_{m_{3}}, \boldsymbol{\zeta}_{S_{3}} \in \mathcal{Z}_{m_{3}}\right)
\end{aligned}
$$

where $S_{3}=\operatorname{supp}(f) \backslash\left(S_{1} \cap S_{2}\right)$.

\section{SA.1.2 de-Poissonization}

As in the previous subsection, we assume $d_{\psi}=1$. The next result shows that, given a CLT for the Poissonized model with node set $\mathcal{N}_{N_{n}}$, a CLT also holds for the original "binomial" model of interest with node set $\mathcal{N}_{n}$. The proof follows the argument in Penrose (2007), with modifications to account for differences between our settings, most notably the definition of stabilization. Define $\sigma_{n}^{2}=n^{-1} \operatorname{Var}\left(\sum_{i=1}^{n} \psi_{i}\left(\mathcal{N}_{n}\right)\right)$.

Theorem SA.1.2. Suppose $d_{\psi}=1$. Under Assumptions 5 and $6, \sup _{n} \sigma_{n}^{2}<\infty$, and if additionally $\liminf \operatorname{in}_{n \rightarrow \infty} \sigma_{n}^{2}>0$, then $\liminf _{n \rightarrow \infty} \tilde{\sigma}_{n}^{2}>0$ and

$$
\sigma_{n}^{-1} \frac{1}{\sqrt{n}} \sum_{i=1}^{n}\left(\psi_{i}\left(\mathcal{N}_{n}\right)-\mathbf{E}\left[\psi_{i}\left(\mathcal{N}_{n}\right)\right]\right) \stackrel{d}{\longrightarrow} \mathcal{N}(0,1) .
$$

The proof is stated at the end of this subsection and uses the next four lemmas. Lemma SA.1.4 establishes an asymptotic equivalence between $\boldsymbol{X}_{n}$ and $\boldsymbol{X}_{N_{n}}$ and is taken directly from Penrose and Yukich (2003). Lemma SA.1.5 proves that node statistics of two nodes whose positions are a fixed distance apart are asymptotically uncorrelated. Lemma SA.1.6 draws on Lemma SA.1.5 to show that add-one costs of two nodes at a fixed distance apart are asymptotically uncorrelated. Finally, Lemma SA.1.7 shows that various moments are uniformly bounded. 


\section{LEUNG AND MOON}

Remark SA.1.2 (Related Literature). These results are adaptations of lemmas in Penrose (2007), so we compare and contrast our setup with his. First, the differences discussed in Remark SA.1.1 persist here. Second, the proofs of Lemmas SA.1.5 and SA.1.6 need some modification because our setups differ, most notably the definition of stabilization. Third, to prove the lemmas, we define a coupling that follows the ideas in Penrose (2007) and Penrose and Yukich (2003). The details are different because we need to accommodate random-utility shocks $\zeta_{i j}$ and differences in notation. Fourth, Penrose considers a generalization of $\psi(\cdot)$ that is a measure in that it takes as an argument a subset of $\mathbb{R}^{d}$. This generalization is unnecessary for our purposes.

The lemmas utilize the following coupling. The essential idea follows Penrose and Yukich (2003), p. 298, but the formal details are different. As in $\S 2.2$, define i.i.d. types $\left\{\left(X_{i}, Z_{i}\right)\right\}_{i \in \mathbb{N}}$ and i.i.d. random-utility shocks $\left\{\zeta_{i j}: i \neq j,\{i, j\} \subseteq \mathbb{N}\right\}$, with the two sets mutually independent. For any $n \in \mathbb{N}$, let $N_{n}^{\prime}$ be an independent copy of $N_{n}$ (defined in $§ 3.2$ ), independent of all other primitives.

- For any $m \in \mathbb{N}$, redefine

$$
\boldsymbol{X}_{m}=\left(X_{5}, X_{7}, X_{9}, \ldots, X_{2 m+3}\right) .
$$

This clearly has the same distribution as the more conventional-looking array $\left(X_{1}, X_{2}, \ldots, X_{m}\right)$. We will see shortly below why we use only odd labels, and we discuss in Remark SA.1.4 why we skip nodes $1, \ldots, 4$.

- Fix any $x, y \in \mathbb{R}^{d}$. Let $F_{x}$ be the set of points in $\mathbb{R}^{d}$ closer to $x$ than $y$ and $F_{y}$ the remaining set of points (break ties arbitrarily). Let $\mathcal{P}_{n f}^{*, x}$ be the restriction of $\tilde{\boldsymbol{X}}_{N_{n}} \equiv\left(X_{5}, X_{7}, X_{9}, \ldots, X_{2 N_{n}+3}\right)$ to $F_{x}$. Let $\mathcal{Q}_{n f}^{*, x}$ be the restriction of $\tilde{\boldsymbol{X}}_{N_{n}^{\prime}} \equiv$ $\left(X_{6}, X_{8}, X_{10} \ldots, X_{2 N_{n}^{\prime}+4}\right)$ to $F_{y}$. Construct $\mathcal{P}_{n f}^{x}$ by concatenating the two vectors $\mathcal{P}_{n f}^{*, x}$ and $\mathcal{Q}_{n f}^{*, x}$. As the notation suggests, for any $n$, the set consisting of the components of $\mathcal{P}_{n f}^{x}$ has the same distribution as an inhomogeneous Poisson point process on $\mathbb{R}^{d}$ with intensity function $n f(\cdot)$. This follows from Poisson superposition (Last and Penrose, 2017, Theorem 3.3) since $F_{x}$ and $F_{y}$ partition $\mathbb{R}^{d}$. Hence, $\mathcal{P}_{n f}^{x}$ has the same distribution as $\boldsymbol{X}_{N_{n}}$ used in $\S$ SA.1.1.

- Similarly, let $\mathcal{P}_{n f}^{*, y}$ be the restriction of $\tilde{\boldsymbol{X}}_{N_{n}}$ to $F_{y}$ and $\mathcal{Q}_{n f}^{*, y}$ the restriction of $\tilde{\boldsymbol{X}}_{N_{n}^{\prime}}$ to $F_{x}$. Construct $\mathcal{P}_{n f}^{y}$ by concatenating the two vectors $\mathcal{P}_{n f}^{*, y}$ and $\mathcal{Q}_{n f}^{*, y}$. 


\section{Normal Approximation in Large Network Models}

Then $\mathcal{P}_{n f}^{y}$ has the same distribution as $\boldsymbol{X}_{N_{n}}$.

The point of this construction is that, by switching the odds and evens on the two half-sets $F_{x}$ and $F_{y}$, we have $\mathcal{P}_{n f}^{x} \Perp \mathcal{P}_{n f}^{y}$. As a consequence, we obtain the following independence property, which can be established following the proof of Lemma SA.1.3. For any $S_{1}, S_{2} \subseteq \mathbb{R}^{d}$ with $S_{1} \cap S_{2}=\varnothing$, define $\mathcal{N}_{x}\left(S_{1}\right)=\left\{k \in \mathbb{N}: X_{k} \in \mathcal{P}_{n f}^{x} \cap S_{1}\right\}$ and $\mathcal{N}_{y}\left(S_{2}\right)$ similarly. Then

$$
\left\{\left(X_{i}, X_{j}, Z_{i}, Z_{j}, \zeta_{i j}\right): i, j \in \mathcal{N}_{x}\left(S_{1}\right)\right\} \Perp\left\{\left(X_{i}, X_{j}, Z_{i}, Z_{j}, \zeta_{i j}\right): i, j \in \mathcal{N}_{y}\left(S_{2}\right)\right\}
$$

This is similar to (SA.1.8) but using two "interleaved" Poisson processes $\mathcal{P}_{n f}^{x}$ and $\mathcal{P}_{n f}^{y}$. The interleaving idea of taking two different "halves" on $F_{x}$ and $F_{y}$ of two independent Poisson processes (in our case, the odds and the evens) is taken from Penrose and Yukich (2003). Our construction only differs by building the two processes from the odd and even elements of the same countable set of random vectors.

Remark SA.1.3. This construction will be used to show that the node statistic of a node positioned at $x$ is asymptotically uncorrelated with that of a node positioned at $y$, for any fixed $x, y \in \mathbb{R}^{d}$ (Lemma SA.1.5). Intuitively, by stabilization, $x$ 's statistic is primarily determined by nodes near $x$ and likewise for $y$, and by Lemma SA.1.4 and stabilization, nodes near $x$ will be given by $\mathcal{P}_{n f}^{x}$ and nodes near $y$ by $\mathcal{P}_{n f}^{y}$, which are independent. Note this does not mean node statistics are essentially i.i.d. because in the continuum limit, there are many nodes near $x$ and many near $y$.

For any $x, y \in \mathbb{R}^{d}, r>0$, and $\boldsymbol{X} \subseteq \mathbb{R}^{d}$, let $\tau_{x, r} y=x+r^{-1}(y-x)$ and $\tau_{x, r} \boldsymbol{X}=$ $\left\{x+r^{-1}(y-x): y \in \boldsymbol{X}\right\}$. This operator dilates the point set $\boldsymbol{X}$ relative to point $x$, whereas in the main text, we instead use $r^{-1} \boldsymbol{X}=\tau_{\mathbf{0}, r} \boldsymbol{X}$, which only dilates $\boldsymbol{X}$ relative to the origin. For technical reasons, it will be convenient to consider dilations relative to other locations. The next coupling lemma shows that the "binomial process" $\tau_{x, r} \boldsymbol{X}_{n}$ and Poisson process $\tau_{x, r} \mathcal{P}_{n f}^{x}$ coincide on neighborhoods of $x$ (and likewise for $y$ ).

Lemma SA.1.4. For any $R>0, x, y \in \mathbb{R}^{d}$, and sequence $\left\{\ell_{n}\right\}_{n \in \mathbb{N}}$ with $\ell_{n} / n \rightarrow 1$,

$$
\mathbf{P}\left(\tau_{x, r_{n}} \boldsymbol{X}_{\ell_{n}} \cap B(x, R)=\tau_{x, r_{n}} \mathcal{P}_{n f}^{x} \cap B(x, R)\right) \rightarrow 1
$$

Proof. This is Lemma 3.1 of Penrose and Yukich (2003). 


\section{LEUNG AND MOON}

The next lemma utilizes nodes $1, \ldots, 4$, which were omitted from the coupling construction above. To state the result and its proof succinctly, we define more compact notation. For $m \in \mathbb{N}$, redefine

$$
\mathcal{N}_{m}=\{5,7,9 \ldots, 2 m+3\}
$$

mirroring the definition of $\boldsymbol{X}_{m}$ from (SA.1.19). For $x \in \mathbb{R}^{d}, J \subseteq \mathbb{N}, i \in J$, let

$$
\psi_{i, x}(J)=\psi\left(i, \tau_{x, r_{n}} \boldsymbol{T}_{J}, \boldsymbol{\zeta}_{J}, \lambda_{|J|}\left(\tau_{x, r_{n}} \boldsymbol{T}_{J}, \boldsymbol{\zeta}_{J}\right)\right)
$$

where $\tau_{x, r_{n}} \boldsymbol{T}_{J}=\left(\left(\tau_{x, r_{n}} X_{i}, Z_{i}\right)\right)_{i \in J}$ and $\boldsymbol{\zeta}_{J}=\left(\zeta_{i j}\right)_{i \neq j ; i, j \in J}$. Thus, for $x=\mathbf{0}, \psi_{i, x}(J)=$ $\psi_{i}(J)$ defined in $(9)$.

For any $H \subseteq \mathbb{N}, \psi_{i, x}\left(\mathcal{N}_{m} \cup H\right)$ corresponds to $i$ 's node statistic in the model with node set $\mathcal{N}_{m} \cup H$ and positions dilated by $r_{n}^{-1}$ relative to $x$. Recall from (SA.1.20) the definition of $\mathcal{N}_{x}\left(S_{1}\right)$, and abbreviate $\mathcal{N}_{x} \equiv \mathcal{N}_{x}\left(\mathbb{R}^{d}\right)$. Then for $m \in\{x, y\}, \psi_{i, x}\left(\mathcal{N}_{m} \cup H\right)$ corresponds to $i$ 's statistic under a Poissonized model with a random number of nodes, whereas when $m \in \mathbb{N}$, it corresponds to the usual "binomial" model with $m$ nodes. Accordingly, in the next lemma, the vector $V_{1}(x, y)$ only involves binomial models, whereas $V_{2}(x, y)$ only involves Poissonized models.

Lemma SA.1.5. Let $\left\{\ell_{n}\right\}_{n \in \mathbb{N}}$ and $\left\{m_{n}\right\}_{n \in \mathbb{N}}$ be sequences of natural numbers such that $\ell_{n} / n$ and $m_{n} / n$ tend to one. For any $x, y \in \operatorname{supp}(f)$, define

$$
\begin{aligned}
V_{1}(x, y)= & \left(\psi_{1, x}\left(\mathcal{N}_{\ell_{n}} \cup\{1\}\right), \psi_{1, x}\left(\mathcal{N}_{\ell_{n}} \cup\{1,2\}\right), \psi_{1, x}\left(\mathcal{N}_{\ell_{n}} \cup\{1,3\}\right),\right. \\
& \psi_{1, x}\left(\mathcal{N}_{\ell_{n}} \cup\{1,2,3\}\right), \psi_{1, x}\left(\mathcal{N}_{m_{n}} \cup\{1\}\right), \psi_{1, x}\left(\mathcal{N}_{m_{n}} \cup\{1,2\}\right), \\
& \psi_{1, x}\left(\mathcal{N}_{m_{n}} \cup\{1,2,3\}\right), \psi_{2, y}\left(\mathcal{N}_{m_{n}} \cup\{2\}\right), \psi_{2, y}\left(\mathcal{N}_{m_{n}} \cup\{1,2\}\right), \\
& \left.\psi_{2, y}\left(\mathcal{N}_{m_{n}} \cup\{1,2,3\}\right), \psi_{2, y}\left(\mathcal{N}_{m_{n}} \cup\{1, \ldots, 4\}\right)\right) \quad \text { and } \\
V_{2}(x, y)= & \psi_{1, x}\left(\mathcal{N}_{x} \cup\{1\}\right), \psi_{1, x}\left(\mathcal{N}_{x} \cup\{1\}\right), \psi_{1, x}\left(\mathcal{N}_{x} \cup\{1,3\}\right), \\
& \psi_{1, x}\left(\mathcal{N}_{x} \cup\{1,3\}\right), \psi_{1, x}\left(\mathcal{N}_{x} \cup\{1\}\right), \psi_{1, x}\left(\mathcal{N}_{x} \cup\{1\}\right), \\
& \psi_{1, x}\left(\mathcal{N}_{x} \cup\{1,3\}\right), \psi_{2, y}\left(\mathcal{N}_{y} \cup\{2\}\right), \psi_{2, y}\left(\mathcal{N}_{y} \cup\{2\}\right), \\
& \left.\psi_{2, y}\left(\mathcal{N}_{y} \cup\{2\}\right), \psi_{2, y}\left(\mathcal{N}_{y} \cup\{2,4\}\right)\right) .
\end{aligned}
$$

Also define $\mathcal{F}(w, x, y, z)=\left\{X_{1}=x, X_{2}=y, X_{3}=x+r_{n} z, X_{4}=y+r_{n} w\right\}$ for 


\section{Normal Approximation in Large Network Models}

$w, z \in \operatorname{supp}(f)$. Under Assumption 5, for any $\epsilon>0$,

$$
\mathbf{P}\left(\left\|V_{1}(x, y)-V_{2}(x, y)\right\|>\epsilon \mid \mathcal{F}(w, x, y, z)\right) \rightarrow 0
$$

where $\|\cdot\|$ denotes the component-wise maximum. Furthermore, the terms in $V_{2}(x, y)$ involving $x$ are independent of the terms involving y by (SA.1.20).

Remark SA.1.4. In this lemma, we have four nodes of interest: two "focal" nodes 1,2 and two "ancillary" nodes 3,4 . The conditioning event $\mathcal{F}(w, x, y, z)$ says that 3 is local to 1 in terms of position (since $r_{n} \rightarrow 0$ ), 4 is local to 2 , and 1 and 2 are a fixed distance $\|x-y\|$ apart and hence non-local. The lemma shows that, in various models with different node sets, node 1's statistic is asymptotically uncorrelated with node 2's statistic since all terms in $V_{2}(x, y)$ involving node 1 are independent of those involving node 2 by the coupling construction. Node 1's statistic only asymptotically depends on 1,3 and not 2,4 (and vice versa for node 2). Note that the labels of these nodes are arbitrary since node statistics are identically distributed.

The purpose of initially holding out nodes $1, \ldots, 4$ in the construction of $\boldsymbol{X}_{m}$ and $\mathcal{N}_{m}$ is that, in the Poissonized model, the number of nodes $N_{n}$ is zero with positive probability. Hence, if we had instead defined $\mathcal{N}_{m} \equiv\{1,3,5, \ldots, 2 m-1\}$, then $\mathcal{N}_{N_{n}}$ could be empty with positive probability, in which case the conditioning event is null. Our construction ensures $\mathcal{N}_{m} \cup H$ is always nonempty for $m \in\{x, y\}$ and $H$ a nonempty subset of $\{1, \ldots, 4\}$.

Proof of Lemma SA.1.5. This lemma is analogous to Lemma 3.7 of Penrose (2007) and Lemma 3.2 of Penrose and Yukich (2003), but the arguments need modification for reasons discussed in Remark SA.1.2. The key step is establishing an analog of (3.4) in the latter reference, which is accomplished in (SA.1.25) below.

We will only prove convergence of arguably the most complicated of the eleven components in $V_{1}(x, y)$ and $V_{2}(x, y)$, namely that for any $\epsilon>0$,

$$
\mathbf{P}\left(\left\|\psi_{2, y}\left(\mathcal{N}_{m_{n}} \cup\{1, \ldots, 4\}\right)-\psi_{2, y}\left(\mathcal{N}_{y} \cup\{2,4\}\right)\right\|>\epsilon \mid \mathcal{F}(w, x, y, z)\right) \rightarrow 0 .
$$

Convergence of the other components follows from the same arguments. 


\section{LEUNG AND MOON}

Condition throughout on $\mathcal{F}(w, x, y, z)$. Define for any $R>0$

$$
\mathcal{E}_{n}(R)=\left\{\tau_{y, r_{n}} \boldsymbol{X}_{m_{n}} \cap B(y, R)=\tau_{y, r_{n}} \mathcal{P}_{n f}^{y} \cap B(y, R)\right\}
$$

and let

$$
\begin{aligned}
\boldsymbol{R}_{2}^{*}=\max \left\{\boldsymbol{R}\left(2, \tau_{y, r_{n}} \boldsymbol{T}_{\mathcal{N}_{m_{n}} \cup\{1, \ldots, 4\}}, \boldsymbol{\zeta}_{\mathcal{N}_{m_{n}} \cup\{1, \ldots, 4\}}, \lambda_{m_{n}+4}\right),\right. \\
\\
\left.\quad \boldsymbol{R}\left(2, \tau_{y, r_{n}} \boldsymbol{T}_{\mathcal{N}_{y} \cup\{2,4\}}, \boldsymbol{\zeta}_{\mathcal{N}_{y} \cup\{2,4\}}, \lambda_{\left|\mathcal{N}_{y}\right|+2}\right)\right\} .
\end{aligned}
$$

The latter is the larger of the radii of stabilization (Definition 1) corresponding to the node statistics $\psi_{2, y}\left(\mathcal{N}_{m_{n}} \cup\{1, \ldots, 4\}\right)$ and $\psi_{2, y}\left(\mathcal{N}_{y} \cup\{2,4\}\right)$, respectively.

For any $R>0$, define $\mathcal{N}(y, R)=\left\{j \in \mathbb{N}: \tau_{y, r_{n}} X_{j} \in B(y, R)\right\}$, the set of nodes whose dilated positions lie within the $R$-ball of $y$. This is analogous to the definition of $\mathcal{N}_{n}\left(\mathcal{B}_{i}\right)$ in $\S 3$. By Definition 1 , if $y=\mathbf{0}$, then under the event $\left\{R>\boldsymbol{R}_{2}^{*}\right\}$,

$$
\psi_{2, y}\left(\mathcal{N}_{m_{n}} \cup\{1, \ldots, 4\}\right)=\psi_{2, y}\left(\left(\mathcal{N}_{m_{n}} \cup\{1, \ldots, 4\}\right) \cap \mathcal{N}(y, R)\right)
$$

By (8), this also holds for $y \neq \mathbf{0}$ (also see (SA.1.26) below).

Recall that under $\mathcal{F}(w, x, y, z)$, the positions of nodes 1,3 , and 4 are $x, x+r_{n} z$, and $y+r_{n} w$, respectively. Since $\tau_{y, r_{n}}\left(\left\{x, x+r_{n} z, y+r_{n} w\right\}\right)=\left\{y+r_{n}^{-1}(x-y), y+\right.$ $\left.r_{n}^{-1}\left(x+r_{n} z-y\right), w+y\right\}$, the intersection of this set and $B(y, R)$ is $\{w+y\}$ for $n, R$ sufficiently large. Then

$$
(\mathrm{SA} .1 .23)=\psi_{2, y}\left(\left(\mathcal{N}_{m_{n}} \cup\{2,4\}\right) \cap \mathcal{N}(y, R)\right)
$$

for such $n, R$ under $\left\{R>\boldsymbol{R}_{2}^{*}\right\}$ (and the conditioning event). For the previous choice of $n, R$, under the event $\mathcal{E}_{n}(R) \cap\left\{R>\boldsymbol{R}_{2}^{*}\right\}$,

$$
(\mathrm{SA} .1 .24)=\psi_{2, y}\left(\left(\mathcal{N}_{y} \cup\{2,4\}\right) \cap \mathcal{N}(y, R)\right)=\psi_{2, y}\left(\mathcal{N}_{y} \cup\{2,4\}\right)
$$

where the first equality uses the event $\mathcal{E}_{n}(R)$, which allows us to replace the "binomial" model with the "Poissonized" model, and the second equality follows from the 


\section{Normal Approximation in Large Network Models}

argument for (SA.1.23). Therefore, by the law of total probability,

$$
\begin{array}{r}
\mathbf{P}\left(\left\|\psi_{2, y}\left(\mathcal{N}_{m_{n}} \cup\{1, \ldots, 4\}\right)-\psi_{2, y}\left(\mathcal{N}_{y} \cup\{2,4\}\right)\right\|>\epsilon \mid \mathcal{F}(w, x, y, z)\right) \\
\leqslant \mathbf{P}\left(\mathcal{E}_{n}(R)^{c}\right)+\mathbf{P}\left(\boldsymbol{R}_{2}^{*}>R\right),
\end{array}
$$

where $\mathcal{E}_{n}(R)^{c}$ is the complement of $\mathcal{E}_{n}(R)$. It remains to show that the right-hand side can be made arbitrarily small by choosing $n, R$ large enough.

We first claim that by Assumption 5, $\boldsymbol{R}_{2}^{*}=O_{p}(1)$. This is not quite immediate from the assumption because, for instance, $\boldsymbol{R}_{2}^{*}$ is defined in terms of the point sets $\tau_{y, r_{n}} \boldsymbol{X}_{m_{n}}$ and $\tau_{y, r_{n}} \mathcal{P}_{n f}^{y}$ rather than $r_{n}^{-1} \boldsymbol{X}_{m_{n}}=\tau_{\mathbf{0}, r_{n}} \boldsymbol{X}_{m_{n}}$ and $r_{n}^{-1} \mathcal{P}_{n f}^{y}=\tau_{\mathbf{0}, r_{n}} \mathcal{P}_{n f}^{y}$ as in the assumption. However, by (8),

$$
\begin{aligned}
\psi\left(i, \tau_{z, r_{n}} \boldsymbol{T}_{m}, \boldsymbol{\zeta}_{m}, \lambda_{m}\right) & \equiv \psi\left(i, \tau_{z, r_{n}} \boldsymbol{T}_{m}, \boldsymbol{\zeta}_{m}, \lambda_{m}\left(\tau_{z, r_{n}} \boldsymbol{T}_{m}, \boldsymbol{\zeta}_{m}\right)\right) \\
& =\psi\left(i, \tau_{z^{\prime}, r_{n}} \boldsymbol{T}_{m}, \boldsymbol{\zeta}_{m}, \lambda_{m}\right) \quad \forall z, z^{\prime} \in \mathbb{R}^{d}, i \in \mathcal{N}_{m}, m \in \mathbb{N}
\end{aligned}
$$

Hence, picking $z=y$ and $z^{\prime}=\mathbf{0}$, we have

$\psi\left(2, \tau_{y, r_{n}} \boldsymbol{T}_{\mathcal{N}_{m_{n}} \cup\{1, \ldots, 4\}}, \boldsymbol{\zeta}_{\mathcal{N}_{m_{n}} \cup\{1, \ldots, 4\}}, \lambda_{m_{n}+4}\right)=\psi\left(2, \tau_{\mathbf{0}, r_{n}} \boldsymbol{T}_{\mathcal{N}_{m_{n}} \cup\{1, \ldots, 4\}}, \boldsymbol{\zeta}_{\mathcal{N}_{m_{n}} \cup\{1, \ldots, 4\}}, \lambda_{m_{n}+4}\right)$.

Consequently,

$\boldsymbol{R}\left(2, \tau_{y, r_{n}} \boldsymbol{T}_{\mathcal{N}_{m_{n}} \cup\{1, \ldots, 4\}}, \boldsymbol{\zeta}_{\mathcal{N}_{m_{n}} \cup\{1, \ldots, 4\}}, \lambda_{m_{n}}\right)=\boldsymbol{R}\left(2, \tau_{\mathbf{0}, r_{n}} \boldsymbol{T}_{\mathcal{N}_{m_{n}} \cup\{1, \ldots, 4\}}, \boldsymbol{\zeta}_{\mathcal{N}_{m_{n}+4} \cup\{1, \ldots, 4\}}, \lambda_{m_{n}+4}\right)$.

Since $r_{n}^{-1} z=\tau_{\mathbf{0}, r_{n}} z$, the right-hand side of the previous display is $O_{p}(1)$ by Assumption 5 . By the same argument,

$\boldsymbol{R}\left(2, \tau_{y, r_{n}} \boldsymbol{T}_{\mathcal{N}_{y} \cup\{2,4\}}, \boldsymbol{\zeta}_{\mathcal{N}_{y} \cup\{2,4\}}, \lambda_{\left|\mathcal{N}_{y}\right|+2}\right)=\boldsymbol{R}\left(2, \tau_{\mathbf{0}, r_{n}} \boldsymbol{T}_{\mathcal{N}_{y} \cup\{2,4\}}, \boldsymbol{\zeta}_{\mathcal{N}_{y} \cup\{2,4\}}, \lambda_{\left|\mathcal{N}_{y}\right|+2}\right)=O_{p}(1)$.

Hence, $\boldsymbol{R}_{2}^{*}=O_{p}(1)$, as desired.

It follows that, for any $\varepsilon>0$, we can choose $R$ large enough such that for all $n$ sufficiently large, $\mathbf{P}\left(\boldsymbol{R}_{2}^{*}>R\right)<\varepsilon / 2$. Furthermore, by Lemma SA.1.4, for any such $R$, we can choose $n$ large enough such that $\mathbf{P}\left(\mathcal{E}_{n}(R)^{c}\right)<\varepsilon / 2$. Combining these facts with (SA.1.25), we obtain (SA.1.22).

The next lemma shows that the add-one costs $\Xi_{\ell}$ and $\Xi_{m}$ defined in (14) are 


\section{LEUnG AND MOON}

asymptotically uncorrelated for $\ell, m$ large.

Lemma SA.1.6. Let $\left\{\nu_{n}\right\}_{n \in \mathbb{N}} \subseteq \mathbb{N}$ be a diverging sequence such that $\nu_{n} / n \rightarrow 0$. Under Assumptions 5 and 6 ,

$$
\sup _{n-\nu_{n} \leqslant \ell<m \leqslant n+\nu_{n}}\left|\mathbf{E}\left[\Xi_{\ell} \Xi_{m}\right]-\mathbf{E}\left[\Xi_{N_{n}}\right]^{2}\right| \rightarrow 0
$$

ProOf. We follow the proof of Lemma 5.1 of Penrose (2007). The main difference is that we use our Lemma SA.1.5 in place of their Lemma 3.7, but there are some additional technical details involved due to differences in our setup. For $i \leqslant m$, let

$\psi_{m}=\psi\left(m, r_{n}^{-1} \boldsymbol{T}_{m}, \boldsymbol{\zeta}_{m}, \lambda_{m}\right), \quad \Delta_{i, m}=\psi\left(i, r_{n}^{-1} \boldsymbol{T}_{m+1}, \boldsymbol{\zeta}_{m+1}, \lambda_{m+1}\right)-\psi\left(i, r_{n}^{-1} \boldsymbol{T}_{m}, \boldsymbol{\zeta}_{m}, \lambda_{m}\right)$

Then

$$
\begin{aligned}
\mathbf{E}\left[\Xi_{\ell} \Xi_{m}\right]=\mathbf{E}\left[\left(\psi_{\ell+1}\right.\right. & \left.\left.+\sum_{i=1}^{\ell} \Delta_{i, \ell}\right)\left(\psi_{m+1}+\sum_{j=1}^{\ell} \Delta_{j, m}+\Delta_{\ell+1, m}+\sum_{k=\ell+2}^{m} \Delta_{k, m}\right)\right] \\
=\mathbf{E}\left[\psi_{\ell+1} \psi_{m+1}\right] & +\ell \mathbf{E}\left[\Delta_{1, \ell} \psi_{m+1}\right]+\ell \mathbf{E}\left[\psi_{\ell+1} \Delta_{1, m}\right]+\ell(\ell-1) \mathbf{E}\left[\Delta_{1, \ell} \Delta_{2, m}\right] \\
& +\ell \mathbf{E}\left[\Delta_{1, \ell} \Delta_{1, m}\right]+\mathbf{E}\left[\psi_{\ell+1} \Delta_{\ell+1, m}\right]+\ell \mathbf{E}\left[\Delta_{1, \ell} \Delta_{\ell+1, m}\right] \\
& +(m-\ell-1) \mathbf{E}\left[\psi_{\ell+1} \Delta_{\ell+2, m}\right]+\ell(m-\ell-1) \mathbf{E}\left[\Delta_{1, \ell} \Delta_{\ell+2, m}\right] .
\end{aligned}
$$

By the law of iterated expectations,

$$
\mathbf{E}\left[\Xi_{N_{n}}\right]=\mathbf{E}\left[\psi_{N_{n}+1}+\sum_{i=1}^{N_{n}} \mathbf{E}\left[\Delta_{i, N_{n}} \mid \boldsymbol{X}_{N_{n}}, N_{n}\right]\right]
$$

This is the expectation of a pointwise sum (i.e. over the points of $\boldsymbol{X}_{N_{n}}$ ) of functionals of a Poisson point process $\boldsymbol{X}_{N_{n}}$. We may then apply Corollary 3.2.3 of Schneider and Weil (2008) and use a change of variables to obtain (recalling the no- 


\section{Normal Approximation in Large Network Models}

tation from Lemma SA.1.5),

$$
\begin{gathered}
\mathbf{E}\left[\Xi_{N_{n}}\right]=\int_{\mathbb{R}^{d}} \mathbf{E}\left[\psi_{1, x}\left(\mathcal{N}_{x} \cup\{1\}\right) \mid X_{1}=x\right] f(x) \mathrm{d} x+\kappa \times \\
\int_{\mathbb{R}^{d}} \int_{\mathbb{R}^{d}} \mathbf{E}\left[\psi_{1, x}\left(\mathcal{N}_{x} \cup\{1,3\}\right)-\psi_{1, x}\left(\mathcal{N}_{x} \cup\{1\}\right) \mid X_{1}=x, X_{3}=x+r_{n} z\right] f(x) f\left(x+r_{n} z\right) \mathrm{d} x \mathrm{~d} z .
\end{gathered}
$$

We need to derive the "limits" of each of the terms in (SA.1.27) and show they match with the corresponding term of $\mathbf{E}\left[\Xi_{N_{n}}\right]^{2}$. We only illustrate the first two terms; the argument for the others is similar.

Term 1. Using (SA.1.26) and $m>\ell \geqslant 1$, the first term $\mathbf{E}\left[\psi_{\ell+1} \psi_{m+1}\right]$ equals,

$$
\int_{\mathbb{R}^{d}} \int_{\mathbb{R}^{d}} \mathbf{E}\left[\psi_{1, x}\left(\mathcal{N}_{\ell} \cup\{1\}\right) \psi_{2, y}\left(\mathcal{N}_{m-1} \cup\{1,2\}\right) \mid X_{1}=x, X_{2}=y\right] f(x) f(y) \mathrm{d} x \mathrm{~d} y .
$$

Since $\ell, m$ are order $n$, by Lemma SA.1.5,

$$
\left|\psi_{1, x}\left(\mathcal{N}_{\ell} \cup\{1\}\right) \psi_{2, y}\left(\mathcal{N}_{m-1} \cup\{1,2\}\right)-\psi_{1, x}\left(\mathcal{N}_{x} \cup\{1\}\right) \psi_{2, y}\left(\mathcal{N}_{y} \cup\{2\}\right)\right| \stackrel{p}{\longrightarrow} 0 \text { (SA.1.29) }
$$

conditional on $X_{1}=x, X_{2}=y$, and $\psi_{1, x}\left(\mathcal{N}_{x} \cup\{1\}\right) \Perp \psi_{2, y}\left(\mathcal{N}_{y} \cup\{2\}\right)$. We then wish to use Assumption 6 and the Vitali convergence theorem to conclude that

$$
\begin{aligned}
& \mid \mathbf{E}\left[\psi_{1, x}\left(\mathcal{N}_{\ell} \cup\{1\}\right) \psi_{2, y}\left(\mathcal{N}_{m-1} \cup\{1,2\}\right) \mid X_{1}=x, X_{2}=y\right] \\
& \quad-\mathbf{E}\left[\psi_{1, x}\left(\mathcal{N}_{x} \cup\{1\}\right) \mid X_{1}=x\right] \mathbf{E}\left[\psi_{2, y}\left(\mathcal{N}_{y} \cup\{2\}\right) \mid X_{2}=y\right] \mid \rightarrow 0 .
\end{aligned}
$$

Supposing this were true, we would have

$$
\left|\mathbf{E}\left[\psi_{\ell+1} \psi_{m+1}\right]-\left(\int_{\mathbb{R}^{d}} \mathbf{E}\left[\psi_{1, x}\left(\mathcal{N}_{x} \cup\{1\}\right) \mid X_{1}=x\right] f(x) \mathrm{d} x\right)^{2}\right| \rightarrow 0,
$$

since $\mathbf{E}\left[\psi_{1, x}\left(\mathcal{N}_{x} \cup\{1\}\right) \mid X_{1}=x\right]=\mathbf{E}\left[\psi_{2, x}\left(\mathcal{N}_{x} \cup\{2\}\right) \mid X_{2}=x\right]$.

A technical issue with (SA.1.30) is that it involves conditional expectations, whereas the Vitali convergence theorem applies to unconditional expectations. However, it is straightforward to rewrite them as unconditional expectations by modifying the coupling construction at the start of $\S$ SA.1.2 as follows. First, let $X_{1}$ and $X_{2}$ be nonrandom with respective values $x$ and $y$. Second, let $Z_{1}$ be independently drawn from 


\section{LEUNG AND MOON}

the conditional distribution of $Z_{1}$ given $X_{1}=x$ and $Z_{2}$ likewise independently drawn from the conditional distribution of $Z_{1}$ given $X_{1}=y$, both independent of all other primitives. All other elements of the coupling remain the same. Then letting $\mathbf{E}_{*}[\cdot]$ denote the expectation with respect to this new coupling and $\mathbf{E}[\cdot]$ the expectation under the original coupling, we have

$$
\begin{aligned}
& \mathbf{E}\left[\psi_{1, x}\left(\mathcal{N}_{\ell} \cup\{1\}\right) \psi_{2, y}\left(\mathcal{N}_{m-1} \cup\{1,2\}\right) \mid X_{1}=x, X_{2}=y\right] \\
& =\mathbf{E}_{*}\left[\psi_{1, x}\left(\mathcal{N}_{\ell} \cup\{1\}\right) \psi_{2, y}\left(\mathcal{N}_{m-1} \cup\{1,2\}\right)\right], \\
& \mathbf{E}\left[\psi_{1, x}\left(\mathcal{N}_{x} \cup\{1\}\right) \mid X_{1}=x\right]=\mathbf{E}_{*}\left[\psi_{1, x}\left(\mathcal{N}_{x} \cup\{1\}\right)\right], \quad \text { and } \\
& \mathbf{E}\left[\psi_{2, y}\left(\mathcal{N}_{y} \cup\{2\}\right) \mid X_{2}=y\right]=\mathbf{E}_{*}\left[\psi_{2, y}\left(\mathcal{N}_{y} \cup\{2\}\right)\right] .
\end{aligned}
$$

By (SA.1.29), Assumption 6, and the Vitali convergence theorem,

$\left|\mathbf{E}_{*}\left[\psi_{1, x}\left(\mathcal{N}_{\ell} \cup\{1\}\right) \psi_{2, y}\left(\mathcal{N}_{m-1} \cup\{1,2\}\right)\right]-\mathbf{E}_{*}\left[\psi_{1, x}\left(\mathcal{N}_{x} \cup\{1\}\right)\right] \mathbf{E}_{*}\left[\psi_{2, y}\left(\mathcal{N}_{y} \cup\{2\}\right)\right]\right| \rightarrow 0$, which implies (SA.1.30) by (SA.1.31).

Term 2. Next consider the second term on the right-hand side of (SA.1.27). Recalling the notation Lemma SA.1.5, for $m>\ell \geqslant 1$,

$$
\begin{aligned}
& \ell \mathbf{E}\left[\Delta_{1, \ell} \psi_{m+1}\right] \\
& =\ell \mathbf{E}\left[\left(\psi\left(1, r_{n}^{-1} \boldsymbol{T}_{\ell+1}, \boldsymbol{\zeta}_{\ell+1}, \lambda_{\ell+1}\right)-\psi\left(1, r_{n}^{-1} \boldsymbol{T}_{\ell}, \boldsymbol{\zeta}_{\ell}, \lambda_{\ell}\right)\right) \psi\left(m+1, r_{n}^{-1} \boldsymbol{T}_{m+1}, \boldsymbol{\zeta}_{m+1}, \lambda_{m+1}\right)\right] \\
& =\ell \mathbf{E}\left[\left(\psi_{1, \mathbf{0}}\left(\mathcal{N}_{\ell-1} \cup\{1,3\}\right)-\psi_{1, \mathbf{0}}\left(\mathcal{N}_{\ell-1} \cup\{1\}\right)\right) \psi_{2, \mathbf{0}}\left(\mathcal{N}_{m-2} \cup\{1,2,3\}\right)\right], \quad(\text { SA.1.32) }
\end{aligned}
$$

using the fact that types and random-utility shocks are identically distributed and (SA.1.21). By (SA.1.26),

$$
\begin{aligned}
(\mathrm{SA} .1 .32) & =\ell \mathbf{E}\left[\left(\psi_{1, X_{1}}\left(\mathcal{N}_{\ell-1} \cup\{1,3\}\right)-\psi_{1, X_{1}}\left(\mathcal{N}_{\ell-1} \cup\{1\}\right)\right) \psi_{2, X_{2}}\left(\mathcal{N}_{m-2} \cup\{1,2,3\}\right)\right] \\
& =\ell \int_{\mathbb{R}^{d}} \int_{\mathbb{R}^{d}} \int_{\mathbb{R}^{d}} \mathbf{E}\left[\left(\psi_{1, x}\left(\mathcal{N}_{\ell-1} \cup\{1,3\}\right)-\psi_{1, x}\left(\mathcal{N}_{\ell-1} \cup\{1\}\right)\right)\right. \\
& \left.\times \psi_{2, y}\left(\mathcal{N}_{m-2} \cup\{1,2,3\}\right) \mid X_{1}=x, X_{2}=y, X_{3}=w\right] f(x) f(y) f(w) \mathrm{d} x \mathrm{~d} y \mathrm{~d} w .
\end{aligned}
$$




\section{Normal Approximation in Large Network Models}

By a change of variables $z=r_{n}^{-1}(w-x)$, the last line equals

$$
\begin{array}{r}
\ell r_{n}^{d} \int_{\mathbb{R}^{d}} \int_{\mathbb{R}^{d}} \int_{\mathbb{R}^{d}} \mathbf{E}\left[\left(\psi_{1, x}\left(\mathcal{N}_{\ell-1} \cup\{1,3\}\right)-\psi_{1, x}\left(\mathcal{N}_{\ell-1} \cup\{1\}\right)\right)\right. \\
\left.\times \psi_{2, y}\left(\mathcal{N}_{m-2} \cup\{1,2,3\}\right) \mid X_{1}=x, X_{2}=y, X_{3}=x+r_{n} z\right] \\
\quad f(x) f(y) f\left(x+r_{n} z\right) \mathrm{d} x \mathrm{~d} y \mathrm{~d} z
\end{array}
$$

Recall the new coupling construction for Term 1 above. We modify this coupling by additionally defining $X_{3}$ as non-random and equal to $x+r_{n} z$ and $Z_{3}$ as an independent draw from the conditional distribution of $Z_{1}$ given $X_{1}=x+r_{n} z$. Then

following the argument for Term 1, by Lemma SA.1.5, Assumption 6, and the Vitali convergence theorem, the absolute difference between the integrand of (SA.1.33) and

$$
\begin{aligned}
\mathbf{E}\left[\psi_{1, x}\left(\mathcal{N}_{x} \cup\{1,3\}\right)-\right. & \left.\psi_{1, x}\left(\mathcal{N}_{x} \cup\{1\}\right) \mid X_{1}=x, X_{3}=x+r_{n} z\right] \\
& \times \mathbf{E}\left[\psi_{2, y}\left(\mathcal{N}_{y} \cup\{2\}\right) \mid X_{2}=y\right] f(x) f(y) f\left(x+r_{n} z\right)
\end{aligned}
$$

is $o(1)$.

To apply the dominated convergence theorem, we show that the integrand of (SA.1.33) is uniformly bounded by an integrable function. We continue to employ the new coupling construction used to establish (SA.1.34). Observe that if

$$
\|z\| \geqslant \boldsymbol{R}\left(1, \tau_{x, r_{n}} \boldsymbol{T}_{\mathcal{N}_{\ell-1} \cup\{1,3\}}, \boldsymbol{\zeta}_{\mathcal{N}_{\ell-1} \cup\{1,3\}}, \lambda_{\ell+1}\right),
$$

then since $X_{1}=x$ and $X_{3}=x+r_{n} z$, we have

$$
r_{n}^{-1}\left\|X_{1}-X_{3}\right\| \geqslant \boldsymbol{R}\left(1, \tau_{x, r_{n}} \boldsymbol{T}_{\mathcal{N}_{\ell-1} \cup\{1,3\}}, \boldsymbol{\zeta}_{\mathcal{N}_{\ell-1} \cup\{1,3\}}, \lambda_{\ell+1}\right) .
$$

By Definition 1, this implies

$$
\psi_{1, x}\left(\mathcal{N}_{\ell-1} \cup\{1,3\}\right)=\psi_{1, x}\left(\mathcal{N}_{\ell-1} \cup\{1\}\right),
$$

in which case the integrand of (SA.1.33) is zero. By Assumption 6 and the Hölder and Minkowski inequalities, there exist $C, \epsilon>0$ such that for $n$ large, the integrand 


\section{LEUNG AND MOON}

of (SA.1.33) is bounded in absolute value by

$$
\begin{aligned}
\left(\left\|\psi_{1, x}\left(\mathcal{N}_{\ell-1} \cup\{1,3\}\right)\right\|_{p}+\left\|\psi_{1, x}\left(\mathcal{N}_{\ell-1} \cup\{1\}\right)\right\|_{p}\right) \cdot\left\|\psi_{2, y}\left(\mathcal{N}_{m-2}, \cup\{1,2,3\}\right)\right\|_{p} \\
\quad \times \mathbf{P}\left(\boldsymbol{R}\left(1, \tau_{x, r_{n}} \boldsymbol{T}_{\mathcal{N}_{\ell-1} \cup\{1,3\}}, \boldsymbol{\zeta}_{\mathcal{N}_{\ell-1} \cup\{1,3\}}, \lambda_{\ell+1}\right)>\|z\|\right)^{1-2 / p} \leqslant C \tau_{b, \epsilon}(\|z\|)^{1-2 / p},
\end{aligned}
$$

where $p$ is defined in Assumption 6 and, for any random variable $W,\|W\|_{p} \equiv$ $\sup _{x, y, z} \mathbf{E}\left[|W|^{p} \mid X_{1}=x, X_{2}=y, X_{3}=z\right]^{1 / p}$. By Assumption 5,

$$
\begin{aligned}
C \ell r_{n}^{d} \int_{\mathbb{R}^{d}} \int_{\mathbb{R}^{d}} \int_{\mathbb{R}^{d}} \tau_{b, \epsilon}(\|z\|)^{1-2 / p} f(x) f(y) f(x & \left.+r_{n} z\right) \mathrm{d} x \mathrm{~d} y \mathrm{~d} z \\
& \leqslant C \kappa \sup _{w} f(w) \int_{\mathbb{R}^{d}} \tau_{b, \epsilon}(\|z\|)^{1-2 / p} \mathrm{~d} z<\infty,
\end{aligned}
$$

which verifies the dominance condition. Therefore, by (SA.1.33), (SA.1.34) and dominated convergence, the following is $o(1)$ :

$$
\begin{gathered}
\mid \ell \mathbf{E}\left[\Delta_{1, \ell} \xi_{m+1}\right]-\kappa \int_{\mathbb{R}^{d}} \mathbf{E}\left[\psi_{2, y}\left(\mathcal{N}_{y} \cup\{2\}\right) \mid X_{2}=y\right] f(y) \mathrm{d} y \quad \times \\
\int_{\mathbb{R}^{d}} \int_{\mathbb{R}^{d}} \mathbf{E}\left[\psi_{1, x}\left(\mathcal{N}_{x} \cup\{1,3\}\right)-\psi_{1, x}\left(\mathcal{N}_{x} \cup\{1\}\right) \mid X_{1}=x, X_{3}=x+r_{n} z\right] f(x) f\left(x+r_{n} z\right) \mathrm{d} x \mathrm{~d} z \mid .
\end{gathered}
$$

The last lemma shows that add-one costs have bounded moments.

Lemma SA.1.7. Let $\left\{\nu_{n}\right\}_{n \in \mathbb{N}} \subseteq \mathbb{N}$ be a diverging sequence such that $\nu_{n} / n \rightarrow 0$. Under Assumptions 5 and 6 , for $\tilde{\sigma}_{n}^{2}$ defined in $\S S A .1 .1$,

$$
\limsup _{n \rightarrow \infty} \sup _{n-\nu_{n} \leqslant m \leqslant n+\nu_{n}} \mathbf{E}\left[\Xi_{m}^{2}\right]<\infty \quad \text { and } \sup _{n} \max \left\{\mathbf{E}\left[\Xi_{N_{n}}\right], \tilde{\sigma}_{n}^{2}\right\}<\infty \text {. }
$$

ProOf. The result for $\mathbf{E}\left[\Xi_{m}^{2}\right]$ follows from the proof of Lemma 5.2 of Penrose (2007). The effort of translating the proof to our notation is the same as what was done for the previous two lemmas, so we omit the argument.

Recall the characterization of $\mathbf{E}\left[\Xi_{N_{n}}\right]$ in (SA.1.28). The first term on the righthand side of this equation is uniformly bounded in $n$ by Assumption 6. For the second term on the right-hand side, note that if $\|z\| \geqslant \boldsymbol{R}\left(1, \tau_{x, r_{n}} \boldsymbol{T}_{\mathcal{N}_{x} \cup\{1,3\}}, \boldsymbol{\zeta}_{\mathcal{N}_{x} \cup\{1,3\}}, \lambda_{\left|\mathcal{N}_{x}\right|+2}\right)$, then since we are conditioning on $X_{1}=x$ and $X_{3}=x+r_{n} z$, we have $r_{n}^{-1} \| X_{1}-$ 


\section{Normal Approximation in Large Network Models}

$X_{3} \| \geqslant \boldsymbol{R}\left(1, \tau_{x, r_{n}} \boldsymbol{T}_{\mathcal{N}_{x} \cup\{1,3\}}, \boldsymbol{\zeta}_{\mathcal{N}_{x} \cup\{1,3\}}, \lambda_{\left|N_{x}\right|+2}\right)$. Hence, by definition of the radius of stabilization, $\psi_{1, x}\left(\mathcal{N}_{x} \cup\{1,3\}\right)=\psi_{1, x}\left(\mathcal{N}_{x} \cup\{1\}\right)$. Therefore, by Assumption 6 and the Hölder and Minkowski inequalities, there exist $C, \epsilon>0$ such that for $n$ sufficiently large, the integrand of the second term on the right-hand side is bounded above in absolute value by

$$
\begin{aligned}
\left(\left\|\psi_{1, x}\left(\mathcal{N}_{x} \cup\{1,3\}\right)\right\|_{p}+\left\|\psi_{1, x}\left(\mathcal{N}_{x} \cup\{1\}\right)\right\|_{p}\right) & f(x) f\left(x+r_{n} z\right) \\
\times & \mathbf{P}\left(\boldsymbol{R}\left(1, \tau_{x, r_{n}} \boldsymbol{T}_{\mathcal{N}_{x} \cup\{1,3\}}, \boldsymbol{\zeta}_{\mathcal{N}_{x} \cup\{1,3\}}, \lambda_{\left|N_{x}\right|+2}\right)>\|z\|\right)^{1-1 / p} \\
& \leqslant C f(x) \sup _{w} f(w) \tau_{p}(\|z\|)^{1-1 / p},
\end{aligned}
$$

where for any random variable $W,\|W\|_{p} \equiv \sup _{x, z} \mathbf{E}\left[|W|^{p} \mid X_{1}=x, X_{3}=z\right]^{1 / p}$. The right-hand side of (SA.1.35) does not depend on $n$ and has a finite integral.

For $\tilde{\sigma}_{n}^{2}$, by the argument for (SA.1.28),

$$
\begin{aligned}
& \tilde{\sigma}_{n}^{2}=\int_{\mathbb{R}^{d}} \mathbf{E}\left[\psi_{1, x}\left(\mathcal{N}_{x} \cup\{1\}\right)^{2} \mid X_{1}=x\right] f(x) \mathrm{d} x \\
& \quad+\kappa \int_{\mathbb{R}^{d}} \int_{\mathbb{R}^{d}}\left(\mathbf{E}\left[\psi_{1, x}\left(\mathcal{N}_{x} \cup\{1,3\}\right) \psi_{1, x}\left(\mathcal{N}_{x} \cup\{1\}\right) \mid X_{1}=x, X_{3}=x+r_{n} z\right]\right. \\
& \left.-\mathbf{E}\left[\psi_{1, x}\left(\mathcal{N}_{x} \cup\{1\}\right) \mid X_{1}=x\right] \mathbf{E}\left[\psi_{3, x}\left(\mathcal{N}_{x+r_{n} z} \cup\{3\}\right) \mid X_{3}=x+r_{n} z\right]\right) f(x) f\left(x+r_{n} z\right) \mathrm{d} x \mathrm{~d} z .
\end{aligned}
$$

The first term is uniformly bounded by Assumption 6. The second term is also uniformly bounded by an argument similar to (SA.1.35). ${ }^{10}$

Proof of Theorem SA.1.2. We largely follow the proof of Theorem 2.12 of Penrose (2003) and Theorem 2.3 in Penrose (2007), although the characteristic function argument at the end is different since we do not derive the limit variance. Abbreviate $\alpha_{n}=\mathbf{E}\left[\Xi_{N_{n}}\right]$ and $\Lambda_{m}=\sum_{i=1}^{m} \psi_{i}\left(\mathcal{N}_{m}\right)$. We first prove that

$$
\mathbf{E}\left[n^{-1}\left(\Lambda_{N_{n}}-\Lambda_{n}-\left(N_{n}-n\right) \alpha_{n}\right)^{2}\right] \rightarrow 0 .
$$

\footnotetext{
${ }^{10}$ For more detail, see steps 1 and 3 of the proof of Lemma H.4 in Leung and Moon (2019).
} 


\section{LEUNG AND MOON}

The left-hand side equals

$$
\begin{aligned}
\sum_{m:|m-n| \leqslant n^{3 / 4}} \mathbf{E}\left[n^{-1}\left(\Lambda_{m}-\Lambda_{n}-(m-n) \alpha_{n}\right)^{2}\right] \mathbf{P}\left(N_{n}=m\right) \\
+\mathbf{E}\left[n^{-1}\left(\Lambda_{N_{n}}-\Lambda_{n}-\left(N_{n}-n\right) \alpha_{n}\right)^{2} \mathbf{1}\left\{\left|N_{n}-n\right|>n^{3 / 4}\right\}\right] .
\end{aligned}
$$

Let $\varepsilon>0$. For $n$ sufficiently large and $m \in\left[n, n+n^{3 / 4}\right]$,

$\mathbf{E}\left[\left(\Lambda_{m}-\Lambda_{n}-(m-n) \alpha_{n}\right)^{2}\right]=\mathbf{E}\left[\left(\sum_{\ell=n}^{m-1}\left(\Xi_{\ell}-\alpha_{n}\right)\right)^{2}\right] \leqslant \varepsilon(m-n)^{2}+\varepsilon n^{1 / 2}(m-n)$,

where the inequality follows from expanding the square and applying Lemmas SA.1.6 and SA.1.7 to the summands. A similar argument also applies to $m \in\left[n-n^{3 / 4}, n\right]$. Then the first term of (SA.1.37) is bounded by

$$
n^{-1} \mathbf{E}\left[\varepsilon\left(N_{n}-n\right)^{2}+\varepsilon n^{1 / 2}\left|N_{n}-n\right|\right] \leqslant n^{-1} \varepsilon\left(n+n^{1 / 2}\left(\mathbf{E}\left|N_{n}-n\right|^{2}\right)^{1 / 2}\right)=2 \varepsilon .
$$

Following the argument in the proof of Theorem 2.3 in Penrose (2007), the second term of (SA.1.37) can be bounded by a constant times

$$
n^{-1} n^{(2 p+1) / p} \mathbf{P}\left(\left|N_{n}-n\right|>n^{3 / 4}\right)^{1-2 / p^{\prime}}
$$

for some $p^{\prime} \in(2, p)$ with $p$ defined in Assumption 6. This argument uses Assumption 6 and Lemma SA.1.2. The previous display tends to zero since the probability decays exponentially with $n$ by Lemma 1.4 of Penrose (2003). This establishes (SA.1.36).

To calculate $n^{-1} \operatorname{Var}\left(\Lambda_{n}\right)$, consider the identity

$$
n^{-1 / 2} \Lambda_{N_{n}}=n^{-1 / 2} \Lambda_{n}+n^{-1 / 2}\left(N_{n}-n\right) \alpha_{n}+n^{-1 / 2}\left(\Lambda_{N_{n}}-\Lambda_{n}-\left(N_{n}-n\right) \alpha_{n}\right) .
$$

The variance of the last term tends to zero by (SA.1.36). The second term on the right-hand side has variance $\alpha_{n}^{2}$ and is independent of the first term by the coupling construction. Therefore,

$$
\frac{1}{n} \operatorname{Var}\left(\Lambda_{N_{n}}\right)=\frac{1}{n} \operatorname{Var}\left(\Lambda_{n}\right)+\alpha_{n}^{2}+o(1) \quad \Rightarrow \quad \sigma_{n}^{2}=\tilde{\sigma}_{n}^{2}-\alpha_{n}^{2}+o(1),
$$

and $\sup _{n} \sigma_{n}^{2}<\infty$ since $\sup _{n} \max \left\{\alpha_{n}, \tilde{\sigma}_{n}^{2}\right\}<\infty$ by Lemma SA.1.7. 


\section{Normal Approximation in Large Network Models}

Since $\sigma_{n}^{2}$ has strictly positive limit infimum by assumption, so does $\tilde{\sigma}_{n}^{2}$. We can then rewrite (SA.1.38) as

$$
n^{-1 / 2} \tilde{\sigma}_{n}^{-1}\left(\Lambda_{N_{n}}-\mathbf{E}\left[\Lambda_{N_{n}}\right]\right)-o_{p}(1)=\frac{\sigma_{n}}{\tilde{\sigma}_{n}} n^{-1 / 2} \sigma_{n}^{-1}\left(\Lambda_{n}-\mathbf{E}\left[\Lambda_{N_{n}}\right]\right)+\frac{\alpha_{n}}{\tilde{\sigma}_{n}} n^{-1 / 2}\left(N_{n}-n\right) .
$$

Let $\tilde{\varphi}_{n}(\cdot)$ be the characteristic function of the left-hand side, $\varphi_{n}(\cdot)$ that of $n^{-1 / 2} \sigma_{n}^{-1}\left(\Lambda_{n}-\right.$ $\left.\mathbf{E}\left[\Lambda_{N_{n}}\right]\right)$, and $\varphi_{p}(\cdot)$ that of $n^{-1 / 2}\left(N_{n}-n\right)$. By independence of $N_{n}$, due to the coupling construction, the characteristic function of the right-hand side of (SA.1.40) is

$$
\varphi_{n}\left(\frac{\sigma_{n}}{\tilde{\sigma}_{n}} x\right) \varphi_{p}\left(\frac{\alpha_{n}}{\tilde{\sigma}_{n}} x\right), \quad \text { implying } \varphi_{n}(y)=\tilde{\varphi}_{n}\left(\frac{\tilde{\sigma}_{n}}{\sigma_{n}} y\right) \varphi_{p}\left(\frac{\alpha_{n}}{\sigma_{n}} y\right)^{-1}
$$

for $y=\sigma_{n} \tilde{\sigma}_{n}^{-1} x$. By Theorem SA.1.1 and the Poisson CLT,

$$
\tilde{\varphi}_{n}(x) \rightarrow e^{-x^{2} / 2} \quad \text { and } \quad \varphi_{p}(x) \rightarrow e^{-x^{2} / 2}
$$

Furthermore, this convergence is uniform over $x$ in a compact set (Klenke, 2013, Theorem 15.23). As previously discussed, $\sup _{n} \max \left\{\alpha_{n}, \tilde{\sigma}_{n}\right\}<\infty$ and $\liminf _{n \rightarrow \infty} \min \left\{\sigma_{n}, \tilde{\sigma}_{n}\right\}>$ 0 . Thus, there exists a compact set $K$ such that for $n$ sufficiently large and $y \in K$, $y \tilde{\sigma}_{n} / \sigma_{n}$ and $y \alpha_{n} / \sigma_{n}$ lie in $K$. Combining these facts,

$$
\varphi_{n}(y)=\frac{\exp \left\{-\frac{\tilde{\sigma}_{n}^{2}}{\sigma_{n}^{2}} \frac{y^{2}}{2}\right\}+o(1)}{\exp \left\{-\frac{\alpha_{n}^{2}}{\sigma_{n}^{2}} \frac{y^{2}}{2}\right\}+o(1)}=\frac{\exp \left\{-\frac{\tilde{\sigma}_{n}^{2}-\alpha_{n}^{2}}{\sigma_{n}^{2}} \frac{y^{2}}{2}\right\}+o(1)}{1+o(1)} \rightarrow e^{-y^{2} / 2}
$$

for any $y \in \mathbb{R}$, where convergence follows from (SA.1.39). Therefore,

$$
n^{-1 / 2} \sigma_{n}^{-1}\left(\Lambda_{n}-\mathbf{E}\left[\Lambda_{N_{n}}\right]\right) \stackrel{d}{\longrightarrow} \mathcal{N}(0,1)
$$

Finally, in the previous expression, we can replace $\mathbf{E}\left[\Lambda_{N_{n}}\right]$ with $\mathbf{E}\left[\Lambda_{n}\right]$ due to (SA.1.36).

\section{SA.2 Auxiliary Lemmas}

The lemmas in this section are used to prove results in $\S$ A.2. The first two show that $\left|\mathfrak{X}_{r_{n}}^{D}(x, z)\right|$ and $\left|\mathfrak{X}_{r_{n}}^{M}(x, z ; K)\right|$ defined in $\S$ A.1 have exponential tails. Let $\mathcal{T}=$ 


\section{LEUNG AND MOON}

$\operatorname{supp}\left(X_{1}, Z_{1}\right)$. For $r>0$ and $\bar{f}=\sup _{x} f(x)$, define $g_{r}^{\alpha}(x, z)=\mathbf{E}\left[\alpha^{\left|\mathfrak{X}_{r}^{D}(x, z)\right|}\right]$ and

$$
\psi_{r}(x, z)=\kappa \bar{f}(1+r) \int_{\mathbb{R}^{d}}\left(\int_{\mathbb{R}^{d z}} p_{1}\left(x, z ; x^{\prime}, z^{\prime}\right)^{2} \mathrm{~d} \Phi^{*}\left(z^{\prime}\right)\right)^{1 / 2} \mathrm{~d} x^{\prime}
$$

Lemma SA.2.1. Under Assumptions 1, 7, and 9, there exists $\alpha>1$ such that, for $r$ sufficiently small, $\sup _{(x, z) \in \mathcal{T}} g_{r}^{\alpha}(x, z)<\infty$.

Proof. By Lemma SA.3.3 of Leung (2020), the conclusion holds if $\inf _{(x, z) \in \mathcal{T}} \psi_{0}(x, z)>$ 0 and $\sup _{(x, z) \in \mathcal{T}} \psi_{0}(x, z)<\infty$. Assumption 1 implies the second inequality, and Assumption 9 implies the first because

$$
\lim _{n \rightarrow \infty} \psi_{r_{n}}(x, z) \geqslant \lim _{n \rightarrow \infty} n \mathbf{E}\left[p_{r_{n}}\left(X_{i}, Z_{i} ; X_{j}, Z_{j}\right) \mid X_{i}=x, Z_{i}=z\right]
$$

Lemma SA.2.2. Define $\bar{g}_{r}^{\alpha}(x, z ; K)=\mathbf{E}\left[\alpha^{\left|\mathfrak{X}_{r}^{M}(x, z ; K)\right|}\right]$. Under Assumption 1, for any $\alpha>1$ and $K \in \mathbb{N}, \sup _{r \leqslant \kappa} \sup _{(x, z) \in \mathcal{T}} \mathbf{E}\left[\bar{g}_{r}^{\alpha}(x, z ; K)\right]<\infty$.

ProOF. Let $\bar{T}_{r}$ be the functional that maps any $h: \mathbb{R}^{d} \times \mathbb{R}^{d_{z}} \rightarrow \mathbb{R}$ to

$$
\left(\bar{T}_{r} h\right)(x, z)=\int_{\mathbb{R}^{d}} \int_{\mathbb{R}^{d z}} h\left(x^{\prime}, z^{\prime}\right) \mathrm{d} \bar{\varphi}_{r}\left(x, z ; x^{\prime}, z^{\prime}\right),
$$

where $\bar{\varphi}_{r}(\cdot)$ is defined in (A.5). A standard branching process argument yields the recursion

$$
\begin{array}{r}
\bar{g}_{r}^{\alpha}(x, z ; K) \\
=\alpha \exp \left\{\kappa \bar{f}(1+r) \int_{\mathbb{R}^{d}} \int_{\mathbb{R}^{d z}}\left(\bar{g}_{r}^{\alpha}\left(x^{\prime}, z^{\prime} ; K-1\right)-1\right) \bar{p}_{r}\left(x ; x^{\prime}\right) \mathrm{d} \Phi^{*}\left(z^{\prime}\right) \mathrm{d} x^{\prime}\right\} \\
=\alpha \exp \left\{\bar{T}_{r}\left(\bar{g}_{r}^{\alpha}(x, z ; K-1)-1\right)\right\} .
\end{array}
$$

See for example the proof of Lemma I.2 of Leung and Moon (2019). Let $\Gamma: g \mapsto$ $\alpha \exp \left\{\bar{T}_{r}(g-1)\right\}$. Then for any $(x, z) \in \mathcal{T}$,

$$
\bar{g}_{r}^{\alpha}(x, z ; K)=\Gamma^{K} \alpha
$$




\section{Normal Approximation in Large Network Models}

by (SA.2.1) and the fact that $\left|\mathfrak{X}_{r}^{M}(x, z ; 0)\right|=1$. Now, $\Gamma \alpha$ equals

$$
\alpha e^{\alpha-1} \exp \left\{\kappa(1+r) \bar{f} \int_{\mathbb{R}^{d}} \int_{\mathbb{R}^{d_{z}}} \bar{p}_{r}\left(x ; x^{\prime}\right) \mathrm{d} \Phi^{*}\left(z^{\prime}\right) \mathrm{d} x^{\prime}\right\} .
$$

By Assumption 1, $\sup _{x} \int_{\mathbb{R}^{d}} \bar{p}_{1}\left(x ; x^{\prime}\right) \mathrm{d} x^{\prime}<\infty$ since $\bar{p}_{1}\left(x ; x^{\prime}\right) \leqslant \bar{\Phi}_{\zeta}\left(\bar{V}^{-1}\left(\left\|x-x^{\prime}\right\|, 0\right)\right)$ decays exponentially in $\left\|x-x^{\prime}\right\|$. Consequently, there exists $C<\infty$ such that, for any $r \leqslant \kappa$, (SA.2.3) is bounded by $\alpha e^{\alpha-1} C$. Likewise, $\Gamma^{2} \alpha<\alpha e^{\alpha e^{\alpha-1} C-1} C<\infty$, and repeating this argument, we obtain $\sup _{(x, z) \in \mathcal{T}} \Gamma^{K} \alpha<\infty$. Combined with (SA.2.2), this proves the claim.

The next lemma establishes an exponential tail bound for $\left|\mathfrak{X}_{r_{n}}^{H}\left(X_{1}, Z_{1} ; K\right)\right|$ defined in (A.7).

Lemma SA.2.3. Under Assumptions 1, 7, and 9, there exists $n_{0} \in \mathbb{N}$ such that

$$
\begin{aligned}
\limsup _{w \rightarrow \infty} w^{-1} \log \beta(w)<0 & \text { for } \\
\beta(w) & =\sup _{n>n_{0}} \sup _{(x, z) \in \mathcal{T}} \mathbf{P}\left(\left|\mathfrak{X}_{r_{n}}^{H}\left(X_{1}, Z_{1} ; K\right)\right|>w \mid X_{1}=x, Z_{1}=z\right) .
\end{aligned}
$$

Proof. Let $\Gamma_{n}(x, z)=\cup_{\left(x^{\prime}, z^{\prime}\right) \in \mathfrak{X}_{r_{n}}^{M}(x, z ; K)} \mathfrak{X}_{r_{n}}^{D}\left(x^{\prime}, z^{\prime}\right)$, where conditional on particle types in $\mathfrak{X}_{r_{n}}^{M}(x, z ; K),\left\{\mathfrak{X}_{r_{n}}^{D}\left(x^{\prime}, z^{\prime}\right):\left(x^{\prime}, z^{\prime}\right) \in \mathfrak{X}_{r_{n}}^{M}(x, z ; K)\right\}$ are independent branching processes with intensity (A.3). Then $\mathfrak{X}_{r_{n}}^{H}(x, z ; K)=\cup_{\left(x^{\prime}, z^{\prime}\right) \in \Gamma_{n}(x, z)} \mathfrak{X}_{r_{n}}^{M}\left(x^{\prime}, z^{\prime} ; 1\right)$, where, conditional on $\Gamma_{n}(x, z),\left\{\mathfrak{X}_{r_{n}}^{M}\left(x^{\prime}, z^{\prime} ; 1\right):\left(x^{\prime}, z^{\prime}\right) \in \Gamma_{n}(x, z)\right\}$ are independent branching processes with intensity (A.5).

By the law of total probability,

$$
\begin{aligned}
\mathbf{P}\left(\left|\mathfrak{X}_{r_{n}}^{H}(x, z ; K)\right|>w\right) \leqslant \mathbf{P}\left(\left|\Gamma_{n}(x, z)\right|>w^{\prime}\right) & \\
& +\mathbf{P}\left(\sum_{\left(x^{\prime}, z^{\prime}\right) \in \Gamma_{n}(x, z)}\left|\mathfrak{X}_{r_{n}}^{M}\left(x^{\prime}, z^{\prime} ; 1\right)\right|>w \cap\left|\Gamma_{n}(x, z)\right| \leqslant w^{\prime}\right) .
\end{aligned}
$$




\section{LEUnG AND MoOn}

For any $\alpha>1$, the second term on the right-hand side equals

$$
\begin{array}{r}
\mathbf{E}\left[\mathbf{P}\left(\sum_{\left(x^{\prime}, z^{\prime}\right) \in \Gamma_{n}(x, z)}\left|\mathfrak{X}_{r_{n}}^{M}\left(x^{\prime \prime}, z^{\prime \prime} ; 1\right)\right|>w \mid \Gamma_{n}(x, z)\right) \mathbf{1}\left\{\left|\Gamma_{n}(x, z)\right| \leqslant w^{\prime}\right\}\right] \\
\leqslant \alpha^{-w} \mathbf{E}\left[\prod_{\left(x^{\prime}, z^{\prime}\right) \in \Gamma_{n}(x, z)} \mathbf{E}\left[\alpha^{\left|\mathfrak{X}_{r_{n}}^{M}\left(x^{\prime \prime}, z^{\prime \prime} ; 1\right)\right|} \mid \Gamma_{n}(x, z)\right] \mathbf{1}\left\{\left|\Gamma_{n}(x, z)\right| \leqslant w^{\prime}\right\}\right] \\
\leqslant \alpha^{-w}\left(\sup _{n>n_{0}} \sup _{\left(x^{\prime \prime}, z^{\prime \prime}\right) \in \mathcal{T}} \mathbf{E}\left[\alpha^{\left|\mathfrak{X}_{r_{n}}^{M}\left(x^{\prime \prime}, z^{\prime \prime} ; 1\right)\right|}\right]\right)^{w^{\prime}},
\end{array}
$$

where the second line uses Markov's inequality and conditional independence of the branching processes, and the third line also uses conditional independence.

By Lemma SA.2.2, we can choose $\alpha$ and $n_{0}$ such that the supremum term in (SA.2.5) is bounded above by some $C>0$. Then for any $c>0$ and $w^{\prime}=c w$,

$$
(\mathrm{SA} .2 .5)<\exp \{-w \log \alpha+c w \log C\} .
$$

Choosing $c$ such that $\log \alpha>c \log C$, the second element on the right-hand side of (SA.2.4) is bounded above by $e^{-\beta w}$ for some $\beta>0$. By a similar argument,

$$
\begin{aligned}
\mathbf{P}\left(\left|\Gamma_{n}(x, z)\right|>w^{\prime}\right) \leqslant \alpha^{-w^{\prime}}\left(\sup _{n>n_{0}} \sup _{\left(x^{\prime}, z^{\prime}\right) \in \mathcal{T}} \mathbf{E}\left[\alpha^{\left|\mathfrak{X}_{r_{n}}^{D}\left(x^{\prime}, z^{\prime}\right)\right|}\right]\right)^{w^{\prime \prime}} \\
\\
+\mathbf{P}\left(\mathfrak{X}_{r_{n}}^{M}(x, z ; K)>w^{\prime \prime}\right) .
\end{aligned}
$$

By Lemma SA.2.1, we can choose $\alpha, n_{0}$ such that the supremum term is bounded by a finite constant. Then choosing $w^{\prime \prime}=c^{\prime} w^{\prime}$ for some small enough $c^{\prime}>0$, the first term on the right-hand side can be bounded above by $e^{-\beta^{\prime} w^{\prime}}=e^{-\beta^{\prime} c w}$ for some $\beta^{\prime}>0$, recalling $w^{\prime}=c w$. By Lemma SA.2.2, the second term on the right-hand side of (SA.2.6) is $O\left(e^{-\beta^{\prime \prime} w^{\prime \prime}}\right)$ for some $\beta^{\prime \prime}>0$, uniformly over all $n$ sufficiently large. We have therefore established that (SA.2.4) is $O\left(e^{-\gamma w}\right)$ for some $\gamma>0$.

The last lemma bounds the maximal distance between the positions of $i$ and $\mathcal{N}_{\boldsymbol{J}(m+k)}\left(i, K, w^{\prime}\right)$ defined in (A.9).

Lemma SA.2.4. Under Assumptions 1, 7, and 9, for any $\epsilon>0, k \in\{1, \ldots, 4\}$, $k^{\prime} \in\{1,2\}$, and $K \in \mathbb{N}$, there exist constants $n_{0}, c>0$ such that for $w, w^{\prime} \rightarrow \infty$ with 
Normal Approximation in Large Network Models

$$
\begin{aligned}
& w / w^{\prime} \rightarrow \alpha \in(0, \infty), \\
& \max \left\{\sup _{n>n_{0}} \sup _{m \in((1-\epsilon) n,(1+\epsilon) n)} \sup _{\boldsymbol{x}_{k} \in \operatorname{supp}(f)^{k}} \mathbf{P}\left(\max _{i \in \mathcal{N}_{\boldsymbol{J}(m+k)}\left(1, K, w^{\prime}\right)} r_{n}^{-1}\left\|X_{1}-X_{i}\right\|>w \mid \boldsymbol{X}_{k}=\boldsymbol{x}_{k}\right),\right. \\
&\left.\sup _{n>n_{0}} \sup _{\boldsymbol{x}_{k^{\prime}} \in \operatorname{supp}(f)^{k^{\prime}}} \mathbf{P}\left(\max _{i \in \mathcal{N}_{\boldsymbol{J}\left(N_{n}+k^{\prime}\right)}\left(1, K, w^{\prime}\right)} r_{n}^{-1}\left\|X_{1}-X_{i}\right\|>w \mid \boldsymbol{X}_{k^{\prime}}=\boldsymbol{x}_{k^{\prime}}\right)\right\} \\
&=O\left(w^{d+1} \exp \{-c w\}\right) .
\end{aligned}
$$

Proof. We prove the bound for the probability involving $\mathcal{N}_{\boldsymbol{J}\left(N_{n}+k^{\prime}\right)}\left(1, K, w^{\prime}\right)$ for $k^{\prime}=1$. The argument for the other terms is very similar. Abbreviate $\boldsymbol{M}=\boldsymbol{M}\left(N_{n}+1\right)$ with $i j$ th entry $M_{i j}$ and $\boldsymbol{D}=\boldsymbol{D}\left(N_{n}+1\right)$ with $i j$ th entry $D_{i j}$. Let $\mathcal{N}_{\boldsymbol{J}\left(N_{n}+1\right)}^{\partial}\left(1, \ell, \ell^{\prime}\right)$ be the set of nodes at path distance $\ell^{\prime}$ from some node $j$ in the network $\boldsymbol{D}$ such that $j$ itself is path distance $\ell$ from node 1 in the network $\boldsymbol{M}$. Then

$$
\begin{aligned}
\mathbf{P}\left(\max _{i \in \mathcal{N}_{\boldsymbol{J}\left(N_{n}+1\right)}^{\left(1, K, w^{\prime}\right)}}\right. & \left.r_{n}^{-1}\left\|X_{1}-X_{i}\right\|>w\right) \\
& \leqslant \sum_{\ell=1}^{K} \mathbf{P}\left(\max _{i \in \mathcal{N}_{\boldsymbol{J}\left(N_{n}+1\right)}^{0}(1, \ell, 0)} r_{n}^{-1}\left\|X_{1}-X_{i}\right\|>w\right) \\
& +\sum_{\ell^{\prime}=0}^{w^{\prime}} \mathbf{P}\left(\max _{i \in \mathcal{N}_{\boldsymbol{J}\left(N_{n}+1\right)}^{0}\left(1,0, \ell^{\prime}\right)} r_{n}^{-1}\left\|X_{1}-X_{i}\right\|>w\right) \\
& +\sum_{\ell=1}^{K} \sum_{\ell^{\prime}=1}^{w^{\prime}} \mathbf{P}\left(\max _{i \in \mathcal{N}_{\boldsymbol{J}\left(N_{n}+1\right)}^{\partial}\left(1, \ell, \ell^{\prime}\right)} r_{n}^{-1}\left\|X_{1}-X_{i}\right\|>w\right) .
\end{aligned}
$$

We begin by bounding the third term on the right-hand side. Under the event that $\max _{i \in \mathcal{N}_{J\left(N_{n}+1\right)}^{\partial}\left(1, \ell, \ell^{\prime}\right)} r_{n}^{-1}\left\|X_{1}-X_{i}\right\|>w$, there must exist distinct nodes $j_{0} \neq \cdots \neq j_{\ell+\ell^{\prime}}$ with $j_{0}=1$ such that (a) for all $k=1, \ldots, \ell$, we have $M_{j_{k-1} j_{k}}=1$; (b) for all $k^{\prime}=\ell+1, \ldots, \ell+\ell^{\prime}$, we have $D_{j_{k^{\prime}-1} j_{k^{\prime}}}=1$; and (c) for some $m=1, \ldots, \ell+\ell^{\prime}$, we have $r_{n}^{-1}\left\|X_{j_{m-1}}-X_{j_{m}}\right\|>w /\left(\ell+\ell^{\prime}\right)$. By Assumption 1 , since $D_{i j} \leqslant M_{i j}$ for all $i, j$, we have $\zeta_{j_{m-1} j_{m}}>\bar{V}^{-1}\left(w /\left(\ell+\ell^{\prime}\right), 0\right)$ for $w$ sufficiently large and the $m$ in part (c). 


\section{LEUNG AND MOON}

Using these facts,

$$
\begin{aligned}
& \mathbf{P}\left(\max _{i \in \mathcal{N}_{\boldsymbol{J}\left(N_{n}+1\right)}^{\partial}\left(1, \ell, \ell^{\prime}\right)} r_{n}^{-1}\left\|X_{1}-X_{i}\right\|>w\right) \\
& \leqslant \mathbf{P}\left(\bigcup_{j_{0} \neq \cdots \neq j_{\ell+\ell^{\prime}} \in \mathcal{N}_{N_{n}+1}, j_{0}=1} \bigcup_{m=1}^{\ell+\ell^{\prime}}\left\{\zeta_{j_{m-1} j_{m}}>\bar{V}^{-1}\left(w /\left(\ell+\ell^{\prime}\right), 0\right)\right\}\right. \\
& \left.\cap\left\{\prod_{k=1}^{\ell} \prod_{k^{\prime}=\ell+1}^{\ell+\ell^{\prime}} M_{j_{k^{\prime}-1} j_{k^{\prime}}} D_{j_{k^{\prime}-1} j_{k^{\prime}}}=1\right\}\right) \\
& \leqslant \mathbf{E}\left[\sum_{j_{0} \neq \cdots \neq j_{\ell} \in \mathcal{N}_{N_{n}+1}, j_{0}=1} \sum_{m=1}^{\ell+\ell^{\prime}} \rho_{n}\left(X_{j_{m-1}}, X_{j_{m}}, w /\left(\ell+\ell^{\prime}\right)\right)\right. \\
& \left.\times \prod_{\substack{k=1 \\
k \neq m}}^{\ell} \bar{p}_{r_{n}}\left(X_{j_{k-1}}, X_{j_{k}}\right) \prod_{\substack{k^{\prime}=\ell+1 \\
k^{\prime} \neq m}}^{\ell+\ell^{\prime}} p_{r_{n}}\left(X_{j_{k^{\prime}-1}}, Z_{j_{k^{\prime}-1}}, X_{j_{k^{\prime}}}, Z_{j_{k^{\prime}}}\right)\right]
\end{aligned}
$$

where $\bar{p}_{r_{n}}(\cdot)$ is defined in (A.4), $p_{r_{n}}(\cdot)$ is defined in (15), and

$$
\begin{aligned}
& \rho_{n}\left(X_{j_{m-1}}, X_{j_{m}}, w /\left(\ell+\ell^{\prime}\right)\right) \equiv \\
& \quad \mathbf{P}\left(\zeta_{j_{m-1} j_{m}}>\max \left\{\bar{V}^{-1}\left(w /\left(\ell+\ell^{\prime}\right), 0\right), \bar{V}^{-1}\left(r_{n}^{-1}\left\|X_{j_{m-1}}-X_{j_{m}}\right\|, 0\right)\right\} \mid X_{j_{m-1}}, X_{j_{m}}\right) .
\end{aligned}
$$

By Corollary 3.2.3 of Schneider and Weil (2008), the last line is bounded above by

$$
\begin{aligned}
&\left(\ell+\ell^{\prime}\right) \int_{\mathbb{R}^{d}} \cdots \int_{\mathbb{R}^{d}} \prod_{k=1}^{\ell} \bar{p}_{r_{n}}\left(x_{j_{k-1}}, x_{j_{k}}\right) n f\left(x_{j_{k-1}}\right) \mathrm{d} x_{j_{k-1}} \\
& \times \mathbf{E}\left[\prod_{k^{\prime}=\ell+1}^{\ell+\ell^{\prime}-1} p_{r_{n}}\left(x_{j_{k^{\prime}-1}}, Z_{j_{k^{\prime}-1}}, x_{j_{k^{\prime}}}, Z_{j_{k^{\prime}}}\right) \mid \mathcal{F}\right] \prod_{k^{\prime}=\ell+1}^{\ell+\ell^{\prime}-1} n f\left(x_{j_{k^{\prime}-1}}\right) \mathrm{d} x_{j_{k^{\prime}-1}} \\
& \quad \times \rho_{n}\left(x_{j_{\ell+\ell^{\prime}-1}}, x_{\ell+\ell^{\prime}}, w /\left(\ell+\ell^{\prime}\right)\right) n f\left(x_{\ell+\ell^{\prime}-1}\right) \mathrm{d} x_{\ell+\ell^{\prime}-1} f\left(x_{\ell+\ell^{\prime}}\right) \mathrm{d} x_{\ell+\ell^{\prime}},
\end{aligned}
$$

where $\mathcal{F}=\left\{X_{j_{\ell}}=x_{j_{\ell}}, \ldots, X_{j_{\ell+\ell-1}}=x_{j_{\ell+\ell^{\prime}-1}}\right\}$. This is bounded above by

$$
\left(\ell+\ell^{\prime}\right)\left(\sup _{x} n \int_{\mathbb{R}^{d}} \bar{p}_{r_{n}}(x, y) f(y) \mathrm{d} y\right)^{\ell}\left(\left\|h_{\boldsymbol{D}}\right\|_{\mathbf{m}}\right)^{\ell^{\prime}-1} n \mathbf{E}\left[\rho_{n}\left(X_{1}, X_{2}, w /\left(\ell+\ell^{\prime}\right)\right)\right]
$$

where $\left\|h_{\boldsymbol{D}}\right\|_{\mathbf{m}}<1$ by Assumption 7. Then using Assumption 1, there exist $c_{1}, c_{2}>0$ 


\section{Normal Approximation in Large Network Models}

such that this is bounded above by

$$
\begin{aligned}
\left(\ell+\ell^{\prime}\right) \cdot c_{1}^{\ell} \cdot c_{2}^{-\ell^{\prime}+1} \cdot \kappa \bar{f}\left(\int_{w /\left(\ell+\ell^{\prime}\right)}^{\infty} u^{d-1} \bar{\Phi}_{\zeta}\left(\bar{V}^{-1}(u, 0)\right) \mathrm{d} u\right. & \\
& \left.+\frac{\left(w /\left(\ell+\ell^{\prime}\right)\right)^{d}}{d} \bar{\Phi}_{\zeta}\left(\bar{V}^{-1}\left(w /\left(\ell+\ell^{\prime}\right), 0\right)\right)\right),
\end{aligned}
$$

by a change of variables to polar coordinates. Since $\bar{\Phi}_{\zeta}\left(\bar{V}^{-1}(u, 0)\right) \leqslant c_{3} e^{-c_{4} u}$ for some $c_{3}, c_{4}>0$ by Assumption 1, the second term in the parentheses is at least as large as the first term in an asymptotic sense for $w$ large, as can be seen by integration by parts. Then the previous expression is bounded above by

$$
\begin{aligned}
\left(\ell+\ell^{\prime}\right) \cdot c_{1}^{\ell} \cdot c_{2}^{-\ell^{\prime}+1} \cdot \kappa \bar{f}\left(w /\left(\ell+\ell^{\prime}\right)\right)^{d} c_{3} \exp \left\{-c_{4} w /\left(\ell+\ell^{\prime}\right)\right\} & \\
& <C\left(\ell+\ell^{\prime}\right)\left(w /\left(\ell+\ell^{\prime}\right)\right)^{d} \exp \left\{-c\left(w /\left(\ell+\ell^{\prime}\right)-\ell+\ell^{\prime}-1\right)\right\}
\end{aligned}
$$

for $w$ sufficiently large and some $C, c>0$ that do not depend on $w, \ell, \ell^{\prime}$.

Therefore, the third term on the right-hand side of (SA.2.7) is bounded above by

$$
\begin{aligned}
& C \sum_{\ell=1}^{K} \sum_{\ell^{\prime}=1}^{w^{\prime}}\left(\ell+\ell^{\prime}\right)\left(w /\left(\ell+\ell^{\prime}\right)\right)^{d} \exp \left\{-c\left(w /\left(\ell+\ell^{\prime}\right)-\ell+\ell^{\prime}-1\right)\right\} \\
& \quad<C K\left(w^{\prime}+K\right) w^{d} \sum_{\ell^{\prime}=1}^{w^{\prime}} \exp \left\{-c\left(w /\left(K+\ell^{\prime}\right)+\ell^{\prime}-K-1\right)\right\} \\
& \leqslant C^{\prime} w^{\prime} w^{d}\left(\int_{0}^{w^{\prime}} \exp \left\{-c\left(w /\left(K+\ell^{\prime}\right)+\ell^{\prime}\right)\right\} \mathrm{d} \ell^{\prime}-e^{-c(w / K)}+e^{-c\left(w /\left(w^{\prime}+K\right)+w^{\prime}\right)}\right) .
\end{aligned}
$$

for some $C^{\prime}>0$ that depends on $K$. For $w$ sufficiently large, the integral is bounded above by

$$
\begin{aligned}
\int_{0}^{w^{\prime}}\left(\frac{w}{\left(K+\ell^{\prime}\right)^{2}}-1\right) \exp & \left\{-c\left(\frac{w}{K+\ell^{\prime}}+\ell^{\prime}\right)\right\} \mathrm{d} \ell^{\prime} \\
& =-\frac{1}{c}\left(\exp \left\{-c\left(w /\left(K+w^{\prime}\right)+w^{\prime}\right)\right\}-\exp \{-c w / K\}\right) .
\end{aligned}
$$

Hence, $(\mathrm{SA} .2 .8)=O\left(w^{d+1} \exp \{-c w\}\right)$ for some $c>0$. By a similar argument, the other terms on the right-hand side of (SA.2.7) are also of this order. 